\title{
4. PETROLOGY OF BASALTS FROM HOLE 504B, DEEP SEA DRILLING PROJECT, LEG $8^{1}$
}

\author{
P. D. Kempton, L. K. Autio, J. M. Rhodes, M. J. Holdaway, M. A. Dungan, and P. Johnson²
}

\begin{abstract}
Basalts from Hole 504B, Leg 83, exhibit remarkable uniformity in major and trace element composition throughout the $1075.5 \mathrm{~m}$ of basement drilled. The majority of the basalts, Group $\mathrm{D}^{\prime}$, have unusual compositions relative to normal (Type I) mid-ocean ridge basalts (MORB). These basalts have relatively high $\mathrm{mg}$ values $(0.60-0.70)$ and $\mathrm{CaO}$ abundances $(11.7-13.7 \% ; \mathrm{Ca} / \mathrm{Al}=0.78-0.89)$, but exhibit a marked depletion in compatible trace elements $(\mathrm{Cr}$ and $\mathrm{Ni})$; moderately incompatible trace elements ( $\mathrm{Zr}, \mathrm{Y}, \mathrm{Ti}$, etc.); and highly incompatible trace elements (Nb, LREE, etc.). Petrographic and compositional data indicate that most of these basalts are evolved, having fractionated significant amounts of plagioclase, olivine, and clinopyroxene. Melting experiments on similar basalt compositions from the upper portion of Hole 504B (Leg 70; Autio and Rhodes, 1983) indicate that the basalts are co-saturated with olivine and plagioclase and often clinopyroxene on the 1-atm. liquidus.

Two rarely occurring groups, $\mathbf{M}^{\prime}$ and $\mathrm{T}$, are compositionally distinct from Group $\mathrm{D}^{\prime}$ basalts. Group $\mathrm{T}$ is strongly depleted in all magmaphile elements except the highly incompatible ones ( $\mathrm{Nb}, \mathrm{La}$, etc.), while Group $\mathrm{M}^{\prime}$ has moderate concentrations of both moderately and highly incompatible trace elements and is similar to Type I MORB. Groups M' and $\mathrm{T}$ cannot be related to Group $\mathrm{D}^{\prime}$ nor to each other by crystal fractionation, crystal accumulation, or magma mixing. The large differences in magmaphile element ratios $(\mathrm{Zr} / \mathrm{Nb}, \mathrm{La} / \mathrm{Yb})$ among these three chemical groups may be accounted for by complex melting models and/or local heterogeneity of the mantle beneath the Costa Rica Ridge.

Xenocrysts and xenoliths of plagioclase and clinopyroxene similar in texture and mineral composition to crystals in coarse-grained basalts from the lower portion of the hole are common in Hole 504B basalts. These suggest that addition of solid components either from conduit or magma chamber walls has occurred and may be a common source of disequilibrium crystals in these basalts. However, mixing of plagioclase-laden depleted melts (similar to the Costa Rica Ridge Zone basalts) with normal MORB magmas could provide an alternate source for some refractory plagioclase crystals found out of equilibrium in many phyric MORB.

The uniformity of major element compositions in Hole 504B basalts affords an ideal situation for investigating the effects of alteration on some major and trace elements in oceanic basalts. Alteration observed in whole-rock samples records primarily two events-a high-temperature and a low-temperature phase. High-temperature phases include: chlorite, talc, albite, actinolite, sphene, quartz, and pyrite. The low-temperature phases include smectite (saponite), epistilbite or laumontite, and minor calcite. Laumontite may actually straddle the gap between the low- and high-temperature mineral assemblages. Alteration is restricted primarily to partial replacement of primary phases. Metamorphic grade, in general, increases from the top to the bottom of Hole 504B (Legs 69, 70, and 83) as seen in the change from a smectiteto-chlorite-dominated secondary mineral assemblage. However, a systematic progression for the interval recovered during Leg 83 is not apparent. Rather, the extent of alteration appears to be a function of the initial texture and fracture density. Variations in whole-rock major and trace element concentrations cannot be attributed convincingly to any differences in alteration observed. Compositional characteristics of the secondary minerals indicated that extensive remobilization of elements has not occurred; local redistribution is suggested in most cases. Thus, the major and trace element signature of these basalts remains effectively the same as the original composition prior to alteration.
\end{abstract}

\section{INTRODUCTION}

Basalt samples from Hole 504B (DSDP Legs 69, 70, and 83 ) provide a unique opportunity for sampling the upper part of the oceanic crust. Leg 83 has extended Hole 504B by $514 \mathrm{~m}$ to a total depth of $1350 \mathrm{~m}$ below the seafloor (BSF). Of this total, $1075.5 \mathrm{~m}$ were basement. This is the deepest penetration of the oceanic basement to date. The upper portion of Hole 504B (Legs 69 and 70) consists mainly of pillow lavas and massive flows (Layer 2A). An increasing proportion of dikes were encountered during Leg 83, with few flows or pillows occurring below $1055 \mathrm{~m}$ BSF. Leg 83 represents the first unequivocal sampling of oceanic Layer $2 \mathrm{C}$, the sheeted

\footnotetext{
${ }^{1}$ Anderson, R. N., Honnorez, J., Becker, K., et al., Init. Repts. DSDP, 83: Washington (U.S., Govt. Printing Office).

2 Addresses: (Kempton) NASA-Johnson Space Center, Mail Code SN2, Houston, TX 77058; (Holdaway, Dungan, Johnson) Department of Geological Sciences, Southern Methodist University, Dallas, TX 75275; (Autio, Rhodes) Department of Geology and Geography, University of Massachusetts, Amherst, MA 01003.
}

dike complex. Further details of basement penetration and recovery are given in the site chapter (this volume).

Mineral chemistry and petrography, whole-rock chemistry, and alteration effects in Leg 83 basalts are examined in this chapter. These data are compared to Leg 69 and 70 data for the upper part of Hole 504B (e.g., Autio and Rhodes, 1983; Natland et al., 1983) to determine the chemical and petrological relationship of Layers 2A, B, and C. Data from the Costa Rica Rift Zone (CRRZ) are also compared to other mid-ocean ridge basalt (MORB) data. Extensive whole-rock data are used to demonstrate the unusual chemical characteristics of the CRRZ basalts. Mineral chemistry and zoning and bulk rock chemistry are used to evaluate magmatic processes associated with formation of oceanic crust at the Costa Rica Rift and to place chemical and mineralogical constraints on the mantle source.

The CRRZ basalts vary from slightly olivine to slightly quartz-normative tholeiites. The samples studied range from aphyric to sparsely phyric. Plagioclase is the most common phenocryst phase followed by olivine, clinopy- 
roxene, and spinel. Representative microprobe analyses are given in Tables 1-4. Samples were chosen to reflect minimal alteration effects and do not include brecciated or veined portions. Whole-rock chemistry is given in Tables 5-8.

\section{EXPERIMENTAL METHODS}

Thin sections were cut from cores and studied petrographically at Southern Methodist University (SMU). Microprobe analyses were performed on a JEOL 733 electron microprobe at SMU, using mineral standards at $15-\mathrm{kV}$ accelerating potential, a $20-\mathrm{nA}$ sample current, and three wavelength dispersive spectrometers. Raw data were processed on a PDP-11 computer to correct for dead time, background and absorption, fluorescence, and atomic number (ZAF) using the alpha correction for oxides developed by Bence and Albee (1968). Backscattered electron imaging (BEI) was performed on the same instrument at low sample currents (2-4 nA) using standard polished thin sections to map mineralogical and textural variations that were too fine to resolve petrographically.

Core samples $(\sim 25 \mathrm{~g})$ were crushed in a tungsten carbide shatterbox at SMU and split into aliquots for X-ray fluorescence (XRF) and flame photometric analysis at the University of Massachusetts and instrumental neutron activation analysis (INAA) at the Johnson Space Center in Houston, Texas. INAA was used to determine rare earth element (REE: $\mathrm{La}, \mathrm{Ce}, \mathrm{Nd}, \mathrm{Sm}, \mathrm{Eu}, \mathrm{Tb}, \mathrm{Yb}$, and $\mathrm{Lu}$ ), trace element (Co, $\mathrm{Hf}, \mathrm{Ta}$, and $\mathrm{Sc}$ ), and $\mathrm{Na}_{2} \mathrm{O}$ abundances for 16 samples. Twenty-milligram aliquots were sealed into glass tubes and irradiated in the rotisserie can of the modified Triga Mark III reactor of the Nuclear Science Center of Texas A\&M University. Samples were irradiated for 14-hour periods on two successive days, receiving a flux of $6-8 \times 10^{12}$ neutrons $/ \mathrm{cm}$ per $\mathrm{s}$. Following irradiation, three measurements ranging from 2 to 8 hours in length were made using a coaxial $\mathrm{Ge}(\mathrm{Li})$ detector during a time period of 7-40 days. USGS standards BCR, BHVO, and
DTS- 1 were used as calibration standards. Data were reduced using GELIGAM EG \& G ORTEC software (version Gamma 2).

An automated Siemens SRS-2 XRF spectrometer was used to determine bulk rock chemistries. Powders for major element XRF analyses were dried and oxidized in air at $1000^{\circ} \mathrm{C}$ for $1 \mathrm{hr}$. All major elements except $\mathrm{Na}$ were determined by XRF on La-bearing, lithium borate fused glass disks (Norrish and Hutton, 1969) using a chromium tube. $\mathrm{Na}$ values were determined by INAA or flame photometry using an IL 443 flame photometer. The flame photometric determination of $\mathrm{Na}$ used $\mathrm{Li}$ as an internal standard, with USGS standards AGV-1 and PCC-1 used for calibration. The method followed is that of Bernas (1968) modified by K. Hollocher (personal communication, 1982). INAA and flame-photometric values agree within $\pm 0.05 \mathrm{wt} . \%$.

Trace elements were determined on unfired pressed powder pellets by $\mathrm{XRF}$ analysis (Y, $\mathrm{Sr}, \mathrm{Rb}$, and $\mathrm{Ga}$ with an $\mathrm{Mo}$ tube; $\mathrm{Zr}, \mathrm{Nb}, \mathrm{Zn}, \mathrm{Ni}$, $\mathrm{Cr}$, and $\mathrm{V}$ with an Au tube) using a modification of the method of Norrish and Chappell (1967). Calibrations were based on both synthetic mixtures and well-analyzed rock standards. Corrections were made for tube contamination, interelement interference, and background nonlinearity. A Compton scattering method, modified from that of Reynolds (1967), was used to correct for differences in mass absorption coefficients between samples and standards.

Average values for BCR-1 analyzed as an unknown by XRF are given in Table 5, with one standard deviation. Each element was analyzed twice in each sample, except for $\mathrm{Nb}$ which was measured six times in order to improve sensitivity at the low concentration levels involved $(<0.5-2 \mathrm{ppm})$. Error values labeled CRRZ in Table 5 are based on these replicate analyses and apply directly to the CRRZ basalts under the given operating conditions. The values were calculated using the formula:

$$
s=\sqrt{\left(x-\bar{x}^{-2} /(n-1)\right.}
$$

where $x$ and $\overline{\mathrm{x}}$ are observation and mean for replicate analyses of the element in a single sample and $n$ is the number of samples analyzed.

Table 1. Microprobe analyses of clinopyroxenes, Leg 83.

\begin{tabular}{|c|c|c|c|c|c|c|c|c|c|c|c|}
\hline & $\begin{array}{c}(1) \\
83-4^{a}\end{array}$ & $\begin{array}{c}(2) \\
83-4\end{array}$ & $\begin{array}{c}(3) \\
83-4\end{array}$ & $\begin{array}{c}(4) \\
83-13\end{array}$ & $\begin{array}{c}(5) \\
83-13\end{array}$ & $\begin{array}{c}(6) \\
83-22\end{array}$ & $\begin{array}{c}(7) \\
83-22\end{array}$ & $\begin{array}{c}(8) \\
83-22\end{array}$ & $\begin{array}{c}(9) \\
83-45\end{array}$ & $\begin{array}{c}(10) \\
83-52\end{array}$ & $\begin{array}{c}(11) \\
83-52\end{array}$ \\
\hline $\mathrm{SiO}_{2}$ & 52.87 & 51.62 & 49.96 & 51.97 & 50.48 & 49.77 & 46.67 & 49.46 & 51.48 & 52.03 & 50.85 \\
\hline $\mathrm{TiO}_{2}$ & 0.23 & 0.75 & 0.90 & 0.34 & 0.90 & 1.45 & 2.59 & 1.17 & 0.54 & 0.25 & 0.68 \\
\hline $\mathrm{Al}_{2} \mathrm{O}_{3}$ & 2.73 & 3.83 & 1.12 & 3.20 & 2.61 & 4.38 & 6.88 & 2.29 & 2.08 & 3.27 & 1.17 \\
\hline $\mathrm{Cr}_{2} \mathrm{O}_{3}$ & 0.63 & 0.39 & 0.13 & 1.41 & 0.02 & 0.12 & 0.31 & 0.02 & 0.04 & 1.21 & 0.00 \\
\hline $\mathrm{FeO}$ & 4.36 & 6.77 & 22.35 & 4.14 & 13.71 & 8.09 & 8.89 & 15.37 & 13.22 & 4.04 & 20.00 \\
\hline $\mathrm{MnO}$ & 0.13 & 0.20 & 0.64 & 0.09 & 0.38 & 0.23 & 0.28 & 0.53 & 0.41 & 0.15 & 0.68 \\
\hline $\mathrm{MgO}$ & 18.39 & 17.07 & 12.37 & 17.75 & 14.72 & 14.33 & 13.10 & 13.13 & 16.60 & 17.99 & 12.55 \\
\hline $\mathrm{CaO}$ & 20.20 & 19.16 & 12.47 & 21.13 & 17.64 & 22.08 & 21.14 & 17.50 & 15.51 & 21.05 & 14.71 \\
\hline $\mathrm{Na}_{2} \mathrm{O}$ & 0.20 & 0.21 & 0.17 & 0.23 & 0.28 & 0.35 & 0.39 & 0.36 & 0.23 & 0.25 & 0.16 \\
\hline Total & 99.73 & 99.99 & 100.11 & 100.27 & 100.75 & 100.80 & 100.26 & 99.84 & 100.11 & 100.24 & 100.81 \\
\hline
\end{tabular}

\begin{tabular}{|c|c|c|c|c|c|c|c|c|c|c|c|}
\hline $\mathrm{Si}$ & 1.927 & 1.892 & 1.938 & 1.894 & 1.895 & 1.844 & 1.750 & 1.892 & 1.923 & 1.894 & 1.944 \\
\hline $\mathrm{Ti}$ & 0.005 & 0.020 & 0.026 & 0.008 & 0.025 & 0.040 & 0.072 & 0.033 & 0.014 & 0.006 & 0.019 \\
\hline $\mathrm{Al}$ & 0.116 & 0.164 & 0.050 & 0.137 & 0.114 & 0.191 & 0.304 & 0.103 & 0.091 & 0.140 & 0.053 \\
\hline $\mathrm{Cr}$ & 0.017 & 0.011 & 0.003 & 0.041 & 0.000 & 0.003 & 0.009 & 0.000 & 0.001 & 0.034 & 0.000 \\
\hline $\mathrm{Fe}$ & 0.132 & 0.206 & 0.724 & 0.126 & 0.430 & 0.250 & 0.278 & 0.492 & 0.412 & 0.123 & 0.638 \\
\hline $\mathrm{Mn}$ & 0.003 & 0.005 & 0.020 & 0.002 & 0.011 & 0.006 & 0.009 & 0.017 & 0.012 & 0.004 & 0.021 \\
\hline $\mathrm{Mg}$ & 0.999 & 0.932 & 0.715 & 0.963 & 0.823 & 0.791 & 0.732 & 0.749 & 0.924 & 0.976 & 0.714 \\
\hline $\mathrm{Ca}$ & 0.788 & 0.751 & 0.518 & 0.824 & 0.709 & 0.876 & 0.849 & 0.717 & 0.621 & 0.820 & 0.603 \\
\hline $\mathrm{Na}$ & 0.013 & 0.014 & 0.012 & 0.016 & 0.020 & 0.023 & 0.028 & 0.027 & 0.016 & 0.017 & 0.011 \\
\hline Total & 4.000 & 3.995 & 4.006 & 4.010 & 4.026 & 4.024 & 4.031 & 4.029 & 4.014 & 4.014 & 4.003 \\
\hline Wo & 0.414 & 0.401 & 0.269 & 0.438 & 0.374 & 0.472 & 0.475 & 0.381 & 0.326 & 0.437 & 0.313 \\
\hline En & 0.525 & 0.498 & 0.371 & 0.512 & 0.434 & 0.426 & 0.409 & 0.397 & 0.484 & 0.520 & 0.371 \\
\hline Fs & 0.061 & 0.102 & 0.360 & 0.049 & 0.192 & 0.102 & 0.116 & 0.222 & 0.190 & 0.043 & 0.315 \\
\hline
\end{tabular}

Note: Numbers that follow are core-section, cm ranges of samples from Hole 504B. (1) 73-1, 126-130 cm: core of partially resorbed clinopyroxene in xenolith; (2) 73-1, 126-130 cm: core of groundmass grain; (3) 73-1, 126-130 cm: rim of groundmass clinopyroxene 2 ; (4) 88-1, 109-113 cm: core of large phenocryst with subophitic rim; (5) 88-1, 109-113 cm: rim of groundmass grain; (6) 93-3, 27-32 cm: core of subophitic groundmass grain; (7) 93-3, 27-32 cm: intermediate zone, maximum $\mathrm{TiO}_{2}$ concentration in groundmass clinopyroxene $6 ;(8) 93-3,27-32 \mathrm{~cm}$ : rim of groundmass clinopyroxene 6 adjacent to mesostasis. Notice decrease in $\mathrm{TiO}_{2}$ away from intermediate zone, analysis 7; (9) 123-1, 60-63 cm: rim of groundmass grain; (10) 129-1, $76-81 \mathrm{~cm}$ : Core of large subophitic grain; (11) 129-1, 76-81 cm: rim of groundmass clinopyroxene.

a Sample identification number. 
Table 2. Microprobe analyses of FeTi oxides, Leg 83.

\begin{tabular}{lrrrrrrr}
\hline & $(1)$ & \multicolumn{1}{c}{$(2)$} & \multicolumn{1}{c}{$(3)$} & \multicolumn{1}{c}{$(4)$} & \multicolumn{1}{c}{$(5)$} & \multicolumn{1}{c}{$(6)$} & \multicolumn{1}{c}{$(7)$} \\
& $83-4$ & $83-4$ & $83-32$ & $83-52$ & $83-22$ & $83-22$ & $83-52$ \\
\hline $\mathrm{SiO}_{2}$ & 0.14 & 0.14 & 0.13 & 0.15 & 0.42 & 0.40 & 0.30 \\
$\mathrm{TiO}_{2}$ & 19.46 & 19.76 & 22.14 & 21.22 & 47.70 & 48.08 & 48.73 \\
$\mathrm{Al}_{2} \mathrm{O}_{3}$ & 2.49 & 1.86 & 2.31 & 1.45 & 0.11 & 0.13 & 0.05 \\
$\mathrm{Cr}_{2} \mathrm{O}_{3}$ & 0.12 & 0.21 & 0.16 & 0.03 & 0.11 & 0.11 & 0.00 \\
$\mathrm{Fe}_{2} \mathrm{O}_{3} *$ & 28.10 & 27.62 & 23.48 & 26.86 & 12.85 & 12.6 & 8.82 \\
$\mathrm{FeO}$ & 46.73 & 46.30 & 49.01 & 47.62 & 32.80 & 33.12 & 38.46 \\
$\mathrm{MnO}$ & 1.30 & 2.38 & 1.34 & 1.65 & 5.19 & 4.97 & 3.09 \\
$\mathrm{MgO}$ & 0.08 & 0.05 & 0.11 & 0.11 & 0.09 & 0.07 & 0.19 \\
$\mathrm{CaO}$ & 9.14 & - & 0.21 & 0.24 & 0.85 & 0.97 & 0.27 \\
Total & 98.56 & 98.34 & 98.87 & 99.33 & 100.12 & 100.46 & 99.90
\end{tabular}

Cations calculated on the basis of 32 oxygens for $1-4 ; 6$ for $5-7$

\begin{tabular}{lrrrrrrr}
$\mathrm{Si}$ & 0.039 & 0.040 & 0.034 & 0.044 & 0.021 & 0.020 & 0.015 \\
$\mathrm{Ti}$ & 4.429 & 4.522 & 5.014 & 4.812 & 1.817 & 1.825 & 1.858 \\
$\mathrm{Al}$ & 0.886 & 0.666 & 0.818 & 0.514 & 0.006 & 0.008 & 0.003 \\
$\mathrm{Cr}$ & 0.029 & 0.050 & 0.038 & 0.001 & 0.004 & 0.004 & 0.000 \\
$\mathrm{Fe}+3$ & 6.392 & 6.318 & 5.315 & 6.086 & 0.489 & 0.479 & 0.336 \\
$\mathrm{Fe}$ & 11.812 & 11.769 & 12.324 & 11.993 & 1.387 & 1.396 & 1.628 \\
$\mathrm{Mn}$ & 0.333 & 0.613 & 0.341 & 0.421 & 0.222 & 0.212 & 0.133 \\
$\mathrm{Mg}$ & 0.036 & 0.023 & 0.049 & 0.049 & 0.007 & 0.005 & 0.014 \\
$\mathrm{Ca}$ & 0.049 & - & 0.061 & 0.070 & 0.047 & 0.053 & 0.014 \\
Total & 24.005 & 24.000 & 23.995 & 23.998 & 4.001 & 4.002 & 4.001 \\
\hline
\end{tabular}

Note: $\mathrm{Fe}_{2} \mathrm{O}_{3}{ }^{*}$ calculated assuming stoichiometry and charge balance. Numbers that follow are core-section, centimeter ranges of samples from Hole 504B. (1) $73-1,126-130 \mathrm{~cm}$ : unaltered subhedral magnetite; (2) 73-1, 126-130 cm: unaltered skeletal magnetite; (3) 104-2, 24-27 cm: partially altered magnetite; (4) 129-1, 76-81 cm: unaltered skeletal magnetite; (5) 93-3, 27-32 cm: partially altered primary ilmenite; (6) 93-3, 27-32 cm: secondary ilmenite in altered magnetite; (7) $129-1,76-81 \mathrm{~cm}$ : secondary ilmentite in altered interstitial area.

a Sample identification number.

REE and trace element values for BCR-1 analyzed as an unknown by INAA are given in Table 7 with one standard deviation. The calculated percent counting uncertainty $\left(\% U_{c} ; 1 \sigma\right)$ is calculated using the formula

$\frac{100 \times \sqrt{\text { gross area counts } \div \text { background count }}}{\text { net area counts }}$

net area counts

where background counts equal the summation of continuum, peaked background, and all net areas that are contained under the peak envelope other than the net peak being considered; $\mathrm{mg}$ values were calculated as molecular $\mathrm{Mg} /\left(\mathrm{Mg}+\mathrm{Fe}^{2+}\right)$, where molecular $\mathrm{Fe}$ has been proportioned as $\mathrm{Fe}^{3+} /\left(\mathrm{Fe}^{2+}+\mathrm{Fe}^{3+}\right)=0.1$ (Basaltic Volcanism Study Project, 1981).

\section{PETROGRAPHY AND MINERAL CHEMISTRY}

Basalts recovered from Hole 504B occur as pillows, dikes, and massive units. The massive units are those for which the mode of emplacement is uncertain; they are presumed to be flows or the interiors of large dikes. Hole 504B basalts can be divided into two major petrographic groups: aphyric and phyric basalts. The phyric basalts are further subdivided on the basis of the phenocryst assemblage; the relative abundance of each phase is not considered. The groups are labeled according to the first letter of each occurring phenocryst mineral: OPC-olivine, plagioclase, clinopyroxene phyric basalt; $\mathrm{OP}-$ olivine and plagioclase phyric basalt; and $\mathrm{A}-$ aphyric basalt. A similar classification exists for basalts described from Holes 501, 504A, and the upper $562 \mathrm{~m}$ of 504B (Natland et al., 1983). The classification proposed by Natland defines an additional group on the basis of chrome spinel present in OP basalts. Chrome spinel, however, was identified in each of the petrographic groups listed previously. Since no other petrographic or chemical characteristics were observed that discriminated among the $\mathrm{Cr}$ spinel-bearing samples, such a group was not included in the classification for Leg 83 basalts.

Estimated relative abundances of each petrographic group in the stratigraphic column support the conclusion derived on chemical grounds that evolved or multiply-saturated compositions predominate in Hole 504B. These estimates $(\mathrm{A}=42 \%, \mathrm{OPC}=37 \%$, and $\mathrm{OP}=$ $21 \%$ of the lower $514 \mathrm{~m}$ ) are based on the number of meters of core assigned to each lithologic unit defined by the shipboard party. It assumes that the petrographic group assigned to that lithologic unit remains unchanged throughout the unit.

\section{Aphyric Basalts}

The aphyric basalts contain less than $1 \%$ crystals that qualify as phenocrysts. They are, however, among the coarsest in grain size of the lavas from the lower portion of Hole 504B (Fig. 1). They have been recovered only from basalts identified as dikes or massive units. Groundmass textures are typically subophitic to intergranular. Grain sizes range from fine to medium.

Subhedral plagioclase laths constitute 35 to $60 \%$ (visual estimate) of the rock. The plagioclase ranges in size from 0.4 to $1.6 \mathrm{~mm}$ and typically forms radiate aggregates of two to four crystals. Although phenocrysts are not present, rare glomerocrystic clusters of larger, more equant plagioclase xenocrysts occur. In addition, some of the large plagioclase crystals have sievelike or porous textures that have been interpreted as the product of partial resorption (Dungan and Rhodes, 1978; Kuo and Kirkpatrick, 1982) and reproduced experimentally by Lofgren and Norris (1981). These will be discussed further in conjunction with the plagioclase phenocrysts and xenocrysts from the phyric basalts. In the aphyric units plagioclase composition ranges from $\mathrm{An}_{40}$ to $\mathrm{An}_{85}$ (Fig. 2).

Clinopyroxene constitutes 30 to $50 \%$ of the aphyric lavas. It occurs as equant, anhedral crystals in subophitic to intergranular textures or as plumose quench morphologies in rapidly cooled lavas. In samples with medium-grained subophitic/ophitic textures it forms anhedral crystals up to $2.5 \mathrm{~mm}$ in size. Rare clinopyroxene xenocrysts occur that have porous textures like those of the resorbed plagioclases (Fig. 3). The rims of these clinopyroxenes typically enclose groundmass plagioclase subophitically, but euhedral crystals are also present. Both exhibit twinning on (100). These xenocrystic clinopyroxenes are characteristically more $\mathrm{Mg}$ - and $\mathrm{Cr}$-rich than the coexisting groundmass clinopyroxenes (Fig. 4A and Table 1). The groundmass pyroxenes are also more strongly zoned; $\mathrm{FeO}$ increases from 6.93 to $18.61 \mathrm{wt} . \%$ at the expense of calcium and magnesium. They do not exhibit the enrichment in $\mathrm{TiO}_{2}$ observed in many other MORB clinopyroxenes (Dungan and Rhodes, 1978; Ayuso et al., 1976). The compositional zonation observed in most xenocrystic clinopyroxenes is one of increasing $\mathrm{Al}$ at the expense of NaM2 followed by a later decrease in AlIV (Fig. 4B). The increase in Ti seen in Figure 4B is primarily a function of the decreasing aluminum as opposed to increasing titanium concentration. In the relatively high- 
Table 3. Microprobe analyses of plagioclase, Leg 83.

\begin{tabular}{|c|c|c|c|c|c|c|c|c|c|c|c|c|}
\hline & $\begin{array}{c}(1) \\
83-4^{\mathrm{a}}\end{array}$ & $\begin{array}{c}(2) \\
83-4\end{array}$ & $\begin{array}{c}(3) \\
83-4\end{array}$ & $\begin{array}{c}(4) \\
83-32\end{array}$ & $\begin{array}{c}(5) \\
83-32\end{array}$ & $\begin{array}{c}(6) \\
83-32\end{array}$ & $\begin{array}{c}(7) \\
83-45\end{array}$ & $\begin{array}{c}(8) \\
83-45\end{array}$ & $\begin{array}{c}(9) \\
83-52\end{array}$ & $\begin{array}{c}(10) \\
83-52\end{array}$ & $\begin{array}{c}(11) \\
83-68\end{array}$ & $\begin{array}{c}(12) \\
83-68\end{array}$ \\
\hline $\mathrm{iO}_{2}$ & 47.51 & 48.93 & 46.70 & 48.59 & 47.01 & 46.24 & 46.15 & 46.57 & 45.32 & 46.14 & 47.23 & 47.16 \\
\hline $\mathrm{TiO}_{2}$ & 0.01 & 0.03 & 0.09 & 0.05 & 0.05 & 0.05 & 0.00 & 0.00 & 0.00 & 0.00 & 0.00 & 0.00 \\
\hline $\mathrm{Al}_{2} \overline{\mathrm{O}}_{3}$ & 32.60 & 30.77 & 33.22 & 31.28 & 33.46 & 33.83 & 33.55 & 33.46 & 34.04 & 33.13 & 33.52 & 33.01 \\
\hline $\mathrm{FeO}$ & 0.37 & 0.40 & 0.47 & 0.34 & 0.34 & 0.28 & 0.44 & 0.18 & 0.25 & 0.20 & 0.30 & 0.39 \\
\hline $\mathrm{MgO}$ & 0.23 & 0.31 & 0.22 & 0.34 & 0.28 & 0.27 & 0.23 & 0.25 & 0.22 & 0.22 & 0.26 & 0.26 \\
\hline $\mathrm{CaO}$ & 16.99 & 15.52 & 17.43 & 15.68 & 17.69 & 17.85 & 18.64 & 17.76 & 18.63 & 17.88 & 17.04 & 17.11 \\
\hline $\mathrm{Na}_{2} \mathrm{O}$ & 1.78 & 2.73 & 1.61 & 2.46 & 1.64 & 1.31 & 1.34 & 1.53 & 1.17 & 1.36 & 1.75 & 1.81 \\
\hline $\mathrm{K}_{2} \mathrm{O}$ & 0.01 & 0.00 & 0.00 & 0.03 & 0.03 & 0.04 & 0.00 & 0.00 & 0.01 & 0.01 & 0.00 & 0.01 \\
\hline Total & $99: 50$ & 98.68 & 99.74 & 98.75 & 100.51 & 99.87 & 100.36 & 99.75 & 99.65 & 98.94 & 100.12 & 99.76 \\
\hline \multicolumn{13}{|c|}{ Cations calculated on the basis of 8 oxygens } \\
\hline $\mathrm{Si}$ & 2.193 & 2.270 & 2.156 & 2.2 & 4 & 2.132 & 2.1 & 2.148 & 2.101 & 2.148 & 2.168 & 2.173 \\
\hline $\mathrm{Ti}$ & 0.000 & 0.000 & 0.002 & 0.001 & 0.001 & 0.001 & 0.000 & 0.000 & 0.000 & 0.000 & 0.000 & 0.000 \\
\hline Al & 1.773 & 1.683 & 1.807 & 1.708 & 1.807 & 1.838 & 1.821 & 1.819 & 1.860 & 1.817 & 1.812 & 1.792 \\
\hline $\mathrm{Fe}$ & 0.013 & 0.015 & 0.018 & 0.012 & 0.0 & 0.0 & 0.016 & 0.006 & 0.009 & 0.006 & 0.011 & 0.015 \\
\hline $\mathrm{Mg}$ & 0.015 & 0.020 & 0.015 & 0.023 & 0.019 & 0.018 & 0.015 & 0.016 & 0.015 & 0.015 & 0.017 & 0.018 \\
\hline $\mathrm{Ca}$ & 0.839 & 0.771 & 0.861 & 0.778 & 0.8 & 0.8 & 0.919 & 0.877 & 0.925 & 0.891 & 0.837 & 0.844 \\
\hline $\mathrm{Na}$ & 0.158 & 0.245 & 0.144 & 0.220 & 0.145 & 0.116 & 0.119 & 0.137 & 0.104 & 0.122 & 0.155 & 0.161 \\
\hline K & 0.000 & 0.000 & 0.000 & 0.001 & 0.001 & 0.001 & 0.000 & 0.000 & 0.000 & 0.000 & 0.000 & 0.000 \\
\hline Total & 4.991 & 5.003 & 5.002 & 4.995 & 5.007 & 4.997 & 5.015 & 5.004 & 5.014 & 4.999 & 5.000 & 5.002 \\
\hline $\mathrm{Ab}$ & & & & & & & & & & & 0.156 & 0.160 \\
\hline An & 0.842 & 0.759 & 0.857 & 0.780 & 0.856 & 0.883 & 0.885 & 0.865 & 0.899 & 0.880 & 0.844 & 0.840 \\
\hline Or & 0.000 & 0.000 & 0.000 & 0.000 & 0.001 & 0.001 & 0.000 & 0.000 & 0.000 & 0.000 & 0.000 & 0.000 \\
\hline $\mathrm{Mg} /(\mathrm{Mg}+\mathrm{Fe})$ & 0.536 & 0.571 & 0.454 & 0.657 & 0.613 & 0.621 & 0.484 & 0.727 & 0.625 & 0.714 & 0.607 & 0.545 \\
\hline
\end{tabular}

Note: Numbers that follow are core-section, centimeter ranges of samples from Hole 504B. (1) 73-1, 126-130 cm: subhedral phenocryst core; (2) 73-1, 126-130 cm: core analysis of plagioclase in xenolith composed of plagioclase and clinopyroxene. Plagioclase consists of homogeneous core with porous rim; (3) 73-1, 126-130 cm: boundary between porous rim and homogeneous core; location of maximum An content. Porous rim is normally zoned to $\mathrm{An}_{69}$; (4) 104-2, 24-27 cm: euhedral phenocryst core. Core is unzoned up to thin zone of composition An 86 ; remainder of rim is normally zoned to a composition of $\mathrm{An}_{63}$; (5) Sample 104-2, 24-27 cm: core analysis of xenocryst with numerous small, equant inclusions; (6) 104-2, 24-27 cm: core analysis of xenocryst with abundant large cavities; (7) 123-1, 60-63 cm: core analysis of subhedral xenocryst with numerous cavities located near the center of the crystal; (8) 123-1, 60-63 cm: core analysis of euhedral, lath-shaped phenocryst. Core is unzoned up to a thin zone near the rim having a composition of An 89 ; remainder of rim is normally zoned to $\mathrm{An}_{74}$; (9) 129-1, 76-81 cm: core analysis of subhedral xenocryst/phenocryst with minor development of cavities in the crystal interior. Crystal is oscillatory zoned from An 90 to $\mathrm{An}_{61}$; (10) 129-1, 76-81 cm: core analysis of euhedral phenocryst; (11) 141-1, 16-20 cm: core analysis of euhedral phenocryst. Crystal is oscillatory zoned from An 86 to $\mathrm{An}_{66}$; (12) 141-1, 16-20 cm: core analysis of subhedral phenocryst with large cavities developed throughout the crystal.

a Sample identification number.

Ti basalt, Sample 504B-93-3, 27-32 cm, this is due to the onset of ilmenite crystallization.

Olivine is totally replaced by one or more secondary phyllosilicate minerals, but can be identified by the euhedral morphology of the pseudomorphs. Initially, olivine $0.05-2 \mathrm{~mm}$ in size formed $1-15 \%$ of the rocks.

FeTi-oxides in Hole 504B are predominantly magnetite-ulvospinel $\left(\mathrm{Mt}-\mathrm{Usp}_{\mathrm{ss}}\right)$. Ilmenite co-crystallized with magnetite only in the high Ti, Group $\mathrm{M}^{\prime}$ basalt, Sample 504B-93-3, 27-32 cm. The modal abundance of the oxide phases ranges from 1 to $5 \%$. They occur as euhedral to skeletal crystals confined to the interstitial areas where they formed during the late stages of crystallization (Fig. 5A). In many samples, the oxides are partially or completely altered to sphene (Fig. 5B). Rarely, the Mt$\mathrm{Usp}_{\mathrm{ss}}$ is altered to an intergrowth of ilmenite plus sphene (Fig. 5C). Hole 504B FeTi-oxides are unusual in their high concentration of $\mathrm{MnO}$ - up to 5.19 wt. \% $\mathrm{MnO}$ in primary ilmenite and $4.89 \mathrm{wt} . \%$ in secondary ilmenite intergrown with magnetite (or sphene). Primary magnetite has not been analyzed in an aphyric sample due to its extensive alteration, but magnetite analyzed in phyric basalts is also high in $\mathrm{MnO}$ (Table 2). These Mn concentrations are in the range for acidic intrusive rocks, two to five times higher than anticipated for mid-ocean ridge tholeiites (Haggerty, 1976a). This suggests either that the oxides crystallized late in the crystallization sequence when Mn had been enriched by fractionation or that reequilibration took place over a long period of time to very low temperatures.

\section{Phyric Basalts}

\section{Groundmass}

The phyric basalts from Hole 504B (Leg 83) contain up to $15 \%$ phenocrysts, but most are sparsely to moderately porphyritic (phenocrysts less than $5 \%$ ). Plagioclase is the most abundant phenocryst phase, followed by olivine, followed by clinopyroxene. Groundmass textures range from fine-grained sheaf and plumose quench textures in chilled pillow margins to medium-grained, subophitic/ophitic in dikes and massive units (Figs 6A, 6B). Skeletal to subhedral plagioclase constitutes 30 to $60 \%$ of the groundmass. Clinopyroxenes range from anhedral, equant crystals in intergranular or subophitic relationships with radiate plagioclase clusters to skeletal plumose textures in more rapidly cooled lavas; it forms 30 to $50 \%$ of the groundmass. The groundmass clinopyroxenes are strongly zoned, having up to $22.35 \mathrm{wt} . \%$ $\mathrm{FeO}$ (Fig. 4A) and exhibit more enrichment in titanium 
Table 4. Microprobe analyses of spinels, Leg 83.

\begin{tabular}{|c|c|c|c|c|c|c|c|c|}
\hline & $\stackrel{(1)}{83-32^{\mathrm{a}}}$ & $\begin{array}{c}(2) \\
83-32\end{array}$ & $\begin{array}{c}(3) \\
83-37\end{array}$ & $\begin{array}{c}(4) \\
83-37\end{array}$ & $\begin{array}{c}(5) \\
83-37\end{array}$ & $\begin{array}{c}(6) \\
83-38\end{array}$ & $\begin{array}{c}(7) \\
83-38\end{array}$ & $\begin{array}{c}(8) \\
83-45\end{array}$ \\
\hline $\mathrm{iO}_{2}$ & 0.09 & 0.09 & 0.10 & 0.10 & 0.11 & 0.09 & 0.11 & 0.11 \\
\hline $\mathrm{TiO}_{2}$ & 0.32 & 0.38 & 0.21 & 0.24 & 0.41 & 0.6 & 0.23 & 0.32 \\
\hline $\mathrm{Al}_{2} \bar{O}_{3}$ & 33.72 & 32.03 & 42.29 & 42.75 & 35.28 & 28.25 & 42.15 & 40.18 \\
\hline $\mathrm{Cr}_{2} \mathrm{O}_{3}$ & 31.44 & 33.16 & 24.22 & 22.59 & 29.20 & 38.49 & 22.95 & 24.46 \\
\hline $\mathrm{Fe}_{2} \mathrm{O}_{3}{ }^{*}$ & 5.96 & 7.04 & 5.42 & 5.77 & 6.97 & 4.79 & 5.22 & 6.97 \\
\hline $\mathrm{FeO}$ & 10.43 & 10.14 & 8.89 & 9.53 & 12.18 & 10.34 & 8.17 & 10.16 \\
\hline $\mathrm{MnO}$ & 0.20 & 0.22 & 0.15 & 0.14 & 0.14 & 0.24 & 0.16 & 0.15 \\
\hline $\mathrm{MgO}$ & 17.46 & 17.39 & 19.45 & 18.71 & 16.55 & 16.81 & 19.28 & 18.07 \\
\hline $\mathrm{CaO}$ & 0.05 & 0.06 & 0.05 & 0.09 & 0.07 & 0.04 & 0.07 & 0.13 \\
\hline Total & 99.67 & 100.51 & 100.79 & 99.93 & 100.91 & 99.31 & 98.35 & 100.54 \\
\hline \multicolumn{9}{|c|}{ Cations calculated on the basis of 4 oxygens } \\
\hline $\mathrm{i}$ & 0.001 & 0.001 & 0.002 & 0.002 & 0.002 & 0.001 & 0.002 & 0.002 \\
\hline $\mathrm{Ti}$ & 0.007 & 0.007 & 0.004 & 0.005 & 0.009 & 0.006 & 0.005 & 0.007 \\
\hline $\mathrm{Al}$ & 1.143 & 1.094 & 1.361 & 1.387 & 1.184 & 0.984 & 1.382 & 1.315 \\
\hline $\mathrm{Cr}$ & 0.715 & 0.739 & 0.523 & 0.492 & 0.658 & 0.900 & 0.505 & 0.537 \\
\hline $\mathrm{Fe}^{+3}$ & 0.129 & 0.154 & 0.111 & 0.120 & 0.149 & 0.106 & 0.109 & 0.146 \\
\hline $\mathrm{Fe}^{+2}$ & 0.251 & 0.246 & 0.203 & 0.220 & 0.290 & 0.256 & 0.190 & 0.236 \\
\hline $\mathrm{Mn}$ & 0005 & 0.006 & 0.003 & 0.003 & 0.003 & 0.005 & 0.004 & 0.003 \\
\hline $\mathrm{Mg}$ & 0.749 & 0.752 & 0.792 & 0.768 & 0.703 & 0.741 & 0.800 & 0.749 \\
\hline $\mathrm{Ca}$ & 0.000 & 0.000 & 0.000 & 0.002 & 0.001 & 0.000 & 0.001 & 0.003 \\
\hline Total & 3.000 & 3.000 & 3.000 & 2.999 & 2.999 & 3.000 & 3.000 & 2.999 \\
\hline $\mathrm{Mg} / \mathrm{Mg}+\mathrm{Fe}^{+2}$ & 0.74 & 0.7 & 0.796 & 0.778 & 0.7 & 0.743 & 0.808 & 0.760 \\
\hline $\mathrm{Cr} /$ & 0.385 & 0.403 & 0.278 & 0.262 & 0.357 & 0.478 & 0.268 & 0.290 \\
\hline $\mathrm{Fe}+2 / \mathrm{Fe}+2$ & 0.660 & 0.615 & 0.646 & 0.647 & 0.660 & 0.707 & 0.635 & 0.618 \\
\hline
\end{tabular}

Note: $\mathrm{Fe}_{2} \mathrm{O}_{3} *$ calculated assuming stoichiometry and charge balance. Numbers that follow are core-section, centimeter ranges of samples from Hole 504B. (1) 104-2, 24-27 cm: core composition of small, euhedral crystal enclosed in plagioclase; (2) 104-2, 24-27 cm: rim composition of spinel 1; (3) $111-1,18-24 \mathrm{~cm}$ : core composition of small, euhedral crystal in dike chilled margin; (4) 111-1, 18-24 cm: rim composition of spinel 3; (5) 111-1, 18-24 cm: average composition of large skeletal crystal in dike chilled margin; (6) $111-1,104-106 \mathrm{~cm}$ : core composition of strongly zoned crystal attached to olivine pseudomorph in dike chilled margin; (7) 111-1, 104-106 cm: rim composition of spinel 6; (8) 123-1, 60-63 cm: small euhedral crystal enclosed in plagioclase.

a Sample identification number.

Table 5. Precision of whole-rock basalt chemistry analyses.

\begin{tabular}{|c|c|c|c|c|c|}
\hline & $\mathrm{S}-1^{\mathrm{a}}$ & $S-2^{a}$ & $S-3^{a}$ & & \\
\hline & $\begin{array}{c}77-1, \\
44-53 \mathrm{~cm}\end{array}$ & $\begin{array}{c}97-2, \\
74-89 \mathrm{~cm}\end{array}$ & $\begin{array}{c}130-2, \\
44-58 \mathrm{~cm}\end{array}$ & BCR-1 ${ }^{b}$ & $\begin{array}{l}\text { CRRZ } \\
\text { error }^{\mathrm{c}}\end{array}$ \\
\hline $\mathrm{SiO}_{2}$ & 49.4 & 50.3 & 49.5 & $54.4(0.17)$ & 0.16 \\
\hline $\mathrm{TiO}_{2}$ & 0.95 & 0.97 & 0.78 & $2.25(0.005)$ & 0.005 \\
\hline $\mathrm{Al}_{2} \mathrm{O}_{3}$ & 16.35 & 14.59 & 16.45 & $13.52(0.06)$ & 0.06 \\
\hline $\mathrm{Fe}_{2} \mathrm{O}_{3}{ }^{*}$ & 9.88 & 10.68 & 9.10 & $13.35(0.04)$ & 0.03 \\
\hline $\mathrm{MnO}$ & 0.18 & 0.19 & 0.17 & $0.19(0.01)$ & 0.01 \\
\hline $\mathrm{MgO}$ & 8.33 & 8.43 & 9.13 & $3.34(0.04)$ & 0.04 \\
\hline $\mathrm{CaO}$ & 12.63 & 12.91 & 13.41 & $6.93(0.03)$ & 0.03 \\
\hline $\mathrm{Na}_{2} \mathrm{O}$ & & & & & 0.02 \\
\hline $\mathrm{K}_{2} \mathrm{O}$ & 0.02 & $<0.01$ & 0.01 & $1.69(0.01)$ & 0.01 \\
\hline $\mathrm{P}_{2} \mathrm{O}_{5}$ & 0.08 & 0.08 & 0.08 & $0.38(0.01)$ & 0.01 \\
\hline \multicolumn{6}{|l|}{ Total } \\
\hline $\mathrm{Rb}$ & - & - & - & $46.7(0.6)$ & 0.3 \\
\hline $\mathrm{Sr}$ & 74 & 63 & 63 & 327 (3) & 0.5 \\
\hline Y & - & - & - & $33.8(0.6)$ & 0.2 \\
\hline $\mathrm{Ga}$ & - & - & - & $21.9(0.5)$ & 0.4 \\
\hline $\mathrm{Zr}$ & 50 & 52 & 40 & $193.3(1.4)$ & 0.6 \\
\hline $\mathrm{Nb}$ & 0.6 & 1.1 & 0.7 & $12.4(0.5)$ & 0.4 \\
\hline $\mathrm{Zn}$ & 70 & 71 & 62 & 129 (1) & 1.2 \\
\hline $\mathrm{Ni}$ & 139 & 95 & 162 & $13.8(1.1)$ & 1.1 \\
\hline $\mathrm{Cr}$ & 337 & 277 & 433 & $5.8(1.3)$ & 2.0 \\
\hline $\mathrm{V}$ & 218 & 282 & 217 & 361 (4) & 2.5 \\
\hline
\end{tabular}

a S-1, S-2, and S-3 are whole-rock powders from Leg 83 provided to participants as interlaboratory standards.

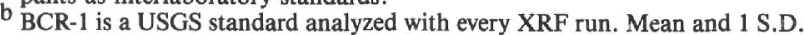
(expressed in wt.\% or ppm; in parentheses) are given.

c Refers to the precision of analysis at the CRRZ concentrations (see text) and is given as 1 S.D. than most aphyric basalt clinopyroxenes. They do not demonstrate the early enrichment in NaM2. Olivine pseudomorphs are present in the groundmass in abundances of 3 to $5 \%$. They are typically less than $0.5 \mathrm{~mm}$ in size and have skeletal to equant, euhedral morphologies. As in the aphyric basalts, the oxides are characterized by an unusually high $\mathrm{MnO}$ content, up to $2.38 \mathrm{wt}$. \% . Primary ilmenite was not conclusively identified in the phyric basalts. However, rare minute crystals of ilmenite (less than $5 \mu \mathrm{m}$ in size), believed to have formed by the oxidation "exsolution" of primary magnetite, were located in altered interstitial areas.

\section{Phenocrysts}

Plagioclase is the most abundant phenocryst phase in phyric basalts from Hole 504B, comprising up to $5 \%$ of the rock (visual estimate). The subhedral to euhedral crystals range from large, equant phenocrysts to lathshaped microphenocrysts. Crystals are up to $3 \mathrm{~mm}$ in size. Glomerocrystic aggregates are extremely common, usually consisting of clumps of 2 to 15 plagioclase crystals in random orientations partially enclosed or enclosing each other. Olivine and/or clinopyroxene occurs with the plagioclase in the glomerocrysts.

Large plagioclase crystals frequently contain cavities or inclusions of devitrified melt. Petrographically, these disequilibrium phenocrysts or xenocrysts are of three types. The first contains abundant, rectangular, devitri- 
Table 6. Chemical composition of basalts from Hole 504B, Leg 83.

\begin{tabular}{|c|c|c|c|c|c|c|c|c|c|c|c|c|c|c|c|c|c|c|c|c|}
\hline & $83-2$ & 83-4 & $83-5$ & $83-6$ & 83-7 & $83-9$ & $83-10$ & $83-11$ & $83-12$ & $83-13$ & $83-14$ & $83-15$ & $83-16$ & $83-17$ & $83-18$ & $83-19$ & $83-20$ & $83-21$ & $83-22$ & $83-23$ \\
\hline $\begin{array}{l}\text { Core-Section } \\
\text { Interval (in cm) }\end{array}$ & $\begin{array}{l}72-1, \\
67-69\end{array}$ & $\begin{array}{c}73-1 \\
126-130\end{array}$ & $\begin{array}{l}76-1 \\
45-47\end{array}$ & $\begin{array}{l}77-3 \\
11-13\end{array}$ & $\begin{array}{l}81-1 \\
64-68\end{array}$ & $\begin{array}{l}82-3 \\
88-92\end{array}$ & $\begin{array}{l}\text { 84-1, } \\
77-79\end{array}$ & $\begin{array}{l}85-2 \\
66-68\end{array}$ & $\begin{array}{l}\text { 87-1, } \\
73-77\end{array}$ & $\begin{array}{l}88-1 \\
109-113\end{array}$ & $\begin{array}{l}89-1 \\
1-4\end{array}$ & $\begin{array}{l}90-1, \\
8-12\end{array}$ & $\begin{array}{l}90-2 \\
23-26\end{array}$ & $\begin{array}{c}90-2 \\
101-104\end{array}$ & $\begin{array}{l}90-4 \\
49-53\end{array}$ & $\begin{array}{c}91-1, \\
127-132\end{array}$ & $\begin{array}{l}91-4, \\
39-43\end{array}$ & $\begin{array}{l}93-1, \\
106-108\end{array}$ & $\begin{array}{l}93-3 \\
27-32\end{array}$ & $\begin{array}{l}94-1, \\
67-72\end{array}$ \\
\hline Depth (m BSF) & 844.18 & 853.78 & 879.96 & 891.62 & 920.16 & 932.40 & 947.28 & 957.67 & 967.75 & 977.61 & 985.54 & 994.60 & 996.24 & 997.03 & 999.51 & 1004.80 & 1008.41 & 1022.57 & 1024.80 & 1031.20 \\
\hline Type $^{\mathrm{a}}$ & $\mathrm{D}^{\prime}$ & $\mathrm{D}^{\prime}$ & $\mathrm{D}^{\prime}$ & $\mathrm{D}^{\prime}$ & $\mathrm{D}^{\prime}$ & $\mathrm{D}^{\prime}$ & $\mathrm{D}^{\prime}$ & $\mathrm{D}^{\prime}$ & $\mathbf{D}^{\prime}$ & $\mathrm{T}$ & $\mathrm{D}^{\prime}$ & $\mathbf{D}^{\prime}$ & $\mathrm{D}^{\prime}$ & $\mathrm{D}^{\prime}$ & $\mathrm{D}^{\prime}$ & $\mathbf{D}^{\prime}$ & $\mathrm{T}$ & $\mathrm{D}^{\prime}$ & $\mathbf{M}^{\prime}$ & $\mathrm{D}^{\prime}$ \\
\hline Group ${ }^{b}$ & OP & OP & OP & A & OPC & OPC & OPC & A & OPC & A & OP & A & $\mathrm{A}$ & A & A & OPC & A & OPC & $\mathrm{A}$ & $\mathrm{OP}$ \\
\hline Alteration ${ }^{\mathrm{c}}$ & 1 & 1 & 1 & $2^{+}$ & 2 & 2 & $2^{+}$ & 2 & 3 & 3 & 3 & 3 & 4 & 2 & 2 & 4 & 3 & 3 & 3 & 1 \\
\hline $\mathrm{SiO}_{2}$ & 49.8 & 49.6 & 49.5 & 50.4 & 49.8 & 49.6 & 49.5 & 50.0 & 50.5 & 49.6 & 49.0 & 49.9 & 49.8 & 50.1 & 49.9 & 49.1 & 51.6 & 50.4 & 48.7 & 49.9 \\
\hline $\mathrm{TiO}_{2}$ & 0.87 & 0.83 & 0.93 & 1.04 & 0.93 & 0.90 & 0.92 & 1.04 & 1.06 & 1.10 & 0.93 & 1.02 & 1.01 & 1.03 & 0.96 & 0.78 & 1.08 & 0.98 & 1.58 & 0.97 \\
\hline $\mathrm{Al}_{2} \mathrm{O}_{3}$ & 16.57 & 15.84 & 16.36 & 14.70 & 15.64 & 15.83 & 15.75 & 15.16 & 14.78 & 15.45 & 16.18 & 14.90 & 14.77 & 14.45 & 16.08 & 16.00 & 15.10 & 15.46 & 16.00 & 15.43 \\
\hline $\mathrm{Fe} 2 \mathrm{O}_{3}{ }^{* \mathrm{~d}}$ & 9.13 & 9.46 & 9.83 & 10.68 & 10.05 & 9.69 & 9.73 & 10.46 & 10.34 & 9.96 & 9.41 & 10.18 & 10.55 & 11.47 & 9.67 & 9.82 & 9.75 & 9.93 & 10.71 & 10.31 \\
\hline $\mathrm{MnO}$ & 0.15 & $\begin{array}{l}9.40 \\
0.17\end{array}$ & $\begin{array}{l}9.19 \\
0.19\end{array}$ & $\begin{array}{r}10.00 \\
0.18\end{array}$ & 0.27 & $\begin{array}{l}9.09 \\
0.26\end{array}$ & 0.25 & $\begin{array}{r}10.40 \\
0.22\end{array}$ & $\begin{array}{r}10.34 \\
0.27\end{array}$ & $\begin{array}{l}9.24 \\
0.24\end{array}$ & $\begin{array}{l}9.41 \\
0.24\end{array}$ & $\begin{array}{r}10.10 \\
0.25\end{array}$ & 0.26 & $\begin{array}{r}1.47 \\
0.22\end{array}$ & $\begin{array}{l}9.01 \\
0.21\end{array}$ & $\begin{array}{l}9.82 \\
0.24\end{array}$ & 0.25 & 0.23 & 0.21 & $\begin{array}{l}0.17 \\
0.17\end{array}$ \\
\hline $\mathrm{MgO}$ & 8.55 & 8.99 & 8.88 & 8.29 & 8. & 9.0 & 8.71 & 8.53 & 8.08 & 8. & 8.96 & 8.96 & 9.0 & 8. & 8.6 & 8 & 8. & 8. & 8. & 8.42 \\
\hline $\mathrm{CaO}$ & 13.12 & 12.93 & $\begin{array}{l}0.00 \\
12.72\end{array}$ & 12.25 & 13.21 & 13.12 & $\begin{array}{l}13.18 \\
\end{array}$ & 13.18 & 12.98 & $\begin{array}{l}0.00 \\
12.74\end{array}$ & 13.16 & $\begin{array}{l}13.06 \\
13.06\end{array}$ & 12.58 & 13.00 & $\begin{array}{l}0.03 \\
12.67\end{array}$ & $\begin{array}{l}13.61 \\
13.61\end{array}$ & 11.74 & 12.87 & 11.88 & 12.70 \\
\hline $\mathrm{Na}_{2} \mathrm{O}$ & 2.22 & $1.93^{\mathrm{e}}$ & 2.16 & $2.44^{\mathrm{e}}$ & 1.96 & 1.83 & $1.87^{\mathrm{e}}$ & 1.95 & 2.19 & $2.13^{\mathrm{e}}$ & 2.19 & 1.99 & 1.90 & 1.82 & $1.74^{\mathrm{e}}$ & $1.65^{\mathrm{e}}$ & 2.17 & 2.08 & $2.34^{\mathrm{e}}$ & 1.92 \\
\hline $\mathrm{K}_{2} \mathrm{O}$ & 0.03 & 0.04 & 0.02 & 0.02 & 0.01 & 0.02 & 0.01 & 0.03 & 0.03 & 0.05 & 0.02 & 0.01 & 0.01 & 0.01 & 0.02 & 0.01 & 0.01 & 0.02 & 0.01 & 0.01 \\
\hline $\mathrm{P}_{2} \mathrm{O}_{5}$ & 0.09 & 0.07 & 0.09 & 0.08 & 0.08 & 0.09 & 0.09 & 0.10 & 0.10 & 0.13 & 0.08 & 0.10 & 0.09 & 0.09 & 0.09 & 0.08 & 0.09 & 0.09 & 0.19 & 0.07 \\
\hline Total & 100.53 & 99.86 & 100.68 & 100.08 & 100.36 & 100.34 & 100.01 & 100.67 & 100.33 & 100.23 & 100.17 & 100.37 & 100.06 & 100.42 & 100.03 & 100.20 & 100.38 & 100.34 & 100.28 & 99.90 \\
\hline $\mathrm{Rb}$ & 0.6 & $<0.5$ & 0.5 & 0.6 & $<0.5$ & $<0.5$ & $<0.5$ & $<0.5$ & $<0.5$ & $<0.5$ & 0.5 & $<0.5$ & $<0.5$ & $<0.5$ & 0.6 & 0.5 & $<0.5$ & $<0.5$ & $<0.5$ & $<0.5$ \\
\hline Sr & 65 & 61 & 72 & 56 & 54 & 49 & 51 & 62 & 59 & 75 & 69 & 54 & 55 & 47 & 66 & 39 & 72 & 60 & 76 & 57 \\
\hline Y & 21.1 & 20.3 & 22.2 & 26.4 & 23.1 & 22.3 & 22.7 & 25.5 & 26.7 & 24.2 & 22.4 & 25.5 & 25.1 & 26.8 & 22.6 & 20.4 & 22.8 & 23.2 & 33.0 & 23.9 \\
\hline $\mathrm{Ga}$ & 13.8 & 15.0 & 14.8 & 15.5 & 15.2 & 15.4 . & 14.8 & 15.6 & 14.1 & 15.0 & 14.1 & 14.5 & 15.2 & 16.0 & 14.6 & 14.8 & 14.5 & 15.0 & 16.4 & 15.9 \\
\hline $\mathrm{Zr}$ & 44 & 42 & 45 & 54 & 48 & 46 & 46 & 56 & 54 & 68 & 55 & 52 & 54 & 52 & 54 & 36 & 67 & 53 & 132 & 50 \\
\hline $\mathrm{Nb}$ & 0.8 & 0.6 & $<0.5$ & 0.7 & $\begin{array}{c}40 \\
0.6\end{array}$ & $\begin{array}{c}40 \\
0.7\end{array}$ & $<0.5$ & 0.7 & 0.8 & $\begin{array}{c}00 \\
3.8\end{array}$ & 0.5 & $<0.5$ & 0.5 & 0.7 & 0.6 & 0.6 & 4.2 & 1.1 & 1.4 & 0.9 \\
\hline $\mathrm{Zn}$ & 76 & 68 & 67 & 85 & 102 & 97 & 99 & 92 & 108 & 104 & 97 & 98 & 98 & 87 & 88 & 102 & 109 & 89 & 89 & 84 \\
\hline $\mathrm{Ni}$ & 150 & $\begin{array}{r}150 \\
\text { - }\end{array}$ & 166 & 92 & 116 & 138 & 121 & 101 & 82 & 105 & 13 & 95 & 99 & 87 & 102 & 132 & 108 & 97 & 134 & 117 \\
\hline $\mathrm{Cr}$ & $\begin{array}{l}398 \\
398\end{array}$ & 437 & 381 & 242 & 377 & 393 & 373 & 351 & 205 & 330 & 427 & 308 & 352 & 204 & $\begin{array}{l}102 \\
355\end{array}$ & $\begin{array}{l}132 \\
424\end{array}$ & 353 & 338 & 287 & 312 \\
\hline $\mathrm{v}$ & 242 & 230 & 227 & 311 & 283 & 264 & 275 & 281 & 278 & 256 & 251 & 305 & 294 & 308 & 250 & 262 & 257 & 270 & 236 & 269 \\
\hline mg value $\mathrm{f}^{\mathrm{f}}$ & 0.673 & 0.677 & 0.665 & 0.631 & 0.648 & 0.672 & 0.663 & 0.642 & 0.632 & 0.661 & 0.677 & 0.660 & 0.655 & 0.612 & 0.664 & 0.666 & 0.660 & 0.648 & 0.640 & 0.643 \\
\hline $\mathrm{FeO}^{*}$ & 8.22 & 8.5 & 8.8 & 9.6 & 9.0 & 8.7 & 8.7 & 9.4 & 9.3 & 8.9 & 8.47 & 9.16 & 9.4 & 10.3 & 8.70 & 8.8 & 8.77 & 8.94 & 9.64 & 9.28 \\
\hline $\mathrm{FeO}^{*} / \mathrm{Mg}_{8}$ & $\begin{array}{l}0.22 \\
0.96\end{array}$ & $\begin{array}{l}8.95 \\
0.95\end{array}$ & $\begin{array}{l}8.03 \\
1.00\end{array}$ & $\begin{array}{l}.01 \\
1.16\end{array}$ & $\begin{array}{l}1.04 \\
1.07\end{array}$ & 0.97 & $\begin{array}{l}8.00 \\
1.00\end{array}$ & $\begin{array}{l}.4 .41 \\
1.10\end{array}$ & $\begin{array}{l}9.30 \\
1.15\end{array}$ & $\begin{array}{l}8.90 \\
1.01\end{array}$ & $\begin{array}{l}8.41 \\
0.95\end{array}$ & $\begin{array}{l}\text {.1. } \\
1.02\end{array}$ & $\begin{array}{l}9.49 \\
1.04\end{array}$ & $\begin{array}{l}0.22 \\
1.25\end{array}$ & 1.00 & $\begin{array}{l}0.84 \\
0.99\end{array}$ & $\begin{array}{l}8.02 \\
1.11\end{array}$ & $\begin{array}{l}1.94 \\
1.08\end{array}$ & $\begin{array}{l}9.04 \\
1.11\end{array}$ & $\begin{array}{l}9.28 \\
1.10\end{array}$ \\
\hline $\mathrm{CaO}$ & 5.9 & 6.7 & 6.9 & 5.0 & 6.7 & 7.2 & 7.0 & 6. & 5. & 6. & 6. & & 6. & 7. & 7. & 8. & 5. & 6.2 & 5.1 & 6.6 \\
\hline $\mathrm{Zr} / \mathrm{Y}$ & 2.06 & 2.08 & 2.21 & 2.03 & 2.06 & 2.04 & 2.01 & 2.20 & 2.02 & 2.82 & 2.44 & 2.02 & 2.16 & 1.93 & 2.37 & $\begin{array}{l}0.2 \\
1.76\end{array}$ & 2.92 & 2.28 & 4.00 & $\begin{array}{l}0.08 \\
2.08\end{array}$ \\
\hline
\end{tabular}

Note: The first number in each column head represents the laboratory sample identification number.

a Type refers to chemical units discussed in text.

$\mathrm{b}$ Group refers to petrographic groups defined in text.

c Alteration refers to the alteration index defined in text. $\mathrm{Fe}^{2+}$

${ }^{\mathrm{d}} \mathrm{Fe}_{2} \mathrm{O}_{3}{ }^{*}$ and $\mathrm{FeO}^{*}$ refer to total $\mathrm{Fe}$ expressed as $\mathrm{Fe}^{3+}$ or $\mathrm{Fe}^{2+}$

f $\mathrm{Calculation}$ of $\mathrm{mg}$-value is discussed in the text. 
Table 6. (Continued).

\begin{tabular}{|c|c|c|c|c|c|c|c|c|c|c|c|c|c|c|c|c|c|c|c|c|}
\hline & $83-24$ & $83-25$ & $83-26$ & $83-27$ & $83-28$ & $83-30$ & $83-31$ & $83-32$ & $83-33$ & $83-34$ & $83-35$ & $83-36$ & $83-39$ & $83-40$ & $83-41$ & $83-42$ & $83-42$ & $83-44$ & $83-45$ & $83-47$ \\
\hline $\begin{array}{l}\text { Core-Section } \\
\text { Interval (in cm) }\end{array}$ & $\begin{array}{c}\text { 94-3, } \\
143-147\end{array}$ & $\begin{array}{c}95-1 \\
137-140\end{array}$ & $\begin{array}{c}99-1, \\
112-116\end{array}$ & $\begin{array}{l}100-2, \\
26-31\end{array}$ & $\begin{array}{c}101-1 \\
2-5\end{array}$ & $\begin{array}{l}101-2 \\
16-20\end{array}$ & $\begin{array}{l}104-1 \\
15-19\end{array}$ & $\begin{array}{l}104-2 \\
24-27\end{array}$ & $\begin{array}{l}\text { 107-2, } \\
71-75\end{array}$ & $\begin{array}{l}\text { 108-1, } \\
95-97\end{array}$ & $\begin{array}{l}109-1 \\
49-52\end{array}$ & $\begin{array}{l}110-1 \\
17-19\end{array}$ & $\begin{array}{l}112-1 \\
34-36\end{array}$ & $\begin{array}{c}113-1 \\
129-133\end{array}$ & $\begin{array}{l}116-1 \\
43-46\end{array}$ & $\begin{array}{l}\text { 117-1, } \\
9-13\end{array}$ & $\begin{array}{l}118-1 \\
48-53\end{array}$ & $\begin{array}{l}121-1 \\
15-19\end{array}$ & $\begin{array}{l}123-1 \\
60-63\end{array}$ & $\begin{array}{l}124-1 \\
98-100\end{array}$ \\
\hline Depth (m BSF) & 1034.95 & 1040.89 & $\begin{array}{l}1072.64 \\
\text { lat }\end{array}$ & 1082.28 & 1089.53 & 1091.18 & 1116.67 & 1118.26 & 1145.73 & $\begin{array}{l}93.91 \\
1153.46\end{array}$ & $\begin{array}{c}49-32 \\
1154.01\end{array}$ & $\begin{array}{c}1157.18 \\
1157.18\end{array}$ & $\begin{array}{c}34-36 \\
1166.35\end{array}$ & $\begin{array}{l}129-133 \\
1172.31\end{array}$ & $\begin{array}{c}43-46 \\
1185.45\end{array}$ & $\begin{array}{c}9-13 \\
1189.61\end{array}$ & $\begin{array}{c}48-33 \\
1194.51\end{array}$ & $\begin{array}{l}1207.67 \\
1207.67\end{array}$ & $\begin{array}{c}\text { ov-63 } \\
1223.12\end{array}$ & $\begin{array}{l}98-100 \\
1232.49\end{array}$ \\
\hline Type $^{\mathrm{a}}$ & $\mathrm{D}^{\prime}$ & $\mathrm{D}^{\prime}$ & $\mathrm{D}^{\prime}$ & $\mathrm{D}^{\prime}$ & $\mathrm{D}^{\prime}$ & $\mathrm{D}^{\prime}$ & $\mathrm{D}^{\prime}$ & $\mathrm{D}^{\prime}$ & $\mathrm{D}^{\prime}$ & $\mathrm{D}^{\prime}$ & $\mathrm{D}^{\prime}$ & $\mathrm{D}^{\prime}$ & $\mathrm{D}^{\prime}$ & $\mathrm{D}^{\prime}$ & $\mathrm{D}^{\prime}$ & $\mathrm{D}^{\prime}$ & $\mathrm{D}^{\prime}$ & $D^{\prime}$ & $\mathrm{D}^{\prime}$ & $\mathrm{D}^{\prime}$ \\
\hline Group $b$ & A & OPC & A & OPC & A & A & OPC & OPC & A & A & OPC & A & OP & OP & OPC & OPC & OPC & A & OP & OPC \\
\hline Alteration ${ }^{c}$ & 3 & 4 & $2^{+}$ & $2^{+}$ & $2^{+}$ & 2 & 2 & 3 & 1 & 1 & 3 & 2 & 1 & 3 & 4 & 2 & 2 & 3 & 1 & 3 \\
\hline $\mathrm{SiO}_{2}$ & 50.2 & 51.7 & 50.1 & 50.1 & 49.9 & 50.6 & 50.1 & 50.0 & 50.6 & 50.8 & 50.3 & 50.4 & 50.0 & 50.3 & 49.0 & 50.7 & 50.6 & 50.7 & 48.8 & 50.4 \\
\hline $\mathrm{TiO}_{2}$ & 1.06 & 1.01 & 0.94 & 0.90 & 0.90 & 1.12 & 1.03 & 0.95 & 0.84 & 0.89 & 0.93 & 0.90 & 0.81 & 0.82 & 0.98 & 0.97 & 0.97 & 1.05 & 0.85 & 0.96 \\
\hline $\mathrm{Al}_{2} \mathrm{O}_{3}$ & 15.04 & 14.51 & 14.65 & 14.98 & 14.77 & 14.31 & 14.85 & 16.01 & 15.11 & 14.76 & 15.54 & 14.91 & 15.30 & 15.38 & 15.01 & 15.29 & 15.11 & 14.45 & 16.50 & 14.86 \\
\hline $\mathrm{Fe}_{2} \mathrm{O}_{3}$ *d & 11.01 & 10.62 & 10.79 & 10.24 & 10.40 & 11.62 & 11.01 & 9.84 & $\begin{array}{l}9.78 \\
\end{array}$ & 10.11 & 10.22 & 9.97 & 9.88 & 9.87 & 10.79 & 10.40 & 10.49 & 11.27 & 9.90 & 10.60 \\
\hline $\mathrm{MnO}^{2}$ & 0.22 & 0.20 & 0.21 & 0.19 & 0.20 & 0.20 & 0.19 & 0.17 & 0.17 & 0.17 & 0.18 & 0.19 & 0.17 & 0.17 & 0.18 & 0.17 & 0.17 & 0.19 & 0.17 & 0.20 \\
\hline $\mathrm{MgO}$ & 7.87 & 8.11 & 8.79 & 8.65 & 8.60 & 7.72 & 8.05 & 7.97 & 8.86 & 8.79 & 8.01 & 8.63 & 9.05 & 8.75 & 8.81 & 8.09 & 8.38 & 7.99 & 8.67 & 8.42 \\
\hline $\mathrm{CaO}$ & 12.78 & 12.29 & 12.94 & 13.37 & 13.01 & 12.61 & 12.69 & 13.05 & 13.06 & 12.86 & 13.03 & 13.11 & 13.34 & 13.32 & 12.67 & 13.10 & 12.95 & 12.51 & 13.20 & 13.14 \\
\hline $\mathrm{Na}_{2} \mathrm{O}$ & 1.86 & 1.95 & 2.10 & 1.85 & 2.33 & 1.98 & 1.76 & $1.84^{\mathrm{e}}$ & 2.07 & 1.96 & $1.86^{\mathrm{e}}$ & 2.14 & 1.75 & $1.64^{\mathrm{e}}$ & $2.52^{\mathrm{e}}$ & 1.81 & 1.90 & 1.85 & 1.88 & 1.87 \\
\hline $\mathrm{K}_{2} \mathrm{O}$ & 0.02 & 0.03 & 0.01 & 0.01 & 0.02 & 0.01 & 0.01 & 0.01 & 0.01 & 0.03 & 0.01 & 0.01 & 0.17 & 0.01 & 0.02 & 0.01 & 0.01 & 0.01 & 0.01 & 0.04 \\
\hline $\mathrm{P}_{2} \mathrm{O}_{5}$ & 0.10 & 0.09 & 0.09 & 0.08 & 0.08 & 0.10 & 0.10 & 0.09 & 0.08 & 0.08 & 0.10 & 0.09 & 0.07 & 0.07 & 0.09 & 0.09 & 0.09 & 0.10 & 0.08 & 0.12 \\
\hline Total & 100.16 & 100.51 & 100.62 & 100.37 & 100.21 & 100.27 & 99.79 & 99.93 & 100.58 & 100.45 & 100.18 & 100.35 & 100.54 & 100.33 & 100.07 & 100.63 & 100.67 & 100.12 & 100.06 & 100.61 \\
\hline $\mathrm{Rb}$ & 0.5 & $<0.5$ & 0.5 & $<0.5$ & $<0.5$ & $<0.5$ & 0.7 & 0.5 & $<0.5$ & 0.9 & $<0.5$ & $<0.5$ & $<0.5$ & $<0.5$ & $<0.5$ & $<0.5$ & $<0.5$ & $<0.5$ & $<0.5$ & $<0.5$ \\
\hline Sr & 47 & 51 & 49 & 48 & 46 & 52 & 54 & 61 & 58 & 59 & 50 & 57 & 51 & 43 & 49 & 49 & 51 & 43 & 60 & 45 \\
\hline Y & 26.7 & 25.2 & 24.1 & 23.6 & 23.7 & 28.0 & 25.8 & 22.9 & 20.5 & 22.4 & 23.3 & 22.5 & 20.3 & 20.1 & 24.8 & 24.7 & 24.5 & 26.9 & 23.0 & 24.5 \\
\hline $\mathrm{Ga}$ & 15.4 & 15.0 & 14.9 & 16.0 & 15.0 & 16.1 & 16.8 & 16.4 & 15.7 & 15.3 & 15.9 & 15.2 & 15.4 & 15.0 & $\begin{array}{l}2.6 \\
15.9\end{array}$ & 15.7 & 15.5 & 16.0 & 15.7 & 16.1 \\
\hline $\mathrm{Zr}$ & $\begin{array}{l}1.4 \\
54\end{array}$ & $\begin{array}{l}51.0 \\
\text { s. }\end{array}$ & $\begin{array}{l}11.9 \\
45\end{array}$ & 43 & 38 & $\begin{array}{l}10.1 \\
56\end{array}$ & $\begin{array}{l}10.0 \\
51\end{array}$ & $\begin{array}{l}10.4 \\
49\end{array}$ & 43 & 46 & $\begin{array}{l}13.9 \\
46\end{array}$ & $\begin{array}{l}15.2 \\
45\end{array}$ & 38 & 38 & 44 & 47 & 47 & $\begin{array}{l}10.0 \\
49\end{array}$ & 43 & $\begin{array}{l}10.1 \\
43\end{array}$ \\
\hline $\mathrm{Nb}$ & 0.5 & 0.5 & 0.8 & 0.8 & 1.1 & $<0.5$ & 0.6 & 0.9 & 0.5 & 0.5 & $<0.5$ & 0.9 & 0.7 & 0.7 & 0.5 & 0.7 & 1.1 & 0.5 & 1.0 & 0.7 \\
\hline $\mathrm{Zn}$ & 104 & 79 & 80 & 77 & 81 & 84 & 79 & 70 & 67 & 75 & 72 & 71 & 84 & 79 & 50 & 79 & 73 & 94 & 75 & 66 \\
\hline $\mathrm{Ni}$ & 91 & 89 & 92 & 10 & 87 & 76 & 90 & 10 & 10 & 99 & 90 & 99 & 124 & 116 & 99 & 98 & 103 & 90 & 137 & 92 \\
\hline $\mathrm{Cr}$ & 256 & 209 & 228 & 4 & 210 & 168 & 228 & 363 & 333 & 314 & 267 & 315 & 427 & 384 & 342 & 292 & 297 & 218 & 178 & 339 \\
\hline $\mathrm{v}$ & 303 & 280 & 292 & 283 & 302 & 319 & 282 & 246 & 245 & 262 & 272 & 256 & 249 & 254 & 286 & 294 & 286 & 320 & 240 & 281 \\
\hline mg value $\mathrm{f}^{\mathrm{f}}$ & 0.611 & 0.627 & 0.642 & 0.650 & 0.645 & 0.594 & 0.617 & 0.641 & 0.666 & 0.657 & 0.633 & 0.656 & 0.668 & 0.661 & 0.642 & 0.631 & 0.637 & 0.609 & 0.658 & 0.636 \\
\hline $\mathrm{FeO}^{*}$ & 9. & 9 & 9. & 9. & 9 & 10. & 9 & 8. & 8. & 9. & 9.2 & 8. & 8. & 8. & 9. & 9. & 9. & 10.1 & 8.91 & 9.54 \\
\hline $\mathrm{FeO} * / \mathrm{MgO}$ & 1.26 & 1.18 & 1.11 & 1.07 & 1.08 & 1.36 & 1.23 & 1.11 & 0.99 & 1.03 & 1.1 & 1.03 & 0.9 & $\begin{array}{l}0.00 \\
1.01\end{array}$ & 1.1 & 1.15 & 1.1 & 1.26 & 1.02 & 1.14 \\
\hline $\mathrm{CaO} / \mathrm{Na}$ O & $\begin{array}{l}6.20 \\
6.9\end{array}$ & 6.3 & 6.2 & 7.2 & $\begin{array}{l}5.60 \\
5.6\end{array}$ & 6.4. & 7.2 & 7.1 & $\begin{array}{l}6.99 \\
6.3\end{array}$ & 6. & 7. & 6. & 7 & $\begin{array}{l}8.0 \\
8 .\end{array}$ & $\begin{array}{r}1.1 \\
5\end{array}$ & 7.2 & $\begin{array}{l}1.12 \\
6.8\end{array}$ & $\begin{array}{r}1.20 \\
6.8\end{array}$ & 7.0 & $\begin{array}{r}1.14 \\
7.0\end{array}$ \\
\hline $\mathrm{Zr} / \mathrm{Y}$ & 2.03 & 2.02 & 1.85 & 1.81 & 1.61 & 2.00 & 1.98 & 2.14 & 2.08 & 2.04 & 1.97 & 2.00 & 1.88 & 1.91 & 1.77 & 1.89 & 1.92 & 1.81 & 1.88 & 1.77 \\
\hline
\end{tabular}


Table 6. (Continued).

\begin{tabular}{|c|c|c|c|c|c|c|c|c|c|c|c|c|c|c|c|c|c|c|c|}
\hline & $83-48$ & $83-49$ & $83-50$ & $83-51$ & $83-52$ & $83-53$ & $83-55$ & $83-56$ & $83-57$ & 83-58 & $83-59$ & 83.60 & $83-61$ & $83-62$ & $83-63$ & $83-64$ & $83-65$ & $83-67$ & $83-68$ \\
\hline $\begin{array}{l}\text { Core-Section } \\
\text { Interval (in } \mathrm{cm} \text { ) }\end{array}$ & $\begin{array}{l}125-1 \\
32-36\end{array}$ & $\begin{array}{l}126-1 \\
38-41\end{array}$ & $\begin{array}{l}127-1 \\
38-42\end{array}$ & $\begin{array}{l}128-1 \\
115-118\end{array}$ & $\begin{array}{l}129-1 \\
76-81\end{array}$ & $\begin{array}{c}129-2 \\
130-132\end{array}$ & $\begin{array}{l}130-1 \\
15-20\end{array}$ & $\begin{array}{l}\text { 130-3 } \\
94-99\end{array}$ & $\begin{array}{c}131-1 \\
3-7\end{array}$ & $\begin{array}{c}132-1 \\
141-144\end{array}$ & $\begin{array}{l}133-1 \\
10-15\end{array}$ & $\begin{array}{c}\text { 133-1, } \\
101-106\end{array}$ & $\begin{array}{l}\text { 134-1, } \\
72-74\end{array}$ & $\begin{array}{c}136-1 \\
4-7\end{array}$ & $\begin{array}{l}137-1 \\
11-16\end{array}$ & $\begin{array}{l}\text { 137-1, } \\
93-97\end{array}$ & $\begin{array}{l}138-1 \\
62-67\end{array}$ & $\begin{array}{l}140-1 \\
40-42\end{array}$ & $\begin{array}{l}141-1 \\
16-20\end{array}$ \\
\hline Depth (m BSF) & 1240.84 & 1249.90 & 1253.90 & 1262.17 & 1270.78 & 1272.81 & 1279.17 & 1282.97 & 1287.55 & 1296.42 & 1304.13 & 1305.03 & 1313.73 & 1322.06 & 1327.14 & $\begin{array}{l}1327.95 \\
\end{array}$ & 1332.65 & 1341.41 & 1345,68 \\
\hline Type $^{a}$ & $\mathrm{D}^{\prime}$ & $\mathrm{D}^{\prime}$ & $\mathrm{D}^{\prime}$ & $\mathrm{D}^{\prime}$ & $\mathrm{D}^{\prime}$ & $\mathrm{D}^{\prime}$ & $\mathrm{D}^{\prime}$ & $\mathrm{D}^{\prime}$ & $\mathrm{D}^{\prime}$ & $\mathrm{D}^{\prime}$ & $\mathrm{D}^{\prime}$ & $\mathrm{D}^{\prime}$ & $\mathrm{D}^{\prime}$ & $\mathrm{D}^{\prime}$ & $\mathrm{D}^{\prime}$ & $\mathrm{D}^{\prime}$ & $\mathrm{D}^{\prime}$ & $\mathrm{D}^{\prime}$ & $\mathrm{D}^{\prime}$ \\
\hline Group ${ }^{b}$ & A & OPC & OPC & OPC & OPC & A & OPC & OPC & $\mathrm{OPC}$ & OPC & OP & OPC & OPC & A & OPC & OPC & OPC & A & OPC \\
\hline Alteration ${ }^{\mathrm{c}}$ & - & 2 & 2 & 2 & 3 & 3 & 2 & 2 & 4 & 3 & $2^{+}$ & $2^{+}$ & $2^{+}$ & - & 2 & 3 & 2 & 3 & 3 \\
\hline $\mathrm{SiO}_{2}$ & 49.8 & 49.3 & 49.8 & 48.9 & 49.4 & 50.1 & 49.5 & 48.9 & 49.4 & 49.3 & 49.5 & 50.0 & 49.7 & 50.0 & 49.5 & 49.0 & 49.7 & 48.8 & 49.3 \\
\hline $\mathrm{TiO}_{2}$ & 1.01 & 0.76 & 0.77 & 0.74 & 0.71 & 1.05 & 0.75 & 0.73 & 0.74 & 0.86 & 0.86 & 0.96 & 0.98 & 1.09 & 0.79 & 0.78 & 0.82 & 1.14 & 0.84 \\
\hline $\mathrm{Al}_{2} \mathrm{O}_{3}$ & 14.72 & 16.45 & 16.32 & 16.36 & 15.80 & 14.66 & 16.46 & 16.15 & 16.57 & 15.89 & 16.29 & 14.65 & 15.48 & 14.44 & 16.23 & 17.19 & 16.21 & 14.70 & 16.35 \\
\hline $\mathrm{Fe}_{2} \mathrm{O}_{3}{ }^{* \mathrm{~d}}$ & 10.96 & 8.95 & 9.19 & 8.93 & 9.14 & 11.00 & 9.06 & 9.19 & 8.83 & 9.91 & 9.87 & 10.64 & 10.42 & 11.28 & 9.30 & 8.89 & 9.27 & 11.59 & 9.37 \\
\hline MnO & 0.23 & 0. & 0 & 0.14 & 0.15 & 0.21 & 0.16 & 0.15 & 0.16 & 0.18 & 0.17 & 0.19 & 0.17 & 0.19 & 0.18 & 0.16 & 0.16 & 0.22 & 0.16 \\
\hline $\mathrm{MgO}$ & 8.51 & 8.89 & 9.51 & 9.34 & 10.65 & 8.31 & 9.23 & 10.43 & 9.51 & 8.57 & 8.36 & 8.34 & 8.18 & 8.08 & 9.19 & 8.52 & 8.65 & 8.44 & 8.40 \\
\hline $\mathrm{CaO}$ & 13.16 & 13.28 & 13.29 & 13.39 & 12.93 & 12.93 & 13.37 & 12.81 & 13.41 & 13.39 & 13.43 & 13.02 & 12.98 & 12.48 & 13.35 & 13.69 & 13.36 & 12.99 & 13.26 \\
\hline $\mathrm{Na}_{2} \mathrm{O}$ & 2.07 & 1.75 & 1.71 & 1.63 & $1.48^{\mathrm{e}}$ & 2.15 & 1.76 & 1.61 & 1.65 & 1.83 & 1.85 & $1.89^{\mathrm{e}}$ & 1.95 & 2.00 & 1.81 & 1.82 & 1.85 & $1.68^{\mathrm{e}}$ & $1.96^{\mathrm{e}}$ \\
\hline $\mathrm{K}, \mathrm{O}$ & 0.02 & 0.01 & 0.01 & 0.01 & $<0.01$ & 0.01 & 0.01 & $<0.01$ & 0.01 & 0.01 & 0.01 & 0.02 & 0.01 & 0.02 & 0.01 & 0.01 & 0.02 & 0.03 & 0.03 \\
\hline $\mathrm{P}_{2} \mathrm{O}_{5}$ & 0.09 & 0.08 & 0.08 & 0.08 & 0.07 & 0.09 & 0.07 & 0.08 & 0.06 & 0.07 & 0.08 & 0.08 & 0.08 & 0.10 & 0.07 & 0.07 & 0.07 & 0.11 & 0.07 \\
\hline Total & 100.57 & 99.62 & 100.83 & 99.52 & 100.33 & 100.51 & 100.37 & 100.06 & 100.34 & 100.01 & 100.42 & 99.79 & 99.95 & 99.68 & 100.43 & 100.13 & 100.11 & 99.70 & 99.74 \\
\hline $\mathrm{Rb}$ & $<0.5$ & $<0.5$ & $<0.5$ & $<0.5$ & $<0.5$ & $<0.5$ & $<0.5$ & $<0.5$ & $<0.5$ & $<0.5$ & $<0.5$ & $<0.5$ & $<0.5$ & 0.6 & $<0.5$ & $<0.5$ & $<0.5$ & $<0.5$ & $<0.5$ \\
\hline $\mathrm{Sr}$ & 48 & 64 & 61 & 60 & 54 & 58 & 62 & 55 & 58 & 57 & 59 & 45 & 58 & 58 & 61 & 61 & 59 & 51 & 60 \\
\hline Y & 25.9 & 18. & 18.8 & 18.1 & 17.2 & 25.7 & 18.2 & 18.2 & 18.1 & 22.5 & 22.3 & 24. & 23.9 & 26.6 & 19.3 & 19.4 & 20. & 20.8 & 20.6 \\
\hline $\mathrm{Ga}$ & 15.2 & 14.7 & 15.0 & 14.8 & 13.1 & 16.4 & 15.1 & 14.4 & 13.9 & 14.8 & 15.4 & 15.6 & 15.9 & 15.6 & 14.3 & 14.7 & 14.5 & 15.4 & 15.8 \\
\hline $\mathrm{Zr}$ & 48 & 40 & 40 & 37 & 36 & 56 & 39 & 37 & 38 & 44 & 44 & 45 & 49 & 58 & 42 & 40 & 41 & 59 & 42 \\
\hline $\mathrm{Nb}$ & 0.7 & 0.8 & 0.7 & 0.8 & 0.5 & 0.6 & 0.5 & 0.6 & 0.9 & 0.8 & 0.8 & 0.7 & 0.9 & 1.0 & 0.9 & 0.7 & 0.6 & 1.0 & 0.6 \\
\hline $\mathrm{Zn}$ & 90 & 77 & 66 & 66 & 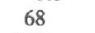 & 73 & 6 & 65 & 76 & 0.0 & 74 & 0. & 0. & 101 & 73 & 0.1 & 0.0 & 71 & 66 \\
\hline $\mathrm{Ni}$ & 92 & 15 & 16 & 16 & 24 & 8 & 160 & 21 & 15 & 12 & 122 & 1 & 18 & 84 & 154 & 14 & 1 & 91 & 120 \\
\hline $\mathrm{Cr}$ & 323 & & 4 & 4 & 5 & 2 & & 3 & 451 & 3 & 382 & 317 & 2 & 177 & 424 & 457 & 417 & 267 & 384 \\
\hline v & 296 & 220 & 219 & 217 & 204 & 298 & 218 & 196 & 167 & 261 & 256 & 287 & 269 & 307 & 225 & 222 & 233 & 326 & 239 \\
\hline$m g$ value $\mathrm{f}^{\mathrm{f}}$ & 0.631 & 0.686 & 0.695 & 0.697 & 0.719 & 0.624 & 0.692 & 0.714 & 0.703 & 0.656 & 0.651 & 0.633 & 0.633 & 0.612 & 0.685 & 0.678 & 0.673 & 0.616 & 0.664 \\
\hline & & & & & & & & & & & & & & 10 & 10. & & & & 8.40 \\
\hline $\mathrm{FeO}$ & 9.8 & & & & 8 & 9. & & & 7. & 1 & 1 & 1 & 1. & 1 & 1.12 & 0 & 0.97 & 1.23 & 1.00 \\
\hline & 1. & & 0 & 0 & 0 & 1.18 & 0.88 & 0. & 0. & 1.04 & 7.3 & 6.9 & 6.7 & 6.2 & 7.4 & 7.5 & 7.2 & 7.7 & 6.8 \\
\hline $\mathrm{CaO} / \mathrm{l}$ & 6.4 & 7. & 7. & 8 & 8. & 6.0 & 7.6 & 8. & 8. & 7.3 & 1.99 & 1.80 & 2.03 & 2.17 & 2.18 & 2.04 & 2.03 & 2.84 & 2.05 \\
\hline $\mathrm{Zr} / \mathrm{Y}$ & 1.86 & 2.15 & 2.13 & 2.05 & 2.09 & 2.18 & 2.11 & 2.04 & 2.07 & 1.96 & & & & & & & & & \\
\hline
\end{tabular}


Table 7. Trace element abundances (ppm) in selected samples from Hole 504B basalts.

\begin{tabular}{|c|c|c|c|c|c|c|c|c|c|c|c|c|c|c|c|c|c|c|}
\hline & $83-4^{a}$ & $83-6$ & $83-7$ & $83-10$ & $83-13$ & $83-18$ & 83-19 & $83-22$ & 83-32 & 83-35 & $83-40$ & $83-41$ & $83-52$ & $83-60$ & $83-67$ & $83-68$ & & \\
\hline & $\begin{array}{c}73-1 \\
126-130\end{array}$ & $\begin{array}{c}77-3 \\
11-13\end{array}$ & $\begin{array}{l}81-1 \\
64-68\end{array}$ & $\begin{array}{c}84-1 \\
77-79\end{array}$ & $\begin{array}{c}88-1 \\
109-113\end{array}$ & $\begin{array}{l}90-4 \\
49-53\end{array}$ & $\begin{array}{c}9-1 \\
127-132\end{array}$ & $\begin{array}{l}93-3 \\
27-32\end{array}$ & $\begin{array}{l}104-2 \\
24-27\end{array}$ & $\begin{array}{l}109-1 \\
49-52\end{array}$ & $\begin{array}{c}113-1, \\
129-133\end{array}$ & $\begin{array}{l}116-1, \\
43-46\end{array}$ & $\begin{array}{l}129-1 \\
76-81\end{array}$ & $\begin{array}{c}133-1, \\
101-106\end{array}$ & $\begin{array}{l}140-1 \\
40-42\end{array}$ & $\begin{array}{l}141-1 \\
16-20\end{array}$ & BCR-1 ${ }^{b}$ & $N^{c}$ \\
\hline $\mathrm{La}$ & 1.08 & 1.49 & 1.20 & 1.30 & 3.22 & 1.66 & 1.03 & 3.09 & 1.31 & 1.31 & 1.27 & 1.59 & 1.09 & 1.25 & 1.70 & 2.22 & $24.53(0.48)$ & 0.33 \\
\hline $\mathrm{Ce}$ & 5.2 & 5.4 & 4.8 & 5.1 & 9.3 & 6.0 & 3.9 & 12.3 & 5.5 & 4.9 & 4.0 & 5.4 & 4.1 & 4.3 & 6.2 & 6.1 & $53.7(0.7)$ & 0.88 \\
\hline Sm & 1.87 & 2.41 & 1.92 & 1.95 & 2.38 & 2.06 & 1.62 & $3.67^{\circ}$ & 2.09 & 1.97 & 1.73 & 2.30 & 1.57 & 2.13 & 2.47 & 1.80 & $6.73(0.09)$ & 0.181 \\
\hline Eu & 0.73 & 0.95 & 1.00 & 0.78 & 0.84 & 1.02 & 0.67 & 1.19 & 0.94 & 0.74 & 0.67 & 0.88 & 0.71 & 0.80 & 1.14 & 0.79 & $1.92(0.05)$ & 0.069 \\
\hline $\mathrm{Tb}$ & 0.55 & 0.78 & 0.67 & 0.58 & 0.69 & 0.55 & 0.50 & 0.9 & 0.7 & 0.64 & 0.56 & 0.73 & 0.49 & 0.71 & 0.8 & 0.52 & $1.09(0.09)$ & 0.047 \\
\hline $\mathrm{Yb}$ & 2.16 & 3.2 & 2.4 & 2.4 & 2.6 & 2.1 & 2.23 & 3.6 & 2.9 & 2.7 & 2.3 & 2.8 & 1.9 & 2.8 & 3.1 & 2.0 & $3.3(0.15)$ & 0.2 \\
\hline Lu & 0.34 & 0.52 & 0.42 & 0.41 & 0.42 & 0.37 & 0.39 & 0.55 & 0.44 & 0.42 & 0.39 & 0.48 & 0.31 & 0.46 & 0.51 & 0.38 & $0.51(0.02)$ & 0.034 \\
\hline Co & 58.3 & 59.0 & 52.5 & 59.7 & 49.2 & 50.0 & 53.8 & 49.9 & 50.6 & 51.5 & 58.3 & 59.5 & 116.8 & 66.0 & 74.1 & 54.8 & $35.4(0.4)$ & \\
\hline $\mathrm{Sc}$ & 42.9 & 48.1 & 40.7 & 41.7 & 42.6 & 40.8 & 40.9 & 38.5 & 40.1 & 42.7 & 40.6 & 46.1 & 36.8 & 34.9 & 44.6 & 40.6 & $31.9(0.4)$ & \\
\hline $\mathrm{Hf}$ & 1.3 & 2.0 & 1.4 & 1.3 & 2.0 & 1.9 & 1.0 & 3.1 & 1.7 & 1.3 & 1.1 & 1.7 & 0.9 & 1.5 & 1.6 & 1.3 & $5.1(0.4)$ & \\
\hline $\mathrm{Ta}$ & 0.3 & 0.4 & 0.4 & 0.4 & 0.5 & 0.5 & 0.2 & 0.5 & 0.4 & - & 0.5 & 0.3 & 0.4 & 0.5 & 0.9 & 0.6 & $0.9(0.2)$ & \\
\hline $\mathrm{La} / \mathrm{Yb}$ & 0.30 & 0.28 & 0.30 & 0.33 & 0.75 & 0.47 & 0.28 & 0.52 & 0.27 & 0.30 & 0.33 & 0.34 & 0.34 & 0.27 & 0.33 & 0.67 & & \\
\hline $\mathrm{La} / \mathrm{Sm}$ & 0.32 & 0.34 & 0.34 & 0.36 & 0.74 & 0.44 & 0.35 & 0.46 & 0.34 & 0.36 & 0.40 & 0.38 & 0.38 & 0.32 & 0.38 & 0.67 & & \\
\hline
\end{tabular}

a Sample identification number.

b $1 \sigma$ for standard BCR-1 analyzed as an unknown given in parentheses.

c $N$ refers to values used in chondrite-normalizing REE abundances. (Haskin et al., 1968)

Table 8. Average compositions of Hole 504B basalts.

\begin{tabular}{|c|c|c|c|c|c|}
\hline & $\begin{array}{l}\text { Legs } 69,70 \\
\text { Group } M^{\mathrm{a}} \\
\text { (4) }\end{array}$ & $\begin{array}{c}\text { Legs } 69,70 \\
\text { Group D } \\
(61)\end{array}$ & $\begin{array}{l}\text { Leg } 83 \\
\text { Group D' } \\
(56)\end{array}$ & $\begin{array}{l}\text { Leg } 83 \\
\text { Group T } \\
\text { (2) }\end{array}$ & $\begin{array}{l}\text { Leg } 83 \\
\text { Group I } \\
\text { (1) }\end{array}$ \\
\hline $\mathrm{SiO}_{2}$ & $50.4(1)$ & $50.4(5)$ & $49.9(6)$ & $50.6(14)$ & 48.7 \\
\hline $\mathrm{TiO}_{2}$ & 1.37 (1) & $0.92(9)$ & 0.92 (11) & $1.09(1)$ & 1.58 \\
\hline $\mathrm{Al}_{2} \mathrm{O}_{3}$ & 15.3 (1) & $15.9(7)$ & 15.5 (7) & $15.3(2)$ & 16.0 \\
\hline $\mathrm{Fe}_{2} \mathrm{O}_{3}{ }^{*}$ & 9.95 (14) & $9.87(70)$ & $10.1(7)$ & $9.86(15)$ & 10.71 \\
\hline $\mathrm{MnO}^{-}$ & 0.18 (1) & $0.16(2)$ & 0.19 (3) & $0.25(1)$ & 0.21 \\
\hline $\mathrm{MgO}$ & 8.17 (13) & $8.4(6)$ & $8.6(5)$ & $8.71(17)$ & 8.66 \\
\hline $\mathrm{CaO}$ & $12.5(2)$ & $12.5(7)$ & $13.0(3)$ & $12.2(7)$ & 11.88 \\
\hline $\mathrm{Na}_{2} \mathrm{O}$ & 2.5 (1) & 2.0 (1) & & $2.15(3)$ & 2.34 \\
\hline $\mathrm{K}_{2} \mathrm{O}$ & 0.06 (1) & $0.11(8)$ & $0.02(2)$ & $0.03(3)$ & .0 .01 \\
\hline $\mathrm{P}_{2}^{2} \mathrm{O}_{5}$ & 0.13 (1) & 0.07 (2) & 0.09 (1) & $0.11(3)$ & 0.19 \\
\hline$R \bar{b}$ & $<0.5$ & $1.3(16)$ & $<0.5$ & $<0.5$ & $<0.5$ \\
\hline $\mathrm{Sr}$ & $105(1)$ & 64 (9) & $55(7)$ & $73(2)$ & 75.6 \\
\hline Y & $31.2(3)$ & $23.3(24)$ & $22.8(28)$ & $23.5(10)$ & 23.9 \\
\hline $\mathrm{Ga}$ & $16.2(3)$ & $15.4)(9)$ & $15.2(7)$ & 14.8 (4) & 16.4 \\
\hline $\mathrm{Zr}$ & $103.7(4)$ & $47(5)$ & $46(6)$ & $67(1)$ & 131.9 \\
\hline $\mathrm{Nb}$ & 2.4 (2) & $0.6(3)$ & $0.9(2)$ & $4.2(3)$ & 1.6 \\
\hline $\mathrm{Zn}$ & 79 (2) & $77(8)$ & 80 (13) & 107 (4) & 89 \\
\hline $\mathrm{Ni}$ & 97 (2) & $117(25)$ & $117(34)$ & 107 (2) & 134 \\
\hline $\mathrm{Cr}$ & 257 (13) & 359 (74) & $336(87)$ & $342(16)$ & 287 \\
\hline v & 310 (12) & $274(31)$ & $263(34)$ & 257 (1) & 236 \\
\hline $\mathrm{LaN}^{\mathrm{b}}$ & & & $4.2(10)$ & 9.8 & 9.4 \\
\hline $\mathrm{Sm}_{\mathrm{N}}$ & & & $11.0(15)$ & 13.2 & 20.3 \\
\hline $\mathrm{Yb}_{\mathrm{N}}$ & & & $12.6(21)$ & 13.0 & 18.2 \\
\hline $\mathrm{Mg}^{\prime}$ & 0.644 (3) & & 0.653 (3) & 0.661 & 0.640 \\
\hline $\mathrm{Zr} / \mathrm{Nb}$ & 43 & 78 & 51 & 16 & 82 \\
\hline $\mathrm{Zr} / \mathrm{Y}$ & 3.32 & 2.02 & 2.02 & 2.85 & 4.00 \\
\hline $\mathrm{La} / \mathrm{Sm}_{\mathrm{N}}$ & & & 0.38 & 0.74 & 0.46 \\
\hline
\end{tabular}

Note: $N$ for each group in parentheses in the column heads.

a Basalt groups discussed in text and by Autio and Rhodes (1983). Values for basalts from Legs 69 and 70 are taken from Hole 504B data of Autio and Rhodes (1983).

b Normalizing values for REE are in Table 7. Values are given as mean and 1 S.D. (in parentheses, expressed in terms of last digit of the mean).

fied melt inclusions $5-50 \mu \mathrm{m}$ in size that are distributed throughout the core of the crystal. The core is mantled by an inclusion-free rim (Fig. 7A). Compositional zonation relative to the inclusion boundaries could not be detected. The second xenocryst type has an unresorbed core surrounded by an intermediate resorbed zone and is enclosed by an inclusion-free rim (Fig. 7B). The xenocrysts are compositionally zoned adjacent to the boundaries of the vermicular inclusions. In each xenocryst type the inclusion-free rim is compositionally like the groundmass feldspar.

Figure 2 and Table 3 present representative analyses of phenocryst and xenocryst plagioclase; groundmass plagioclase compositions are summarized in Figure 2. Traverses during analyses were chosen to pass along the direction in the crystal in which the most complete zoning history is recorded. Hole 504B (leg 83) plagioclase compositions are distinctive in their relatively high An content, up to $90 \mathrm{~mol} \%$. Anorthite contents of as much as $94 \mathrm{~mol} \%$ are reported by Natland et al. (1983) for plagioclase in the upper portion of Hole 504B. Potassium is extremely low (less than 0.05 wt. \% in all Leg 83 feldspars. Xenocryst core compositions may be either more or less calcic than the coexisting phenocrysts of the same unit (Fig. 2). In many phenocrysts and xenocrysts the highest An content does not occur in the core of the crystals, but rather at some intermediate position between core and rim.

The backscattered electron image (BEI) in Figure 8A shows a feldspar xenocryst of composition $\mathrm{An}_{70}$ zoned to a more calcic composition (brighter in BEI) like that of the groundmass and phenocryst-rim composition in a zone adjacent to the devitrified melt inclusions. In Figures $8 \mathrm{~B}, \mathrm{C}$ the opposite situation is exemplified. The zone adjacent to the cavities or inclusions is lower in calcium (darker in BEI), while the coexisting phenocryst shows a narrow zone or more anorthitic plagioclase.

Figure 9E shows the relationship between mol\% An and $\mathrm{Mg} /(\mathrm{Mg}+\mathrm{Fe})$ in plagioclase phenocrysts, xenocrysts, and groundmass crystals. The correlation between An and $\mathrm{Mg} /(\mathrm{Mg}+\mathrm{Fe})$ is positive for anorthite contents less than $79 \mathrm{~mol} \%$, but scattered for more calcic feldspars. The maximum $\mathrm{Mg} /(\mathrm{Mg}+\mathrm{Fe})$ in Leg 83 plagioclase corresponds to the maximum whole-rock $\mathrm{mg}$ value in Leg 83 basalts $(0.72$ in Sample 504B-129-1, 76-81 cm), and the anorthite content at this maximum is closely approximated by the calculated (Drake, 1976) maximum An content in equilibrium with these basalts. A similar relationship is observed for plagioclases in North Atlantic basalts, DSDP Leg 11 (Ayuso et al., 1976) and DSDP Legs 45 and 46 (Kuo and Kirkpatrick, 1982) except that the plagioclase $\mathrm{Mg} /(\mathrm{Mg}+\mathrm{Fe})$ maxima occur at lower anorthite contents. This is consistent with the unusually high concentration of $\mathrm{CaO}$ in Hole 504B basalts.

Figures 9A-D compare representative traverses across coexisting phenocrysts and xenocrysts. As shown in 


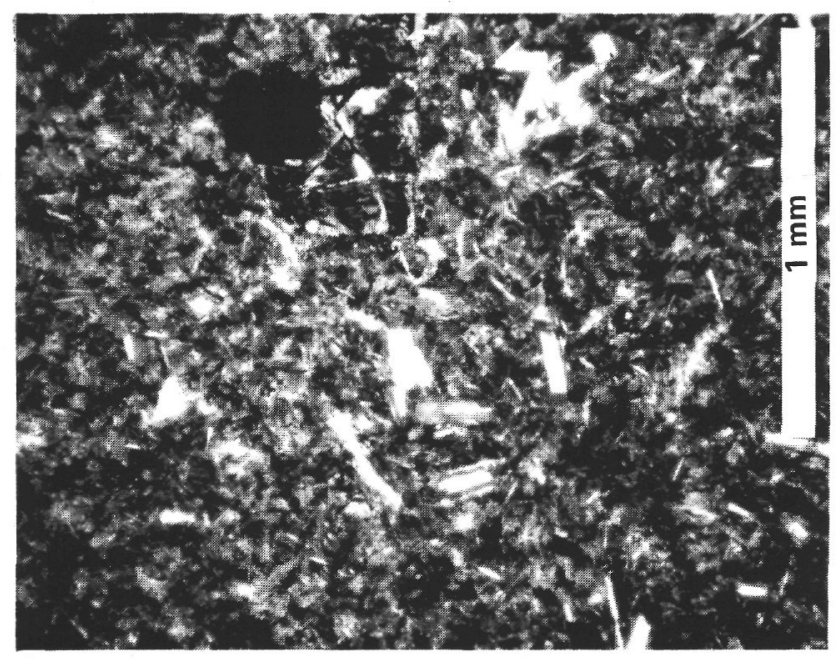

A

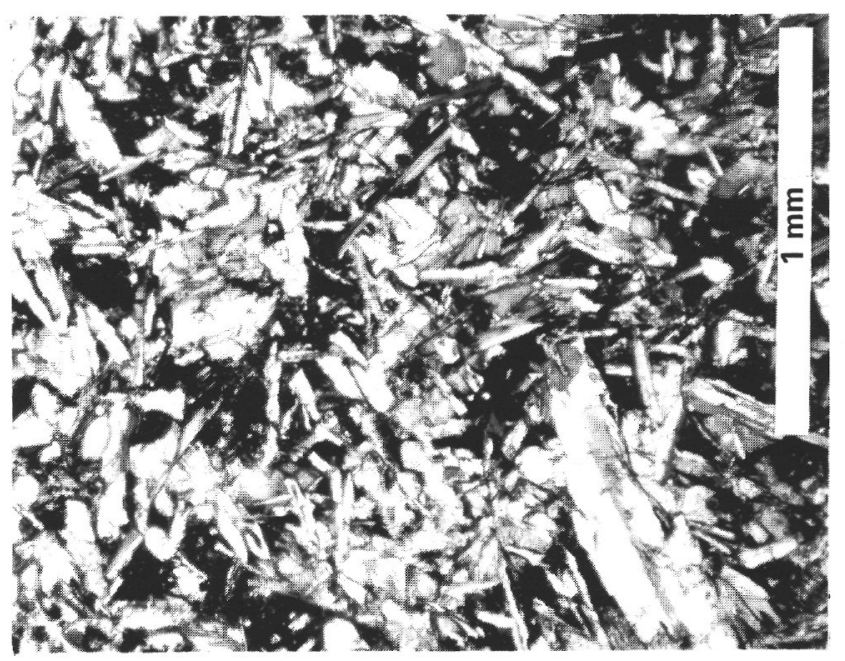

B

Figure 1. Photomicrographs of aphyric basalts. (A) Fine-grained intergranular texture; Sample 504B-93-3, 27-32 cm, Group M' basalt. Crossed polars. (B) Medium-grained subophitic textures; Sample 504B-107-2, 71-75 cm, Group D' basalt. Crossed polars.

Figure 2, xenocryst core compositions may be either more or less calcic than the coexisting phenocryst cores. $\mathrm{The} \mathrm{Mg} /(\mathrm{Mg}+\mathrm{Fe})$ ratios in plagioclase xenocryst cores are lower than those for the associated phenocrysts. Furthermore, although the mg value decreases systematically with decreasing An content for anorthite contents less than $70 \mathrm{~mol} \%$, for more calcic compositions, the variation between $\mathrm{Mg} /(\mathrm{Mg}+\mathrm{Fe})$ and mol\% An is more complex. The spectrum of zoning trends observed within and among samples indicates that a single mixing event is inadequate to explain the compositional variation in the plagioclase phenocrysts and xenocrysts in basalts from Hole 504B.

Olivine is the second most abundant phenocryst phase, constituting up to $2 \%$ of the rock. However, with the exception of Sample 504B-73-1, 126-130 cm, olivine is totally altered to one or more phyllosilicate minerals in all Leg 83 basalts. Pseudomorphs display euhedral to subhedral morphologies and range from 0.1 to $6 \mathrm{~mm}$ in size. Microprobe analyses of olivine in Sample 504B-73-1, 126$130 \mathrm{~cm}$ indicate a compositional range from $\mathrm{Fo}_{85}$ to $\mathrm{Fo}_{87}$. This is consistent with the olivine compositions reported by Natland et al. (1983) for the upper portion of Hole 504B. Calculations using the equilibrium distribution coefficient (Roeder and Emslie, 1970)

$$
K_{D}=\frac{\mathrm{X}_{\mathrm{FeO}}^{\mathrm{OI}} \mathrm{X}_{\mathrm{MgO}}^{\mathrm{liq}}}{\mathrm{X}_{\mathrm{MgO}}^{\mathrm{Ol}} \mathrm{X}_{\mathrm{FeO}}^{\mathrm{liq}}}=0.33
$$

indicate that this olivine composition is in equilibrium with the bulk rock composition in which it occurs.

Clinopyroxene, although abundant in the groundmass, is less common as a phenocryst phase. When present it forms large crystals up to $6 \mathrm{~mm}$ long. Crystal morphologies range from anhedral to euhedral and are often twinned on (100). In samples where the groundmass is medium grained, the clinopyroxene phenocrysts are strongly zoned with respect to $\mathrm{FeO}$ (up to $22.29 \mathrm{wt} . \%$, Fig. 4A). $\mathrm{Cr}_{2} \mathrm{O}_{3}$ is commonly less than 0.3 wt. $\%$, but the interiors of some phenocrysts have up to $1.33 \mathrm{wt} . \%$.
These high $\mathrm{Cr}$ cores appear compositionally like the resorbed crystals identified as xenocrysts in the aphyric basalts.

Clinopyroxene xenocrysts, defined primarily on the basis of their rounded or resorbed morphologies, are present in phyric basalts. They are analogous to those described for the clinopyroxene xenocrysts in the aphyric basalts and occur as solitary xenocrysts or with plagioclase in clusters that are interpreted as basaltic xenoliths. These xenolithic aggregates are similar in texture and grain size to many of the massive aphyric basalts. In common with the clinopyroxene xenocrysts in aphyric basalts, clinopyroxene xenocrysts in phyric basalts are generally high in $\mathrm{Cr}$ (Table 1).

Chromian spinel has been observed in all petrographic groups, but it occurs predominantly in two distinct associations in basalts from Hole 504B: (1) as goldenbrown crystals included in plagioclase and olivine (one rare occurrence in clinopyroxene) in fine- to mediumgrained basalts and (2) as discrete, reddish brown crystals in dike and pillow chilled margins. In general, spinels are more common and occur in greater abundance in the latter group. Of the 32 samples in which $\mathrm{Cr}$ spinels are observed, only 9 are medium-grained aphyric or phyric basalts. In these coarser-grained lavas, one to three grains 10 to $200 \mu \mathrm{m}$ in size are present in a standard thin section. In dike or pillow chilled margins, 5 to 10 times this number of spinels occur. Chromian spinels are typically euhedral to subhedral, but embayed or rounded morphologies are observed in some chilled margins.

These two physical associations describe two overlapping chemical populations (Table 4). Figure 10 is a diagram of $\mathrm{Cr} /(\mathrm{Cr}+\mathrm{Al})$ vs. $\mathrm{Mg} /\left(\mathrm{Mg}+\mathrm{Fe}^{2+}\right)$ in spinel. Those spinels partially or totally enclosed in olivine or plagioclase (symbolized by the filled circles) are generally higher in $\mathrm{Cr} /(\mathrm{Cr}+\mathrm{Al})$ and lower in $\mathrm{Mg} /(\mathrm{Mg}+$ $\left.\mathrm{Fe}^{2+}\right)$; they fall in the compositional field designated by Sigurdsson and Schilling (1976) as magnesiochromites: $\mathrm{Cr}$ is the dominant trivalent cation and $\mathrm{Mg}^{2+}>\mathrm{Fe}^{2+}$. These spinels exhibit zoning trends in which $\mathrm{Fe}^{3+}$ and 

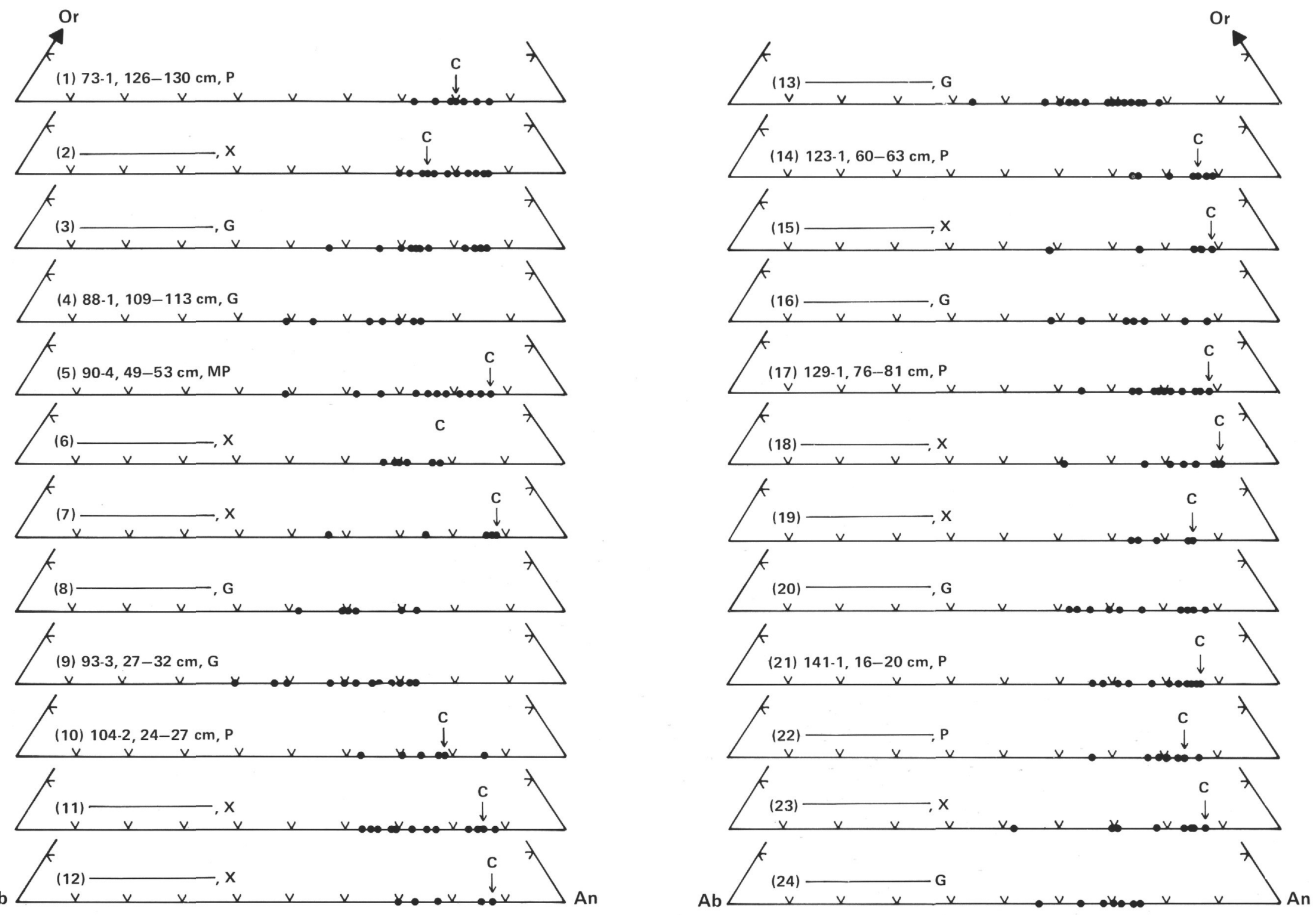

Figure 2. Microprobe analyses of phenocryst, xenocryst, and groundmass plagioclase. $\mathrm{P}=$ phenocryst, $\mathrm{MP}=$ microphenocryst, $\mathrm{X}=\mathrm{xenocryst}$, and $\mathrm{G}=$ groundmass. $\mathrm{C}$ indicates the core composition of the crystal. Solid line denotes analysis from the same sample as those in the preceding ternary plot. 


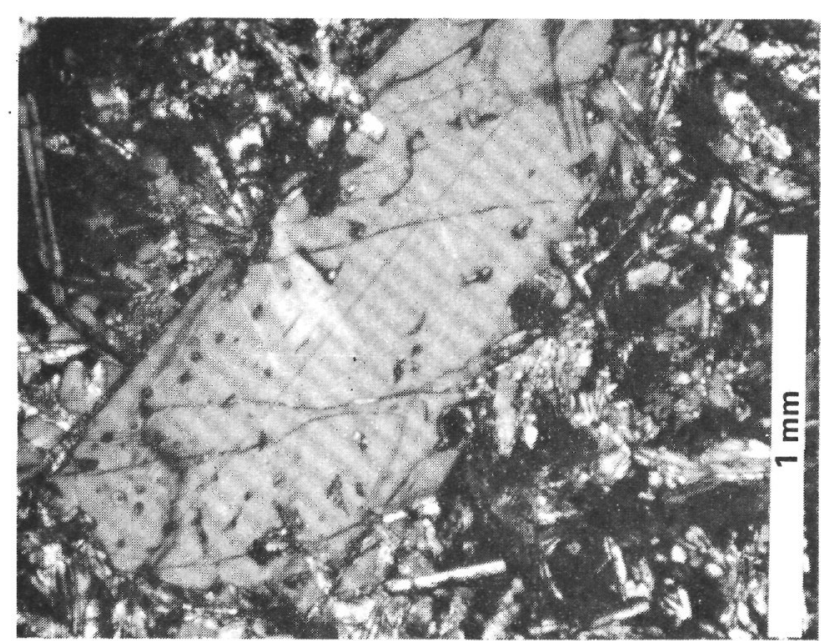

A

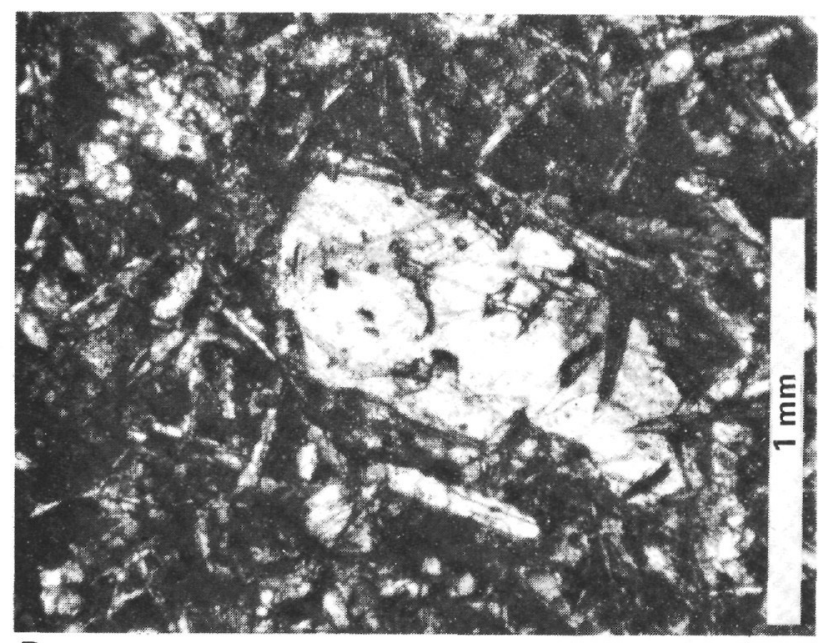

B

Figure 3. Photomicrographs of disequilibrium clinopyroxene phenocrysts. Note subophitic textures (partially enclosing groundmass plagioclase) of clinopyroxene rims. (A) Sample 504B-88-1, 109-113 cm. Crossed polars. (B) Sample 504B-1 16-1, 43-46 cm. Crossed polars.

$\mathrm{Cr}$ increase and $\mathrm{Al}$ decreases toward the rim. In contrast, spinels occurring in chilled margins (filled triangles) tend to be lower in $\mathrm{Cr} /(\mathrm{Cr}+\mathrm{Al})$, but higher in $\mathrm{Mg} /\left(\mathrm{Mg}+\mathrm{Fe}^{2+}\right)$ and fall in the field referred to as chromian spinels $\left(\mathrm{Al}>\mathrm{Cr}, \mathrm{Mg}^{2+}>\mathrm{Fe}^{2+}\right)$. Zoning trends in these spinels exhibit increasing $\mathrm{Fe}^{3+}$ and $\mathrm{Al}$, but decreasing $\mathrm{Cr}$ from core to rim. Spinel compositions from the Mid-Atlantic Ridge (Dungan and Rhodes, 1978; Sigurdsson and Schilling, 1976) (open circles) are plotted for reference. Also plotted are average zoning trends (arrows) observed in three samples from the Siqueiros Fracture Zone (at $8^{\circ} 30 \mathrm{~N}$ near its intersection with the crest of the East Pacific Rise; Natland et al., 1983). From left to right, the arrows correspond to spinels that crystallized from liquids with increasing $\mathrm{Mg} /\left(\mathrm{Mg}+\mathrm{Fe}^{2+}\right)$ ( 0.71 for Curve 1 and 0.65 for Curve 3 ). In accord with the high $\mathrm{mg}$ values of basalts from Hole 504B, spinels in these lavas are generally higher in $\mathrm{Mg} /\left(\mathrm{Mg}+\mathrm{Fe}^{2+}\right)$ than basalts from the Mid-Atlantic Ridge or the East Pacific Rise throughout the range of $\mathrm{Cr} /(\mathrm{Cr}+\mathrm{Al})$.

\section{BASALT CHEMISTRY}

The Leg 83 basalts occur as three distinct chemical groups, designated $\mathrm{D}^{\prime}, \mathrm{M}^{\prime}$, and $\mathrm{T}$, in order to conform with the nomenclature of Autio and Rhodes (1983) for the upper part of Hole 504B. The majority of basalts belong to Group $\mathrm{D}^{\prime}$, whereas Groups $\mathrm{M}^{\prime}$ and $\mathrm{T}$ are comparatively rare.

The single analyzed sample of Group $\mathbf{M}^{\prime}$ basalt (Sample 504B-93-3, 27-32 cm) occurred at a depth of $1021.5 \mathrm{~m}$ below the seafloor (BSF). It has a moderate $\mathrm{mg}$ value (0.64), $\mathrm{TiO}_{2}$ (1.58 wt.\%), $\mathrm{Na}_{2} \mathrm{O}(2.34 \%), \mathrm{Y}(33 \mathrm{ppm})$, $\mathrm{Zr}(132 \mathrm{ppm}), \mathrm{Nb}(1.4 \mathrm{ppm}), \mathrm{Hf}(3.5 \mathrm{ppm}), \mathrm{La}(9.4 \times$ chondrite), and $\mathrm{Sm}(20.3 \times$ chondrite $)$ abundances. These values are typical of Type I MORB (Bryan et al., 1976) and resemble the Group $M$ basalts (Autio and Rhodes, 1983) at higher stratigraphic levels of Hole 504B. A substantial fractionation history involving olivine, plagioclase, and spinel is indicated by the low abundances of $\mathrm{Cr}(287 \mathrm{ppm})$ and $\mathrm{Ni}(134 \mathrm{ppm})$, and by moderate $\mathrm{MgO}(8.7 \%)$, $\mathrm{FeO}^{*}(9.6 \%)$, and $\mathrm{CaO}(11.9 \%)$ abundances.

Most Leg 83 basalts (Group D') are highly depleted in all magmaphile elements relative to normal Type I and II MORB (Bryan et al., 1976). They have extremely low abundances of $\mathrm{Zr}$ (36-56 ppm), Y (17-28 ppm), $\mathrm{Nb}(<0.5-1.3 \mathrm{ppm}), \mathrm{La}(3.2-5 \times$ chondrite $), \mathrm{Hf}(0.9-$ $1.9 \mathrm{ppm})$, and $\mathrm{Ta}(<0.5 \mathrm{ppm})$ with low $\mathrm{TiO}_{2}(0.71-$ $1.12 \%), \mathrm{Na}_{2} \mathrm{O}(1.5-2.5 \%)$, and $\mathrm{K}_{2} \mathrm{O}(<0.01-0.17 \%)$ concentrations. This group is very similar to the Group D basalts (Autio and Rhodes, 1983) from the upper (Legs 69 and 70) portion of Hole 504B.

One-atmosphere melting experiments on comparable basalts from higher stratigraphic levels in Hole 504B (Autio and Rhodes, 1981, 1983) can be used to infer that the Group $\mathrm{D}^{\prime}$ basalts are also generally saturated in olivine and plagioclase, and near clinopyroxene saturation. Consequently, although these basalts have low incompatible element abundances, they are not primitive and have undergone substantial magmatic evolution. Both chemical and petrographic data support this conclusion. Abundances of $\mathrm{MgO}$ (7.7-10.\%), $\mathrm{FeO}^{*}$ (7.95-10.46\%), $\mathrm{Ni}$ (76-246 pm), $\mathrm{Cr}$ (168-532 ppm), and mg values (0.590.72 ) all imply a substantial fractionation history. A few samples, such as Sample 504B-129-1, 76-81 cm, are presumed to be more primitive with only olivine on the liquidus because of their higher $\mathrm{MgO}$ (10.7\%), Ni (246 $\mathrm{ppm})$, and $\mathrm{Cr}(532 \mathrm{ppm})$ abundances and $\mathrm{mg}$ value $(0.72)$. Although this is similar to some proposed primary ocean-floor basaltic compositions (Frey et al., 1974; Rhodes et al., 1978; Basaltic Volcanism Study Project, 1981), reasons for believing that Sample 504B-129-1, $76-81 \mathrm{~cm}$ is not primary and has evolved significantly will be presented in the discussion.

Two samples (Samples 504B-90-4, 49-53 cm; 504B$141-1,16-20 \mathrm{~cm}$ ) have anomalously high $\mathrm{La} / \mathrm{Yb}$ values ( 0.47 and 0.67 , respectively) compared with other samples from this group $(\mathrm{La} / \mathrm{Yb}=0.27-0.34)$. Sample 504B-140-1, 40-42 cm has an anomalously high $\mathrm{Zr} / \mathrm{Y}$ value of 2.84. However, these samples are comparable to the rest of Group $\mathrm{D}^{\prime}$ in all other elemental ratios and abundances. 

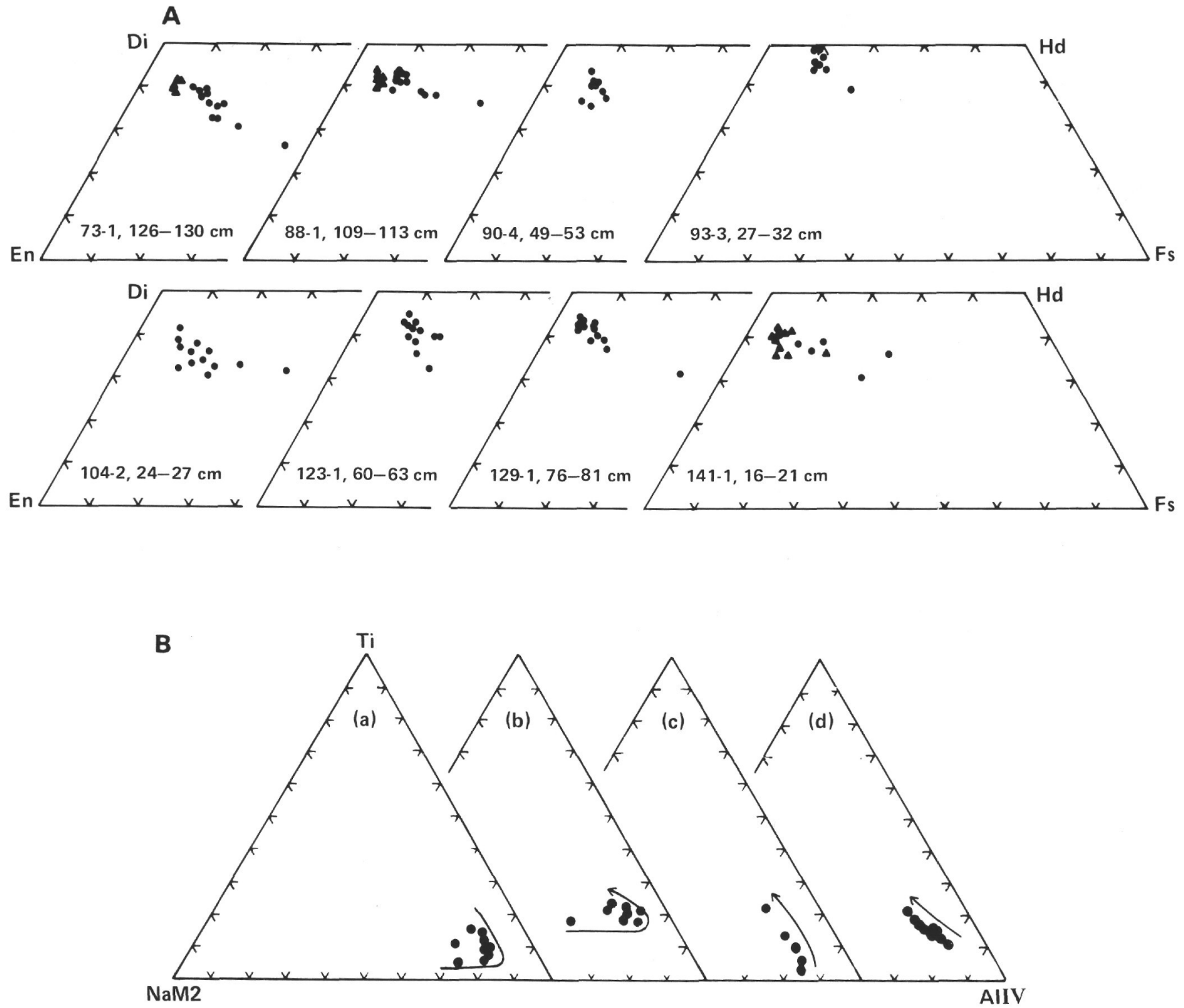

Figure 4. (A) Clinopyroxene phenocryst, xenocryst, and groundmass compositions represented in terms of quadrilateral components, Di-Hd-En-Fs. Xenocryst, $\mathbf{\Delta}$; phenocrysts and groundmass, $\boldsymbol{\bullet}$. Xenocrysts have consistently more $\mathrm{Mg}$ - and Ca-rich compositions than coexisting phenocryst or groundmass clinopyroxenes. Clinopyroxenes in Group $\mathrm{M}^{\prime}$ basalt, Sample 504B-93-3, 27-32 cm, have distinctly higher Ca content than clinopyroxenes from all other groups. (B) Representative trends in clinopyroxene "others" components, NaM2, AlIV, and Ti: (a) xenocryst in aphyric basalt Sample 504B-90-4, 49-53 cm (enriched Group D'); (b) xenocryst in aphyric basalt Sample 504B-93-3, 27-32 cm (Group M'); (c) phenocryst in phyric basalt Sample 504B-129-1, 76-81 cm (Group D'); (d) phenocryst in phyric basalt Sample 504b-141-1, 16-20 cm (enriched Group $\mathrm{D}^{\prime}$ ). Arrows indicate generalized zoning trend from core to rim. Notice the early enrichment in NaM2 followed by an increase in AlIV prior to Ti enrichment in clinopyroxene xenocrysts. Clinopyroxene phenocrysts and groundmass crystals show only the Ti enrichment from core to rim.

The Group T basalts (Samples 504B-88-1, 109-113 cm; 504B-91-4, 39-43 cm) occur in two thin layers. These samples have low abundances of most moderately incompatible elements: $\mathrm{TiO}_{2}(1.08-1.10 \%), \mathrm{Na}_{2} \mathrm{O}(1.9-$ $2.1 \%)$, Y (23-24 ppm), Hf (2 ppm), and Zr (66.6-68.2 ppm). However, highly incompatible elements such as $\mathrm{La}(9.8 \times$ chondrite $)$ and $\mathrm{Nb}(3.8-4.2 \mathrm{ppm})$ have been greatly enriched relative to the other magmaphile elements. mg values (0.66) and abundances of $\mathrm{Cr}$ (330-353 ppm), Ni (105-108 ppm), $\mathrm{MgO}(8.6-8.8 \%)$, and $\mathrm{FeO}^{*}$ $(8.8-9.0 \%)$ indicate that this group is not primitive, having olivine and plagioclase on the liquidus, and possibly clinopyroxene. Several samples from the upper por- tion of Hole 504B (Cores 18 and 19, Marsh et al., 1983; Etoubleau et al., 1983) are even more enriched in $\mathrm{Nb}$ (9$13 \mathrm{ppm})$ relative to the other magmaphile elements $(\mathrm{Zr}$ $=85-98 \mathrm{ppm} ; \mathrm{Y}=26-32 \mathrm{ppm}$ ).

Major, trace, and rare earth element data for Hole 504B basalts are summarized in Figures 11-18. Data used to construct the "MORB fields" on all figures for this section have been obtained using similar analytical techniques to those used in this study. Data from DSDP Legs 2, 3, 9, 17, 34, 45, 49, and Juan de Fuca dredge hauls (Frey et al., 1974; Bass et al., 1975; Rhodes et al., 1978; Tarney et al., 1979; Rhodes, unpublished data; Liias and Rhodes, unpublished data) are included. Less than 


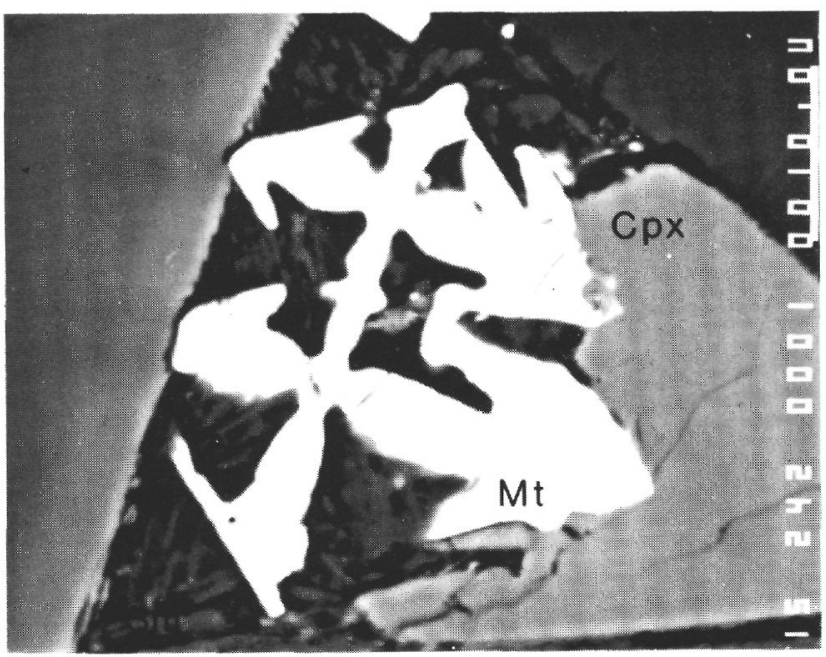

A

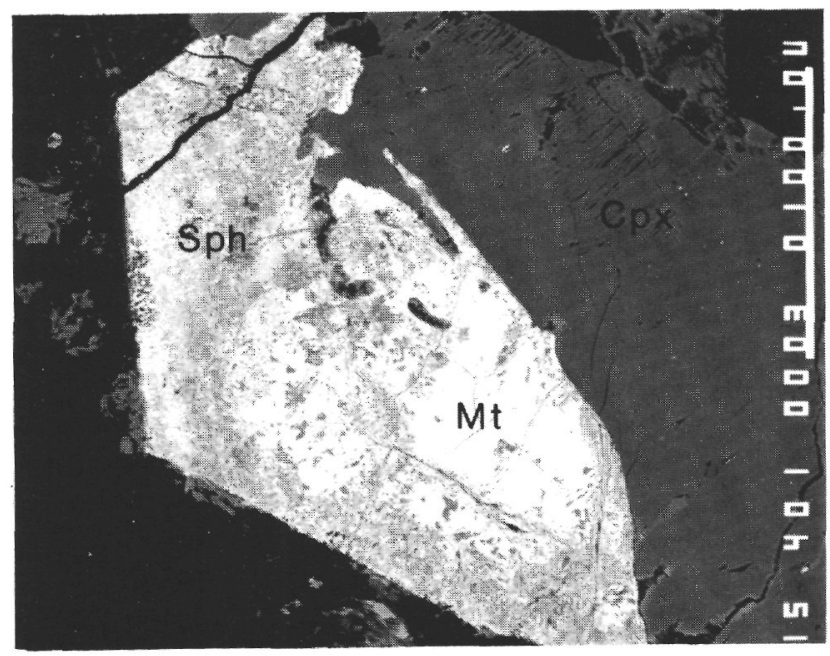

B

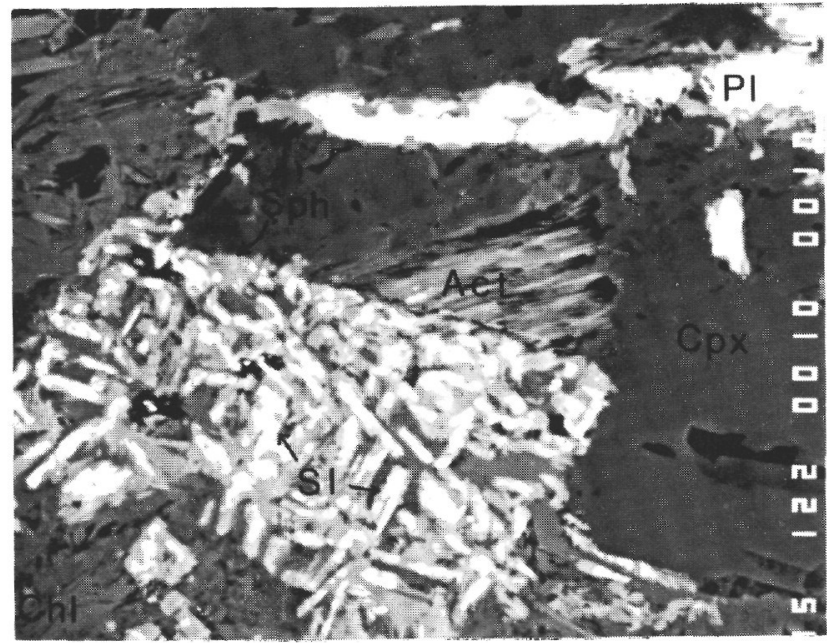

C

Figure 5. Backscattered electron images (BEI) of FeTi-oxides in Hole 504B basalts, Leg 83. (A) unaltered skeletal FeTi-oxide in Sample 504B-

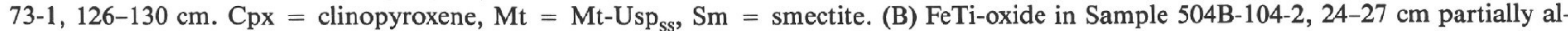
tered to sphene. $\mathrm{Sph}=$ sphene. $(\mathrm{C})$ FeTi-oxides in Sample 504B-93-3, 27-32 cm. Mt-Usp ss $_{\text {s }}$ completely altered and consists of an intergrowth of sphene plus ilmenite. Ilmenite is partially altered to sphene as well. PI = "primary" ilmenite, SI = "secondary" ilmenite, Chl $=$ chlorite, and Act $=$ actinolite. The sequence of numbers printed along the right margin in these photos and all subsequent BEI photographs correspond to the following information (from bottom to top): accelerating voltage (e.g., $15 \mathrm{kV}$ ); magnification (e.g., $242=24 \times$ $\left.10^{2}\right)$; photo sequence number $(001=$ first photo taken $)$; scale including scale bar and length in microns.

$3 \%$ of the data plots at $\mathrm{Zr}$ values less than $55 \mathrm{ppm}$ so the parts of the fields extending below $55 \mathrm{ppm} \mathrm{Zr}$ are less well defined. Several of the figures show reference lines of constant elemental ratios. Except as noted, open circles denote data from the upper part of Hole 504B (Autio and Rhodes, 1983); filled circles denote data from the lower part of Hole 504B (this chapter). Bulk rock chemistries for both upper and lower portions of Hole 504B are from the same laboratory under the same operating conditions and are directly comparable.

In Figures $11 \mathrm{~A}-\mathrm{D}\left(\mathrm{MgO}, \mathrm{CaO}, \mathrm{Al}_{2} \mathrm{O}_{3}\right.$, and $\mathrm{FeO}^{*} /$ $\mathrm{MgO}$ vs. $\mathrm{Zr}$ ) it can be seen that almost all Hole 504B basalts are highly depleted in $\mathrm{Zr}$ relative to other MORB. However, $\mathrm{MgO}$ and $\mathrm{FeO} * / \mathrm{MgO}$ values for the CRRZ basalts are similar to those of moderately evolved MORB. Both $\mathrm{CaO}$ and $\mathrm{Al}_{2} \mathrm{O}_{3}$ abundances are elevated in the CRRZ basalts relative to other MORB with comparable
$\mathrm{MgO}$ and $\mathrm{FeO}^{*} / \mathrm{MgO}$ values. Data for the upper and lower portions of Hole 504B are similar.

A plot of Ni vs. Zr (Fig. 12) indicates that most of Hole 504B basalts are lower in $\mathrm{Zr}$ for a similar Ni value (i.e., "state of evolution") than normal Type I and II MORB. A model fractionation trend of olivine, followed by plagioclase and then clinopyroxene, is shown for "normal" MORB. Most of the CRRZ basalts appear to follow a similar trend that is depleted in $\mathrm{Zr}$ relative to the normal trend.

In general, Type I MORB have $\mathrm{Zr} / \mathrm{Nb} \geq 30$, and Type II MORB have $\mathrm{Zr} / \mathrm{Nb} \leq 10$ (Erlank and Kable, 1976; Bryan et al., 1976; Basaltic Volcanism Study Project, 1981). Almost all Hole 504B basalts have $\mathrm{Zr} / \mathrm{Nb}$ ratios of $>40$. The only exceptions are the Group $\mathrm{T}$ basalts with $\mathrm{Zr} / \mathrm{Nb} \cong 16$ and the high $\mathrm{Nb}$ samples from Leg 69 that have $\mathrm{Zr} / \mathrm{Nb} \leq 10$ (Marsh et al., 1983; Etoubleau et 


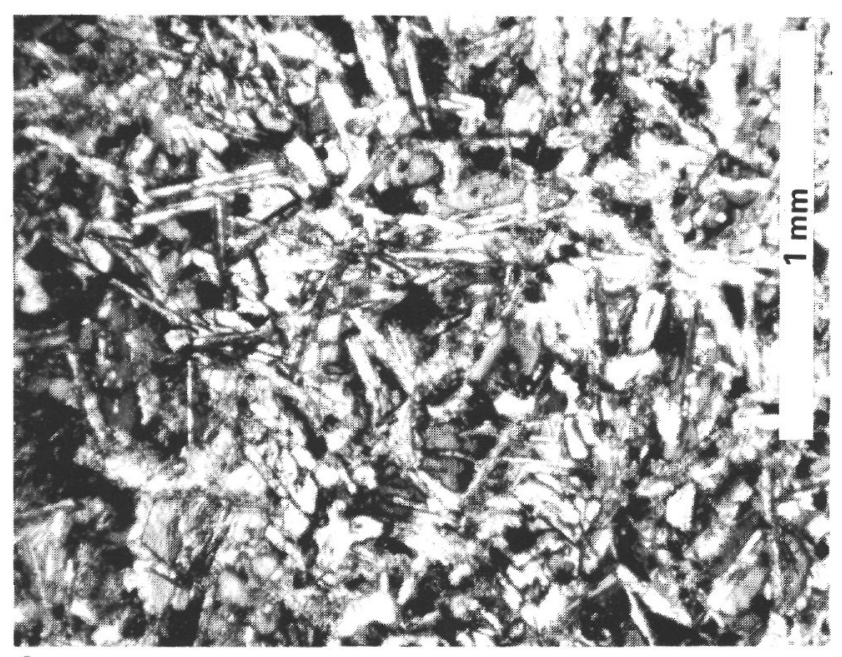

A

Figure 6. Photomicrographs of phyric basalts. (A) Fine-grained intersertal texture with plumose clinopyroxene; Sample 504B-76-1, 45-47 cm, OP basalt, Group D ${ }^{\prime}$. Crossed polars. (B) Medium-grained, subophitic texture, Sample 504B-141-1, 16-20 cm, OPC basalt, enriched Group $\mathrm{D}^{\prime}$. Crossed polars. al., 1983; Fig. 13). Although the abundances of $\mathrm{Zr}$ and $\mathrm{Nb}$ are strongly depleted in most CRRZ basalts, their $\mathrm{Zr} / \mathrm{Nb}$ ratios are similar to Type I MORB. The high $\mathrm{Nb}$ basalts from Leg 69 resemble Type II MORB, while Group $\mathrm{T}$ basalts fall in between. Group $\mathrm{T}$ basalts are similar to the anomalous Juan de Fuca basalts (Liias and Rhodes, 1982) and most of the basalts generated at the modern Costa Rica Rift (Autio and Rhodes, unpublished). Group $\mathrm{T}$ basalts cannot be derived by mixing Group $\mathrm{D}^{\prime}$ and $\mathbf{M}^{\prime}$ basalts.

The Group $\mathrm{D}^{\prime}$ and $\mathrm{D}$ basalts are all depleted in $\mathrm{TiO}_{2}$, $\mathrm{Y}$, and $\mathrm{Zr}$ relative to normal MORB (Figs. 14-16) at a similar level of magmatic evolution ( $\mathrm{MgO}, \mathrm{FeO}^{*} / \mathrm{MgO}$, $\mathrm{Ni}$, and $\mathrm{Cr}$ values). Additionally, they have lower $\mathrm{Zr} / \mathrm{TiO}_{2}$ (50) and $\mathrm{Zr} / \mathrm{Y}$ (2) and higher $\mathrm{Y} / \mathrm{TiO}_{2}$ (25) than normal MORB ( $\geq 65, \geq 3$, and $\leq 20$, respectively). There is a close coincidence of the data for the upper and lower portions of Hole 504B (Groups D and $\mathrm{D}^{\prime}$; Table 8). Groups $\mathrm{M}$ and $\mathrm{M}^{\prime}$ lie at higher $\mathrm{Zr}, \mathrm{Y}$, and $\mathrm{TiO}_{2}$ values and have more normal ratio of $\mathrm{Zr} / \mathrm{TiO}_{2}(75-80), \mathrm{Y} / \mathrm{TiO}_{2}$ $(\sim 22)$, and $\mathrm{Zr} / \mathrm{Y}$ (3.2-4). Group $\mathrm{T}$ basalts have a higher $\mathrm{Zr}$ for a given $\mathrm{Y}$ or $\mathrm{TiO}_{2}$ value, although their $\mathrm{Zr}$, $\mathrm{Y}$, and $\mathrm{TiO}_{2}$ abundances fall within the limits of the depleted basalt groups. Consequently, they exhibit higher $\mathrm{Zr}$ / $\mathrm{TiO}_{2}(60)$ and $\mathrm{Zr} / \mathrm{Y}(2.9)$ values. In Group $\mathrm{T}, \mathrm{Y}$ is also slightly depleted relative to $\mathrm{TiO}_{2}$, leading to lower (and more normal) $\mathrm{Y} / \mathrm{TiO}_{2}$ values $(\approx 22)$. The high $\mathrm{Nb}$ samples from the top of Hole 504B have similar ratios: $\mathrm{Zr}$ / $\mathrm{TiO}_{2}=70, \mathrm{Zr} / \mathrm{Y}=3$, and $\mathrm{Y} / \mathrm{TiO}_{2}=22$ (Marsh et al., 1983; Etoubleau et al., 1983).

Although the Group D and $\mathrm{D}^{\prime}$ basalts follow fairly coherent $\mathrm{TiO}_{2}$ vs. $\mathrm{Y}$ and $\mathrm{Zr}$ vs. $\mathrm{TiO}_{2}$ trends (Figs. 14, $15)$, there is scatter on these and the $\mathrm{Zr} / \mathrm{Y}$ vs. $\mathrm{Zr}$ plots (Fig. 16). A calculated fractionation path for Sample 504B-129-1, 76-81 cm (83-52) is shown in Figure 16, where the crystallization sequence was modeled as olivine, followed by olivine + plagioclase, followed by olivine + plagioclase + clinopyroxene. The length of the

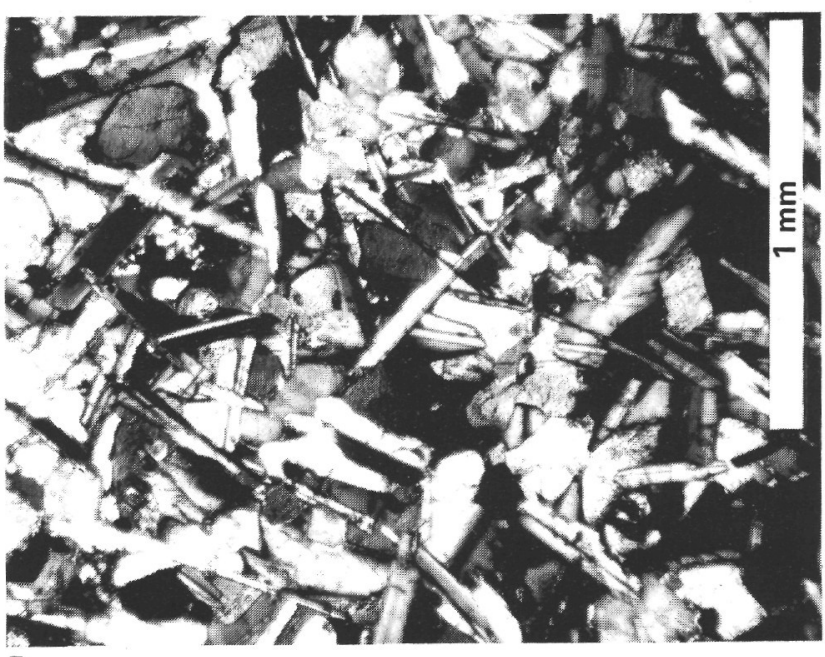

B path shows approximately $45 \%$ crystal fractionation of the original composition. It can be seen that more scatter exists than can be explained by simple fractionation, phenocryst addition, mixing of consanguineous magmas, or alteration. Nor can the depleted types be fractionated to produce the Group $\mathbf{M}, \mathbf{M}^{\prime}$, or T basalts.

Group $\mathrm{D}^{\prime}$ basalts are remarkably constant in their rare earth element composition (Figs. 17A, B, and 18). Consonant with their depletion in $\mathrm{Zr}$ and $\mathrm{Nb}$, Group $\mathrm{D}^{\prime}$ basalts are also markedly depleted in LREE $\left(\mathrm{La}_{\mathrm{N}} /\right.$ $\mathrm{Yb}_{\mathrm{N}}=0.27-0.34$ ) when compared with normal (Type I) MORB (0.72; Bryan et al., 1976). Chondrite-normalized $\mathrm{La} / \mathrm{Yb}$ for the active Galapagos Spreading Center ranges from 0.57 to 1.17 (Clague et al., 1981; Schilling et al., 1976). In contrast, Groups $\mathbf{M}^{\prime}$, T, and two unusual Group D' basalts (Sample 504B-88-1, 109-113 cm; 504B-91-4, 39-43 cm) are enriched in LREE relative to the majority of CRRZ basalts, but have $\mathrm{La}_{\mathrm{N}} / \mathrm{Yb}_{\mathrm{N}}$ values $(0.47-0.75)$ within the range observed for normal Type I MORB (Figs. 17C and 18). Although the variation in REE for most Group D' basalts can be modeled by fractional crystallization of low-pressure phenocryst phases, it has been shown that these phases do not significantly fractionate the REE relative to each other during crystallization in most oceanic basalts (Frey et al., 1974); the original shape of the chondrite-normalized rare earth pattern is maintained even though the concentration of the REE increases. On the other hand, lowpressure fractionation of Group $\mathrm{D}^{\prime}$ basalts cannot produce the compositions of Groups $\mathbf{M}^{\prime}$, $\mathbf{T}$, and the two unusual Group D' basalts. It is possible that a complex sequence of mixing and fractionation processes could produce the enriched Group $\mathrm{D}^{\prime}$ basalts from the "normal" Group D'. However, the distribution of $\mathrm{La}_{\mathrm{N}} / \mathrm{Yb}_{\mathrm{N}}$ vs. $\mathrm{Yb}_{\mathrm{N}}$ (Fig. 18) indicates that mixing of enriched and depleted magmas is not responsible for the remaining spectrum of compositions observed in Hole 504B. This will be discussed in more detail later. 


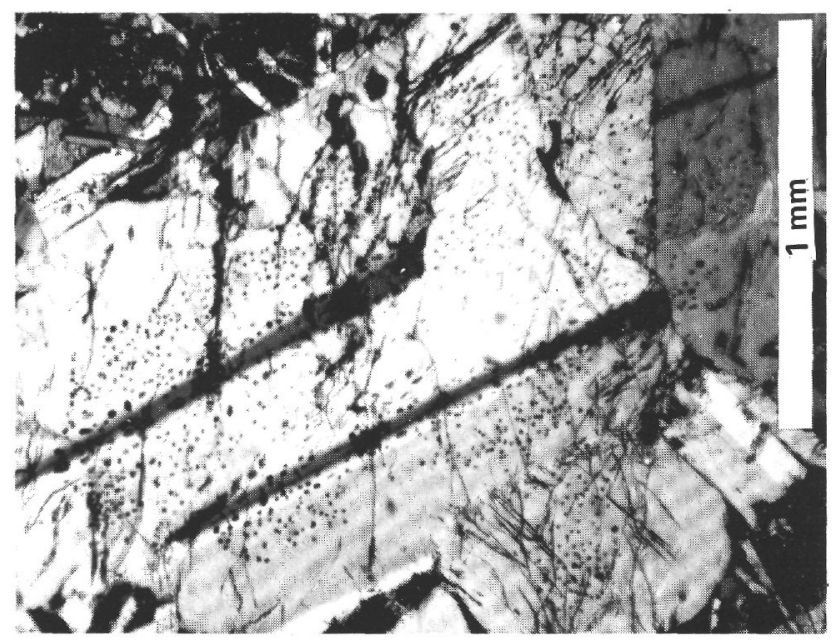

A

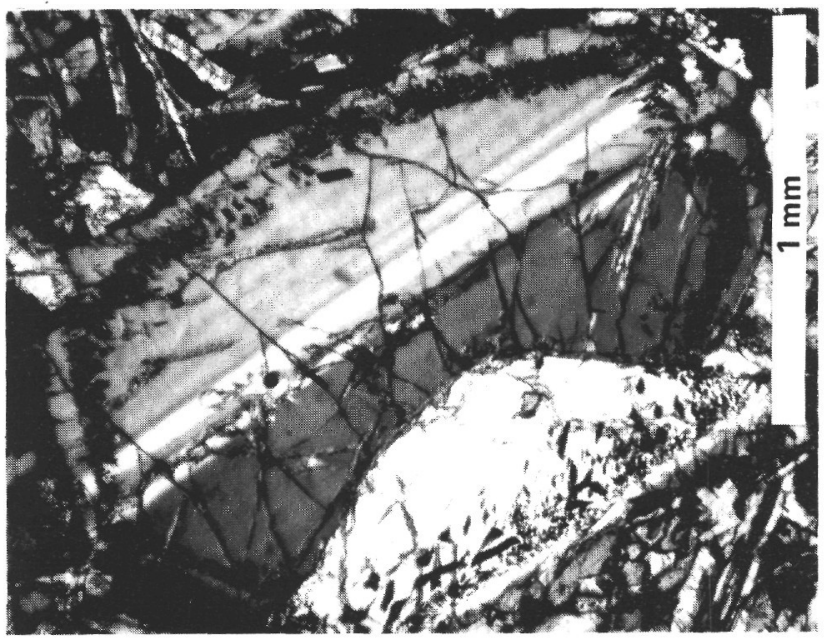

B

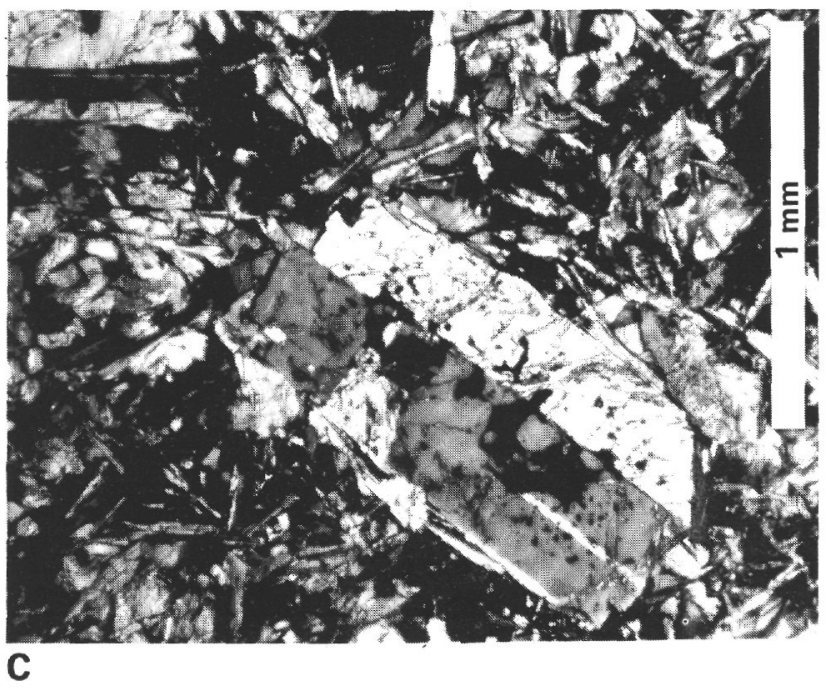

Figure 7. (A) Sample 504B-104-2, 24-27 cm. Plagioclase xenocryst texture in which core area contains numerous melt inclusions. Surrounding rim is inclusion free. Crossed polars. (B) Sample 504B-90-4, 49-53 cm. Plagioclase xenocryst texture in which devitrified melt inclusions occur at some intermediate position between core and rim. Core and rim are inclusion free. Crossed polars. (C) Sample 504B-141-1, $16-20 \mathrm{~cm}$. Plagioclase xenocryst texture in which the core is riddled by large vermicular cavities and is surrounded by an inclusion-free rim. Crossed polars.

\section{ALTERATION}

Basalts from Hole 504B are well suited for evaluating the effects of alteration on some major and trace elements. They are remarkably uniform in composition and exhibit a relatively wide range in degree of alteration. Samples studied are those selected away from veined and brecciated areas. These reflect the minimum alteration undergone by the lithologic unit. They are also more representative of the alteration to which the major portion of the basalts have been subjected. The alteration has been studied by three methods: petrographic analysis, XRD of whole-rock samples, and electron microscopic analysis of secondary mineral phases. Because of their small abundances, several secondary minerals generally cannot be identified from X-ray patterns. Therefore, the distribution of most minerals with depth is based on petrographic and microprobe analyses and is given in Figures 19A and B. Also given for comparative purposes is the distribution of vein minerals identified by the shipboard party using petrographic and XRD analyses. The relative degree of alteration is indicated by an alteration index and is defined according to the following criteria: $0=$ no alteration; $1=$ saponite replacement of olivine and interstitial areas; 2 = alteration of plagioclase along fractures, complete replacement of olivine and interstitial areas by clays or chlorite; $2^{+}=$replacement of FeTi oxides by sphene or patchy replacement of plagioclase by a zeolite or albite in addition to the criteria for $2 ; 3=$ conditions specified in $2^{+}$plus actinolite needles replacing clinopyroxene or interstitial areas; 4 = conditions specified in 3 plus alteration distributed as large patches not restricted to particular primary crystals; implies extensive alteration.

Although the alteration of the basalts certainly represents a continuum of changing conditions, this continuum can, in effect, be thought of in terms of a high- and a low-temperature event. The high-temperature phases 


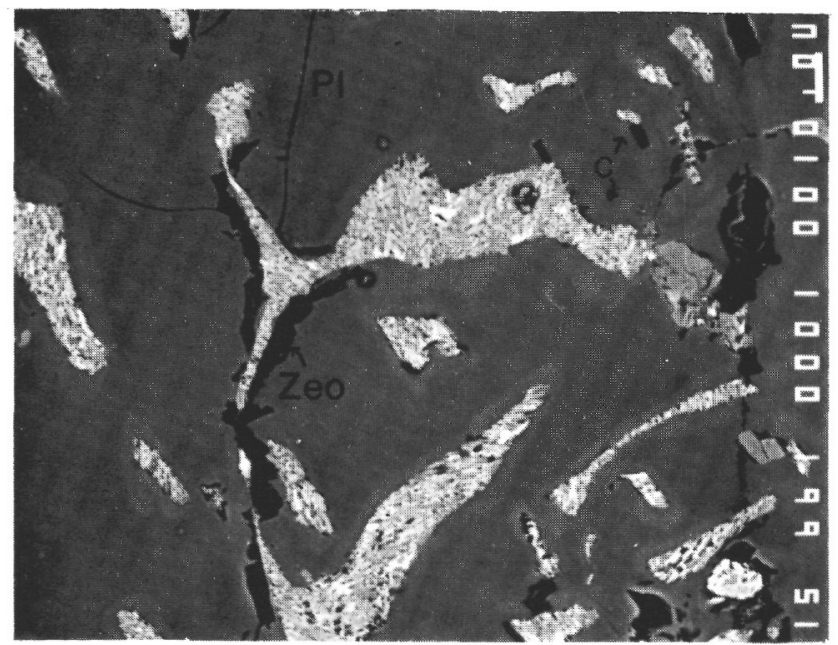

A

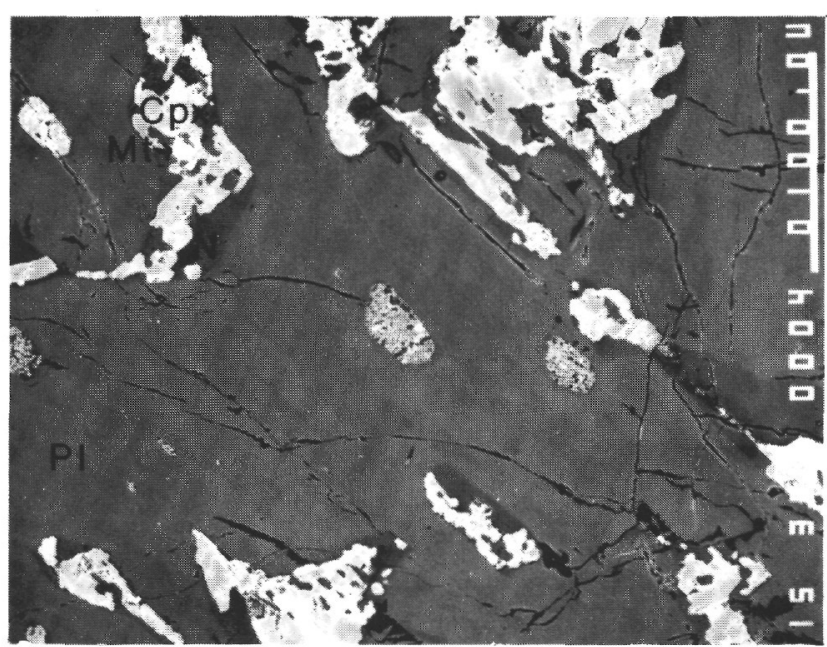

B

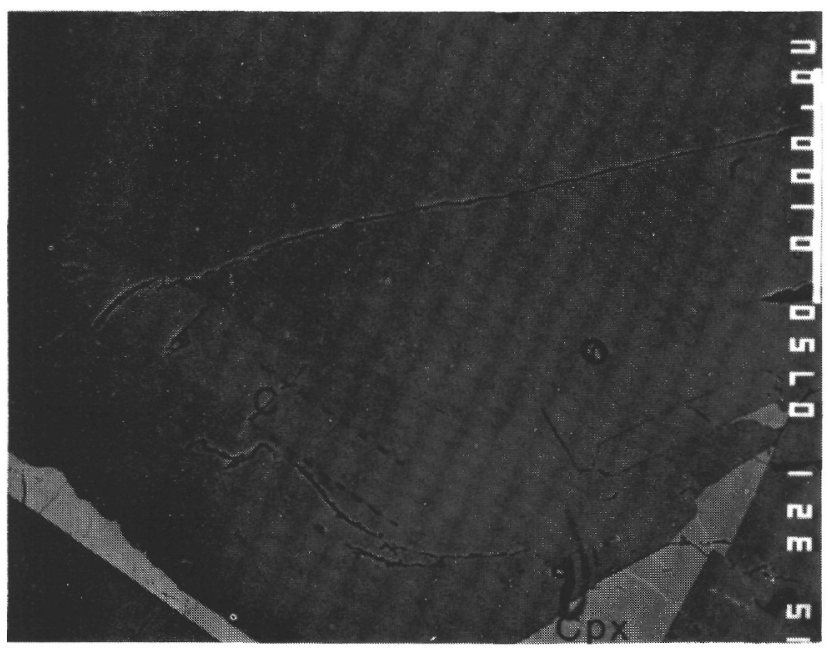

C

Figure 8. (A) Sample 504B-90-4, 49-53 cm. BEI photo of devitrified melt inclusions in xenocryst pictured in Figure 7B. Xenocryst core composition is $\mathrm{An}_{76}$; maximum phenocryst composition in $\mathrm{An}_{85}$. Notice the calcic plagioclase rims (brighter image) adjacent to the melt inclusions. The phases comprising the melt inclusions are probably clinopyroxene, plagioclase, and magnetite. $\mathrm{Pl}=$ plagioclase, $\mathrm{C}=$ calcic zone adjacent to inclusions, Zeo = zeolite. (B) Sample 504B-104-2, 24-27 cm. BEI photo of small devitrified melt inclusions plus larger cavities filled with more coarsely crystallized clinopyroxene, magnetite, and plagioclase. The xenocryst core composition is $\mathrm{An}_{88}$, while phenocryst compositions are approximately $\mathrm{An}_{78}$. Notice the more sodic plagioclase composition adjacent to the inclusions. $\mathrm{Cpx}=\mathrm{clinopyroxene,} \mathrm{Mt}$ $=$ magnetite, $\mathrm{N}=$ more sodic plagioclase zone. (C) Sample 504B-104-2, 24-27 cm. BEI photo of a phenocryst which coexists with the xenocryst pictured in Figure 8B. Phenocryst compositions $\left(\mathrm{An}_{78}\right)$ is uniform from the core outward except for a thin calcic zone $\left(A n_{86}\right)$ near the rim. A portion of the calcic zone, $\mathrm{c}$, is outlined by dashed lines.

observed include chlorite, talc, albite, actinolite, sphene, quartz, and pyrite. The low-temperature phases present are smectite (saponite), epistilbite or laumontite, and minor calcite. The zeolite minerals in the whole-rock samples occur in abundances too small to detect by XRD techniques. Their identification is based primarily on the $\mathrm{Si} / \mathrm{AlIV}$ ratio as determined from microprobe analysis and on $\mathrm{H}_{2} \mathrm{O}$ content as inferred from deficiency in analysis totals. Laumontite may actually be stable in a temperature range that overlaps both high- and lowtemperature mineral assemblages. The relative timing of alteration events cannot be conclusively established on the basis of textural relationships in whole-rock samples. However, cross-cutting relationships between lowand high-temperature mineral assemblages in veins indicate that alteration progressed in a series of stages in which the low-temperature secondary phases occur as a retrogressive overprint of the higher temperature assemblage (Alt et al., this volume). Secondary minerals observed in veins, but not present in whole-rock samples include epidote, prehnite, scolecite, and anhydrite.

In general, alteration is restricted to partial replacement of specific primary phases; olivine and interstitial material (glass \pm feldspar) are the only phases consistently and entirely replaced. The secondary minerals will be discussed in terms of the primary phases that they replace and their position in the sequence of alteration for that phase.

\section{Petrography and Mineral Chemistry}

Olivine has been altered to one or more phyllosilicates throughout the lower portion of Hole 504B. Pseudo- 

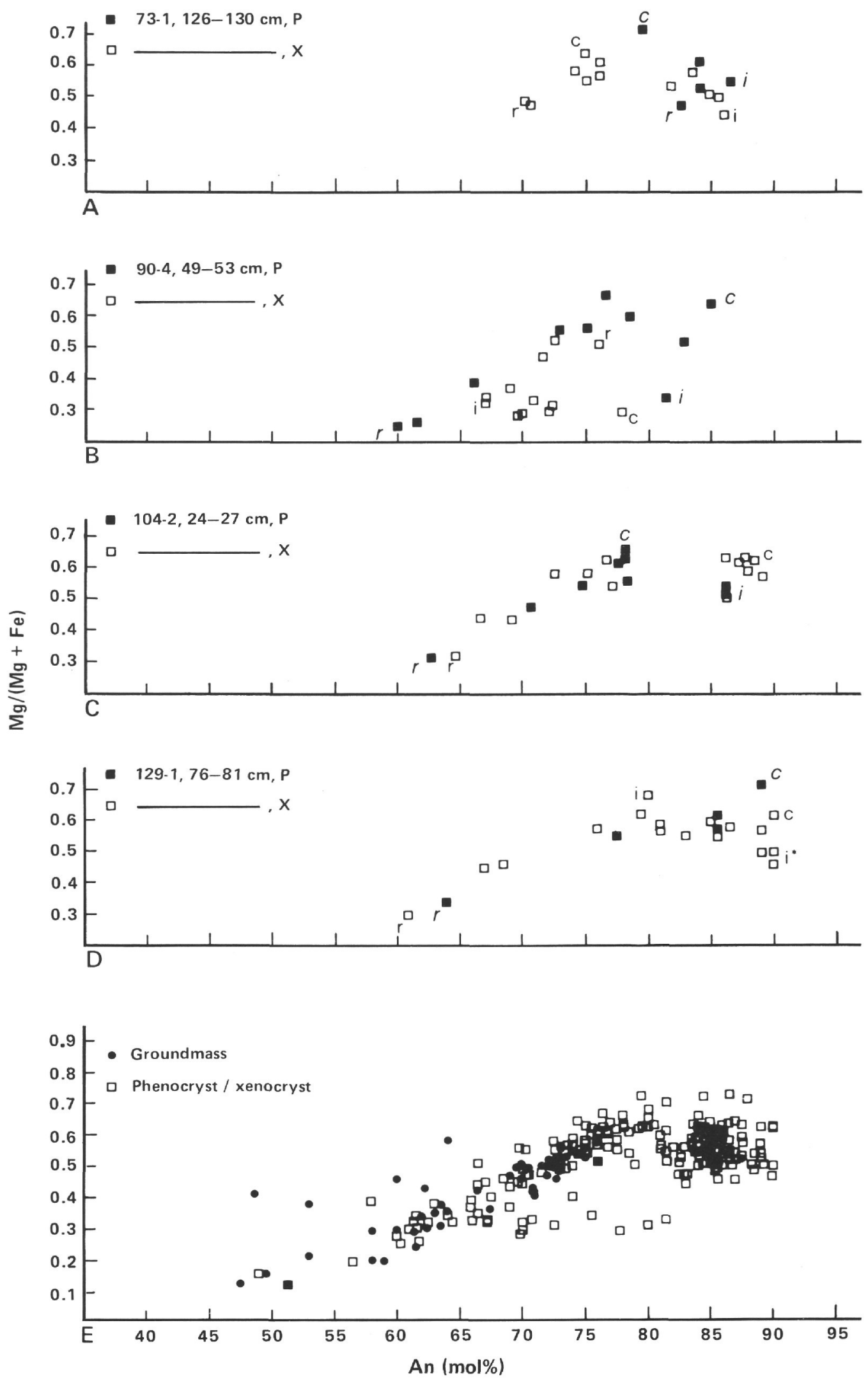

Figure 9. Plot of mol\% An vs. $\mathrm{Mg} /(\mathrm{Mg}+\mathrm{Fe})$ in plagioclase. (A-D) Representative trends for specific coexisting phenocrysts and xenocrysts. $\mathrm{P}=$ phenocryst; $\mathrm{X}=$ xenocryst. Core (c), $\operatorname{rim}(\mathrm{r})$, and an intermediate position (i) in the traverse are marked to delineate compositional trends. Xenocryst analysis locations (c, r, and i) are given in italic letters, phenocrysts in roman (block) letters. Note the wide range of zoning trends in both phenocrysts and xenocrysts from Hole 504B. (E) Includes data from all analyzed phenocrysts, xenocrysts, and groundmass crystals. 


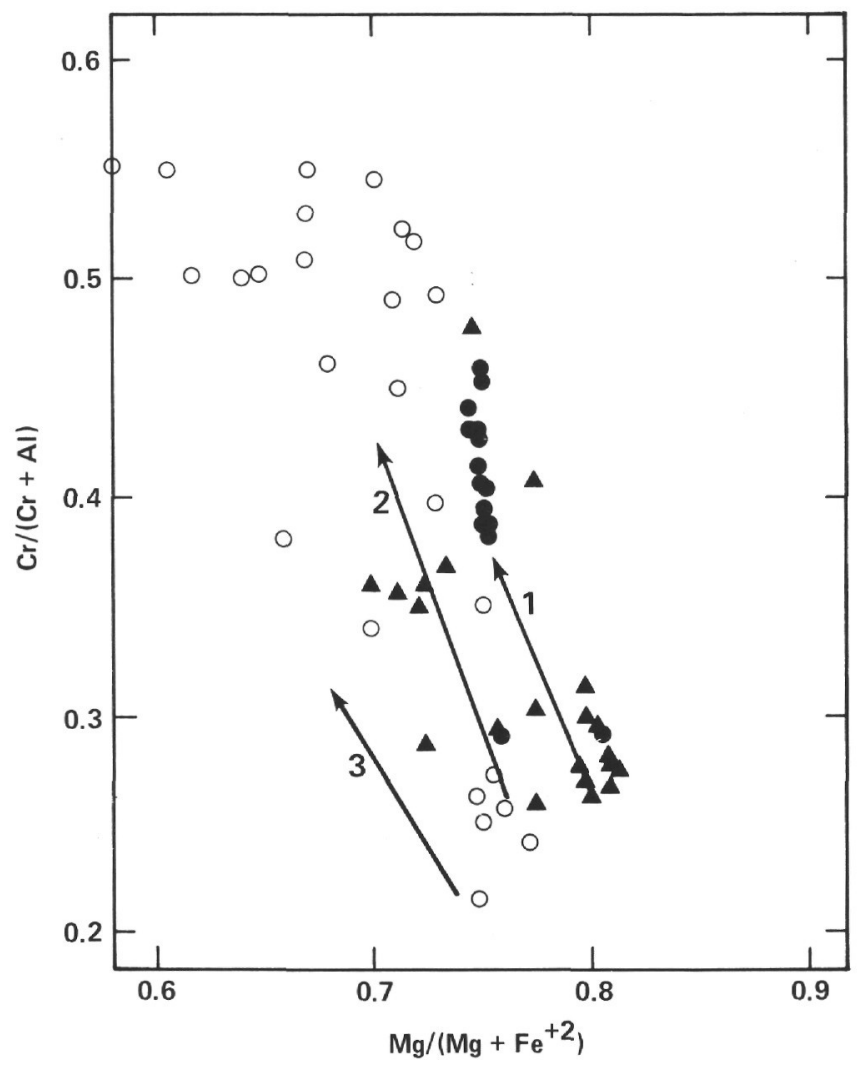

Figure 10. Microprobe analyses of spinels. Hole 504B spinels enclosed in plagioclase or olivine in fine- to medium-grained basalts. $\boldsymbol{\Delta}$, Hole 504B spinels occurring in dike or pillow chilled margins. $\mathrm{O}$, spinels from Mid-Atlantic Ridge basalts (Dungan and Rhodes, 1978; Sigurdsson and Schilling, 1976). Arrows designate average zoning trends in spinels from the Siqueiros Fracture Zone (near the East Pacific Rise) that crystallized from liquids with $\mathrm{Mg} /(\mathrm{Mg}+$ $\mathrm{Fe}^{+2}$ ) ranging from 0.71 (1) to 0.65 (3) (Natland et al., 1983).

morphs consist of (1) smectite (saponite); (2) smectite + talc + magnetite; (3) talc + magnetite; (4) chlorite; (5) chlorite + quartz; and (6) chlorite + actinolite (Figs. 20 A-D). Replacement of olivine by chlorite + quartz is observed only in Samples 504B-81-1, 64-68 cm and 504B-84-1, 77-79 $\mathrm{cm}$. This interval corresponds to a zone of extensive alteration and sulfide mineralization in Hole 504B and has been described as a stockwork (Anderson et al., 1982). Chlorite + actinolite pseudomorphs occur in this zone (Sample 504B-90-4, 49-53 cm) and near the bottom of the hole where formation of amphibole is again common (Sample 504B-137-1, 11-16 cm). Above and below this zone, olivine is generally altered to smectite \pm talc \pm magnetite. The smectite, identified as saponite, ranges from pale brown to dark golden brown in color. In smectite + talc pseudomorphs the phyllosilicates occur in an alternating, concentric arrangement.

A significant degree of the compositional variation in the secondary mineral phases observed at low grades is believed to be due to the existence of metastable mineral phases that have preserved structural or chemical elements of the minerals they replaced. Olivine in many portions of Hole 504B was initially altered to talc + magnetite. Most talc, however, was later replaced at lower temperatures by smectite by the addition of $\mathrm{H}_{2} \mathrm{O}, \mathrm{Al}$, and minor $\mathrm{Ca}$. Those smectites formed after talc are lower in $\mathrm{Al}, \mathrm{Ca}$, and $\mathrm{Fe}$ and higher in $\mathrm{Mg}$ and $\mathrm{Si}$ than interstitial smectite or smectite produced directly from olivine (Fig. 21 and Table 9). Variations in compatible trace elements substantiate this alteration sequence. The replacement of olivine by smectite preserves the concentration of Ni originally present in the olivine. Replacement of olivine by talc + magnetite, however, partitions the Ni into both magnetite and talc, effectively reducing its concentration in the phyllosilicate phase. Later replacement of talc by smectite results in lower Ni concentrations than in smectite formed directly from olivine (Table 9). The composition of the smectite after talc is also related to the availability of aluminum. The liberation of aluminum during the replacement of calcic plagioclase by albite is the principal source of this element. In samples where plagioclase is unaltered, the smectites after talc are typically low in aluminum (Fig. 21). Overprinting of the chlorite alteration by smectite is not observed due to the stability of chlorite to relatively low temperatures.

Plagioclase is partially altered to chlorite or smectite \pm albite \pm a zeolite mineral. Both zeolites, epistilbite and laumontite, replace plagioclase, but epistilbite is more common. Chlorite and smectite are confined to an occurrence in fractures that cross cut the plagioclase (Figs. 20E, F). Interstitial areas and fractures in plagioclase are always replaced by the same phyllosilicate mineral. Zeolite and albite replacement occurs in irregularly shaped patches. At depths greater than $1090 \mathrm{~m}$ BSF plagioclase is largely unaltered. Only chlorite along fractures and very minor replacement by albite occur. In the interval between 920 and $1190 \mathrm{~m}$ BSF where alteration is most pervasive, both albite and epistilbite as well as chlorite are observed. Alteration of plagioclase is most extensive in this zone, but complete replacement does not occur. In fact, groundmass crystals are commonly unaffected throughout the hole. Replacement of plagioclase by epistilbite between 920 and 1190 m BSF is the only low-temperature overprint recorded in the basalts for that interval. At depths shallower than $920 \mathrm{~m}$, replacement of plagioclase is principally by epistilbite in patches and smectite rather than chlorite along fractures.

Clinopyroxene is generally unaltered throughout the lower portion of Hole 504B. In extensively altered samples between 965 and 1030 m BSF, overgrowths of actinolite or minor replacement by chlorite develop adjacent to altered interstitial areas. The majority of the clinopyroxene, however, is unaffected. Deeper than $1190 \mathrm{~m} \mathrm{BSF}$ clinopyroxene develops a slight dusty brown appearance, particularly in the Fe-rich rims of zoned crystals. This appearance is due to a very fine-grained alteration phase that is unidentified.

FeTi-oxides are altered to sphene throughout much of Hole 504B (Leg 83). Between 965 and $1160 \mathrm{~m}$ BSF they are completely altered and at depths greater than $1270 \mathrm{~m}$ BSF they are partially altered. Between 1160 and $1270 \mathrm{~m}$, FeTi-oxides are essentially pristine. The sphene is characterized by a very fine, granular morphology, typical of sphene after magnetite (Figs. 5B, C) (Hagger- 

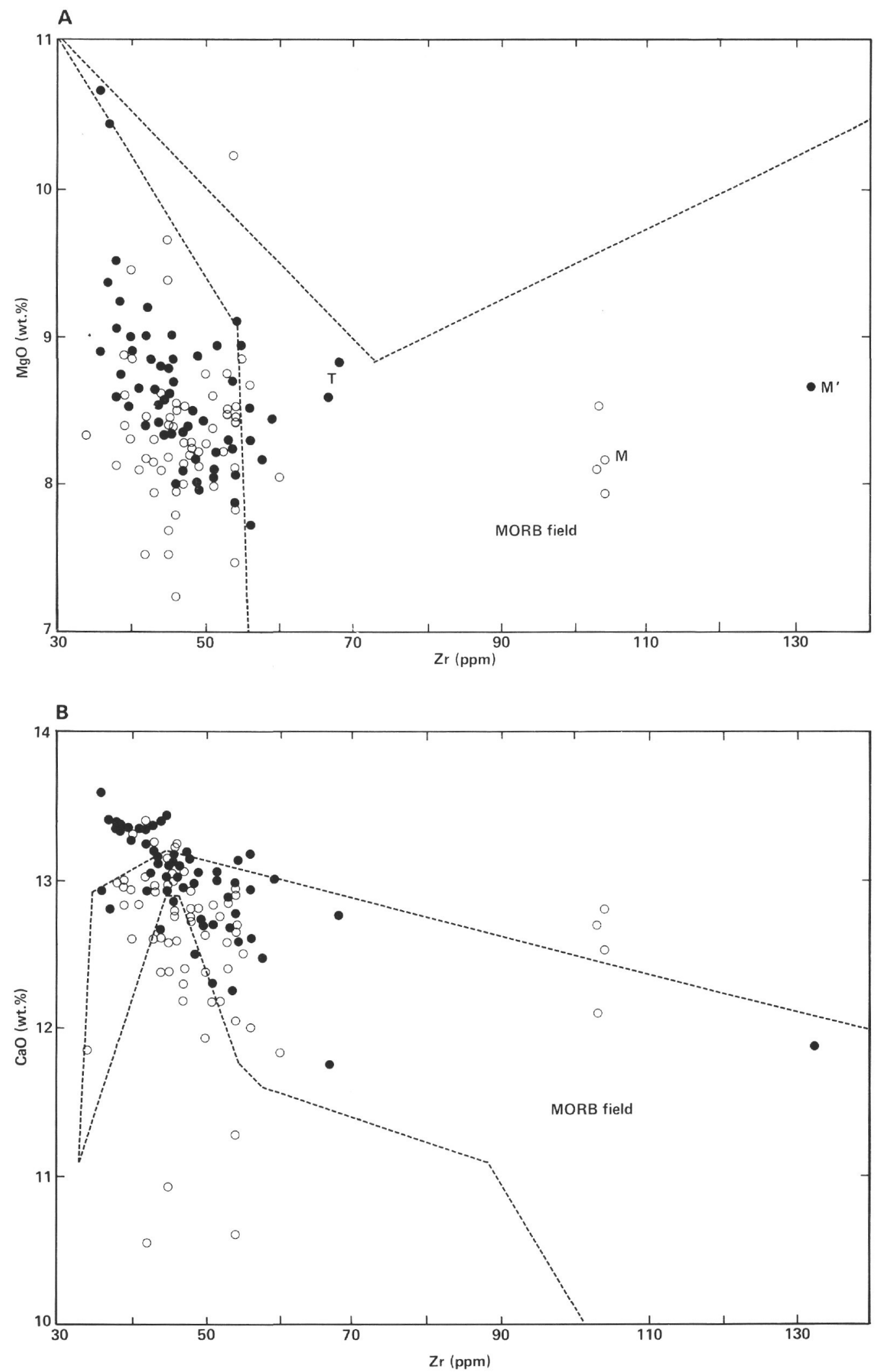

Figure 11. (A-D) $\mathrm{MgO}, \mathrm{CaO}, \mathrm{Al}_{2} \mathrm{O}_{3}$, and $\mathrm{FeO} * / \mathrm{MgO}$ vs. $\mathrm{Zr}$. $\bigcirc$, data from Legs 69 and 70 (Autio and Rhodes, 1983). , data from Leg 83 (this chapter). The Hole 504B CRRZ basalts are depleted in Zr relative to most MORB. $\mathrm{CaO}$ and $\mathrm{Al}_{2} \mathrm{O}_{3}$ abundances are higher than normal; however, $\mathrm{MgO}$ and $\mathrm{FeO} * / \mathrm{MgO}$ values are typical of other MORB of a similar state of magmatic evolution (generally olivine + plagioclase \pm clinopyroxene on the liquidus). 

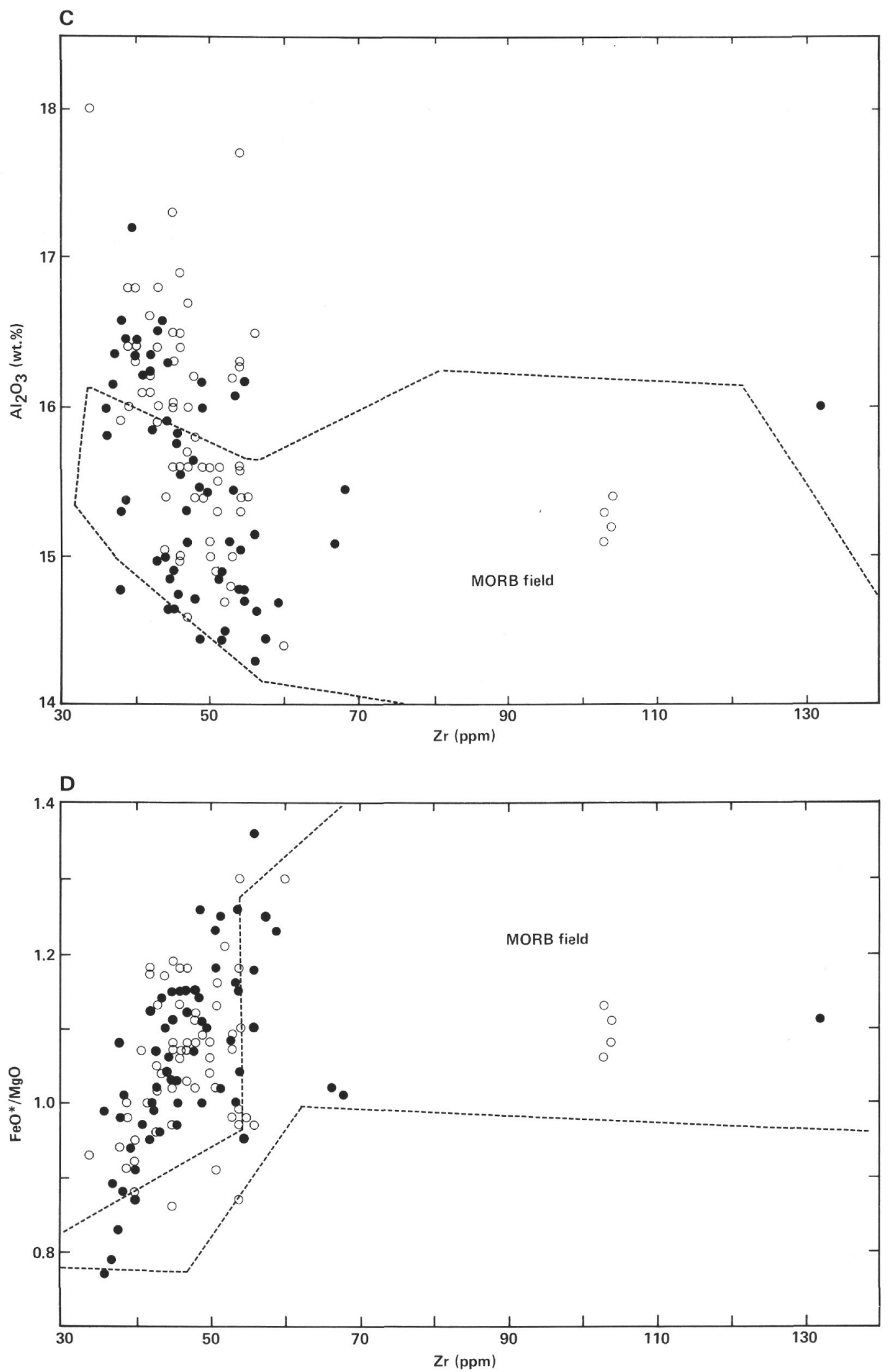

Figure 11. (Continued). 


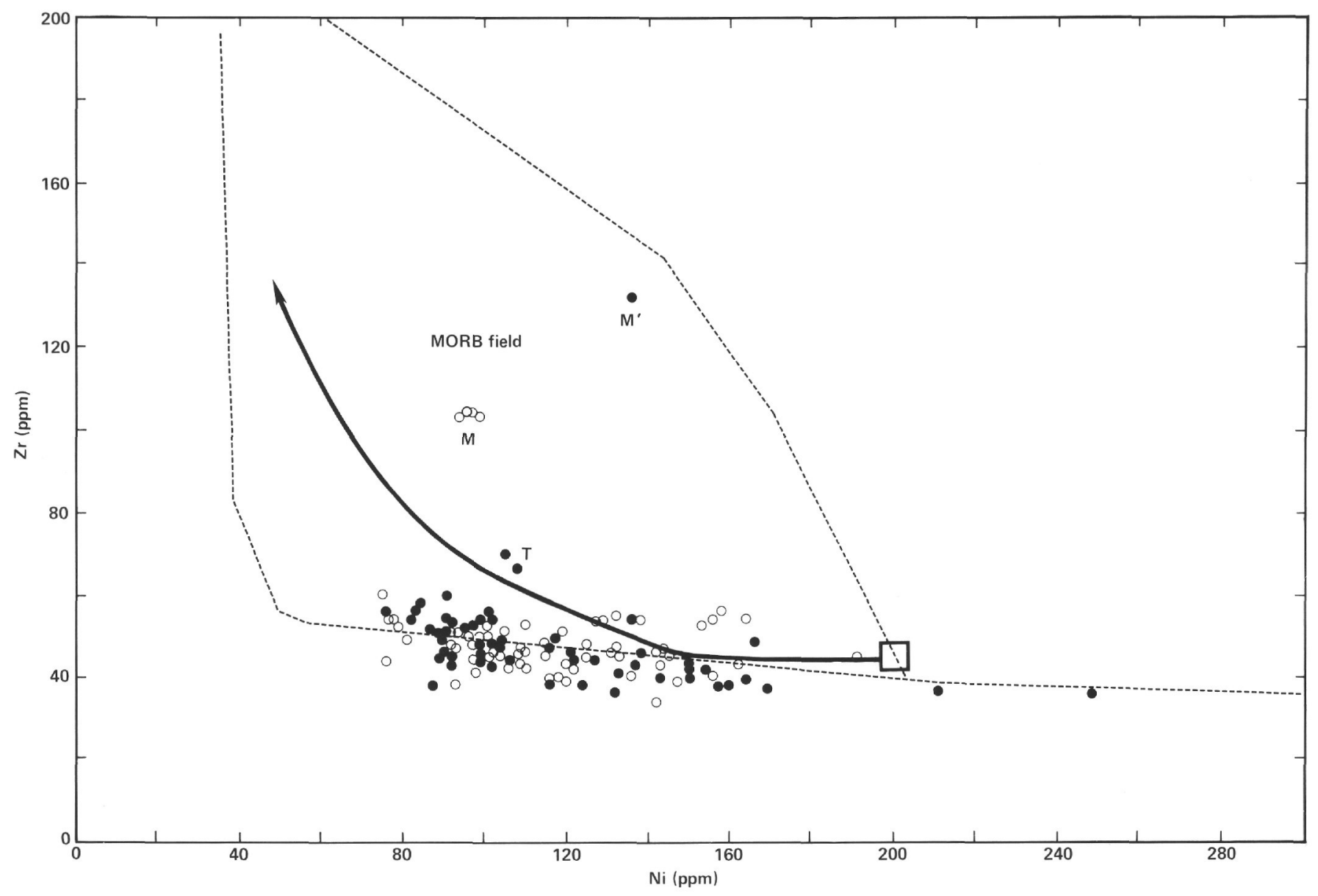

Figure 12. Zr vs. Ni. Symbols are the same as in Figure 11. A calculated fractionation trend is shown for one of the most primitive MORB collected to date (Frey et al., 1974, $\square$ ), modeled as olivine, followed by plagioclase, followed by clinopyroxene (see text). Most CRRZ basalts would follow a trend similar in configuration to that shown, but depleted in $\mathrm{Zr}$ relative to the vast majority of MORB. Groups $\mathrm{M}^{\text {and }} \mathrm{M}^{\prime}$ are more enriched and similar to "normal" MORB.

ty, 1976b). The formation of sphene from magnetite requires the addition of calcium and silica. Consequently, sphene is most common in those samples in which plagioclase is altered to albite or chlorite or both. Titanomagnetite pseudomorphs consisting of sphene + ilmenite were identified only in Sample 504B-93-3, 27-32 cm, a high-Ti, Group $\mathbf{M}^{\prime}$ basalt. In this sample, ilmenite occurs as discrete primary crystals and as discontinuous rods parallel to the (111) octahedral faces of the titanomagnetite precursor. Due to the state of alteration, it is unknown whether these composite intergrowths of magnetite and ilmenite originated from contemporaneous crystallization as an igneous process or by "exsolution" at relatively high temperatures due to increasing oxidation during cooling (see Haggerty, 1976b). Regardless, both "primary" and "secondary" ilmenite in Hole 504B are unusual among MORB ilmenites due to their high $\mathrm{MnO}$ contents (Table 2). Ilmenite, as well as titanomagnetite, is affected by sphenetization, but the ilmenite to a much lesser extent. Rare, minute crystals of ilmenite were detected in altered interstitial areas of Samples 504B-113-1, 129-133 cm and 504B-129-1, 76-81 cm by reconnaissance microprobe analyses. It could not be positively identified petrographically.
Interstitial areas, consisting originally of glass \pm feldspar react directly to fine-grained smectite above $880 \mathrm{~m}$ BSF and to chlorite below this depth. Actinolite occurs with chlorite in the interstices below 1150 m BSF. Only rarely are unaltered interstitial areas preserved in basalts from Hole 504B (Samples 504B-90-2, 101-104 cm; 504B104-2, 24-27 cm).

\section{Alteration Effects on Whole-Rock Chemistry}

Figure 22 is a plot of $\mathrm{Sr}$ vs. $\mathrm{K}_{2} \mathrm{O}$ for samples throughout the entire length of Hole 504B. The Leg 69 and 70 samples have been broken into upper (275-535 m BSF), middle (535-590 m), and lower (590-825 m) sections using the layers determined by Barrett and Friedrichsen (1982) on the basis of O and Sr isotopes. The CRRZ samples have an average $\mathrm{Sr}$ abundance $(60 \mathrm{ppm})$ that is significantly lower than normal MORB (127 ppm; Basaltic Volcanism Study Project, 1981) and a normal $\mathrm{K}_{2} \mathrm{O}$ range. $\mathrm{Sr}$ and $\mathrm{K}_{2} \mathrm{O}$ clearly vary with depth; $\mathrm{K}_{2} \mathrm{O}$ decreases dramatically and $\mathrm{Sr}$ slightly toward the bottom of the hole. $\mathrm{Rb}$ abundances measured on separate aliquots of sample from those used for $\mathrm{K}_{2} \mathrm{O}$ analysis support the compositional break occurring at the Leg 70/83 interface. This break coincides with the beginning of the 


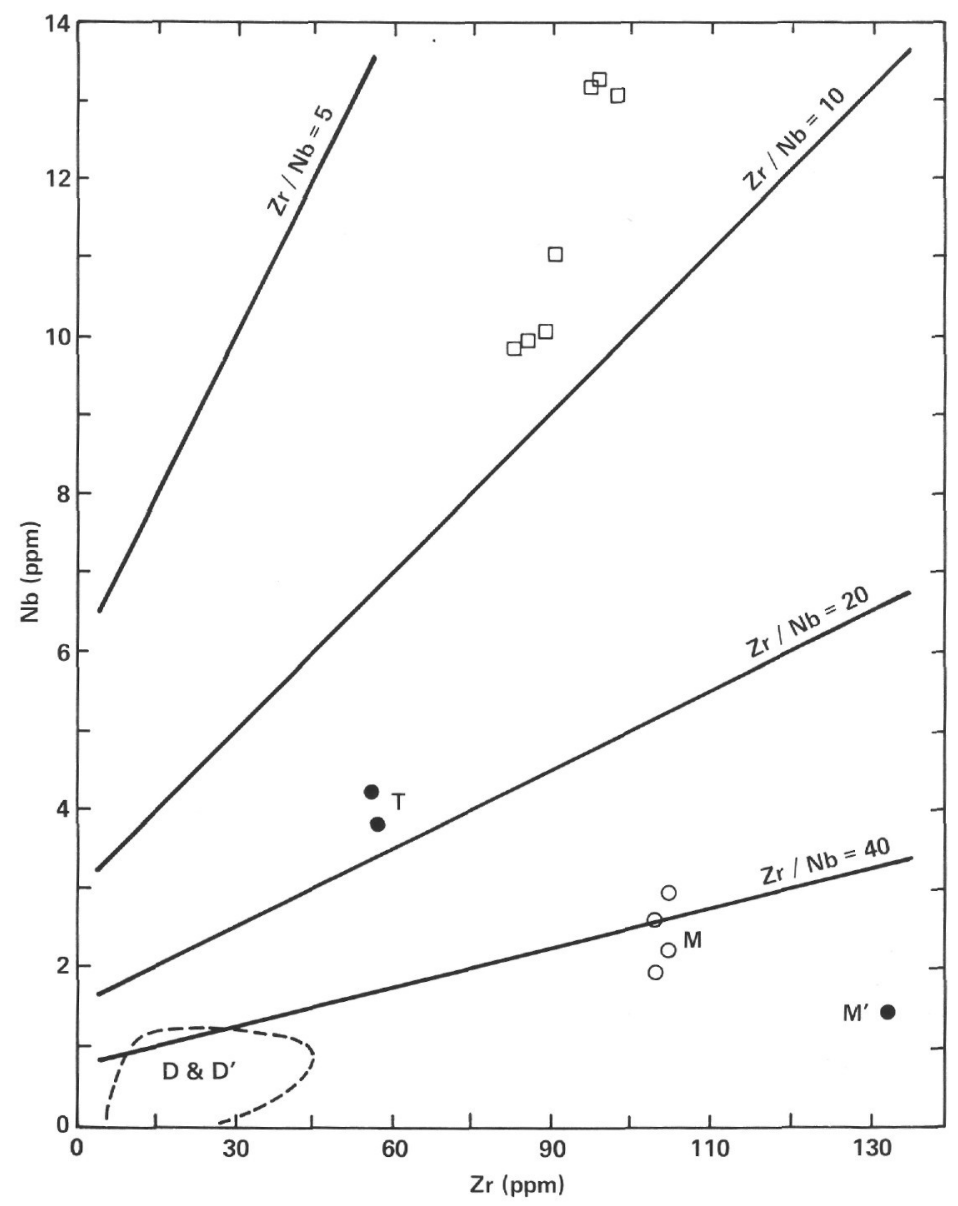

Figure 13. Nb vs. Zr. Almost all samples from Hole 504B (Legs 69, 70, and 83) plot within the area labeled D and D' (Autio and Rhodes, 1983; this chapter). Groups $\mathrm{M}(\mathrm{O})$ and $\mathrm{M}^{\prime}(\mathrm{O})$ have $\mathrm{Zr} / \mathrm{Nb} \geq 40$, similar to Groups $\mathrm{D}$ and $\mathrm{D}^{\prime}$. Group T ( ) and the high Nb group from Leg $69(\square)$ (Etoubleau et al., 1983; Marsh et al., 1983) have ratios of 17 and $\leq 10$, respectively, indicating an enriched source for these two groups compared to the other Hole 504B groups and Type I MORB (Bryan et al., 1976).

sheeted dike complex. The variation of $\mathrm{Sr}$ and $\mathrm{K}_{2} \mathrm{O}$ with depth is similar to that of the $\mathrm{O}$ and $\mathrm{Sr}$ isotopes (Barrett and Friedrichsen, 1982) that reach maximum values in the uppermost $260 \mathrm{~m}$ of basement and drop off dramatically below $825 \mathrm{~m} \mathrm{BSF}$. These changes are attributed to varying degrees of seawater alteration. Basalts below $825 \mathrm{~m}$ BSF exhibit essentially "fresh" values of $\delta^{18} \mathrm{O}$ (5.8-6.2) and ${ }^{87} \mathrm{Sr} /{ }^{86} \mathrm{Sr}(0.70266)$ (Barrett and Friedrichsen, 1982) and correspondingly lower $\mathrm{Sr}$ and $\mathrm{K}_{2} \mathrm{O}$ abundances (Fig. 22). The limited range of $\mathrm{K}_{2} \mathrm{O}$ values for Leg 83 suggests that alteration in Layer $2 \mathrm{C}$ occurred at a lower water/rock ratio than the more permeable Layer $2 \mathrm{~A}$.

A general increase in metamorphic grade from the top to the bottom of Hole 504B (Legs 69, 70, and 83) is expressed by the change in secondary mineral assemblages from smectite to chlorite dominated. However, neither composition nor degree or alteration appear to vary systematically with depth in Hole 504B for the interval recovered during Leg 83 (Fig. 19A). Degree of alteration can, however, be correlated with differences in texture and mode of emplacement. The pillow lavas and flows in the upper portion of Hole 504B (Leg 83) are typically highly fractured. They also possess fine-grained textures composed largely of glass and quench crystal morphologies. The number of intrusive units increases with depth, and basalts below $1055 \mathrm{~m} \mathrm{BSF}$ are primarily sheeted dikes and massive units. These are coarser grained and far less fractured than the overlying pillow lavas. Thus, permeability to circulating hydrothermal fluids is higher, and, consequently, the degree of alteration is greatest in the upper portion of Hole 504B. The coarser grained basalts from the lower portion of the hole are on the average less altered.

Inspection of Figure 19A also demonstrates that variations in whole-rock major and trace element compositions are not correlative with the degree of alteration. Alteration of the bulk rock proceeds primarily by local movement of ions. As a general example, the alteration of calcic plagioclase to albite releases $\mathrm{Ca}$ and $\mathrm{Al}$ that potentially could be removed from the system. However, $\mathrm{Ca}$ is taken up in the formation of sphene from magnetite, and $\mathrm{Al}$ is consumed by conversion of talc to smectite. Rigorous modeling of such reactions in terms of 


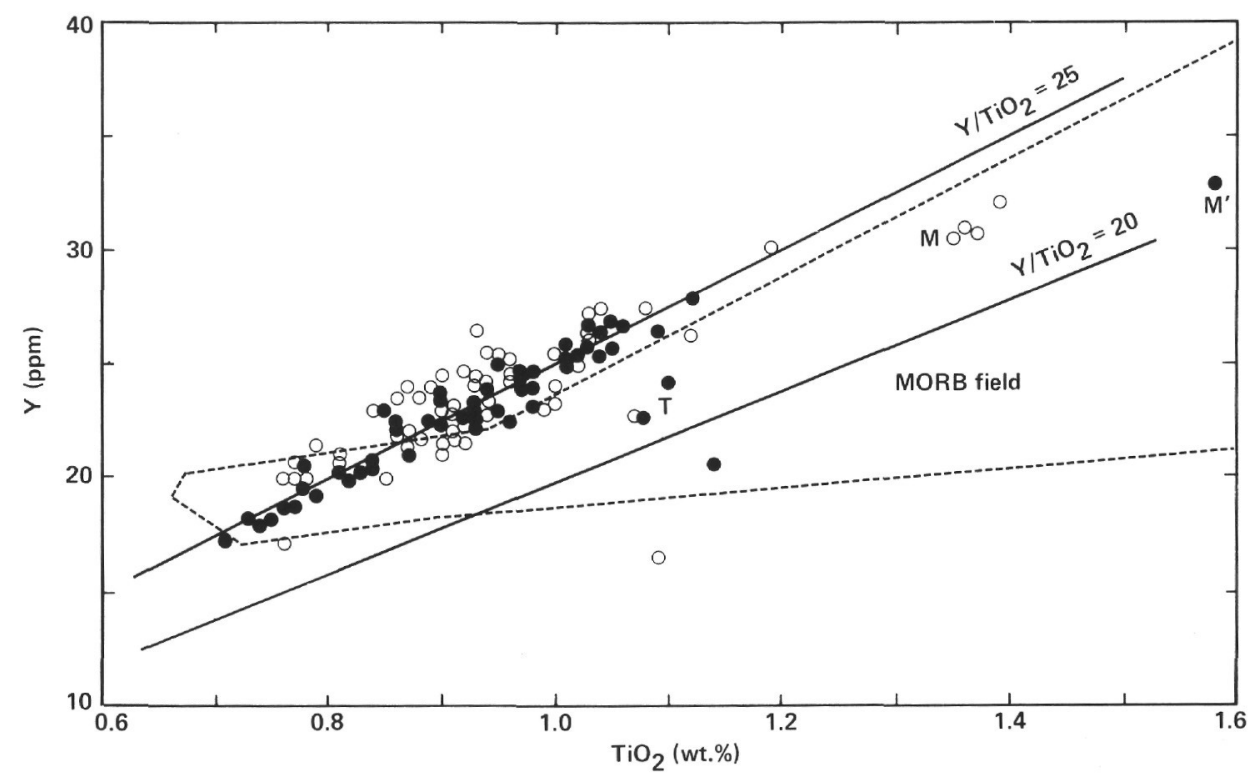

Figure 14. Y vs. $\mathrm{TiO}_{2}$. Symbols are the same as in Figure 11. CRRZ Group D and $\mathrm{D}^{\prime}$ samples are depleted in $\mathrm{TiO}_{2}$ relative to $\mathrm{Y}$ compared to other MORB. The Group $\mathbf{M}, \mathbf{M}^{\prime}$, and $\mathrm{T}$ samples are more normal in their $\mathrm{Y} / \mathrm{TiO}_{2}$ ratios $(\sim 22)$.

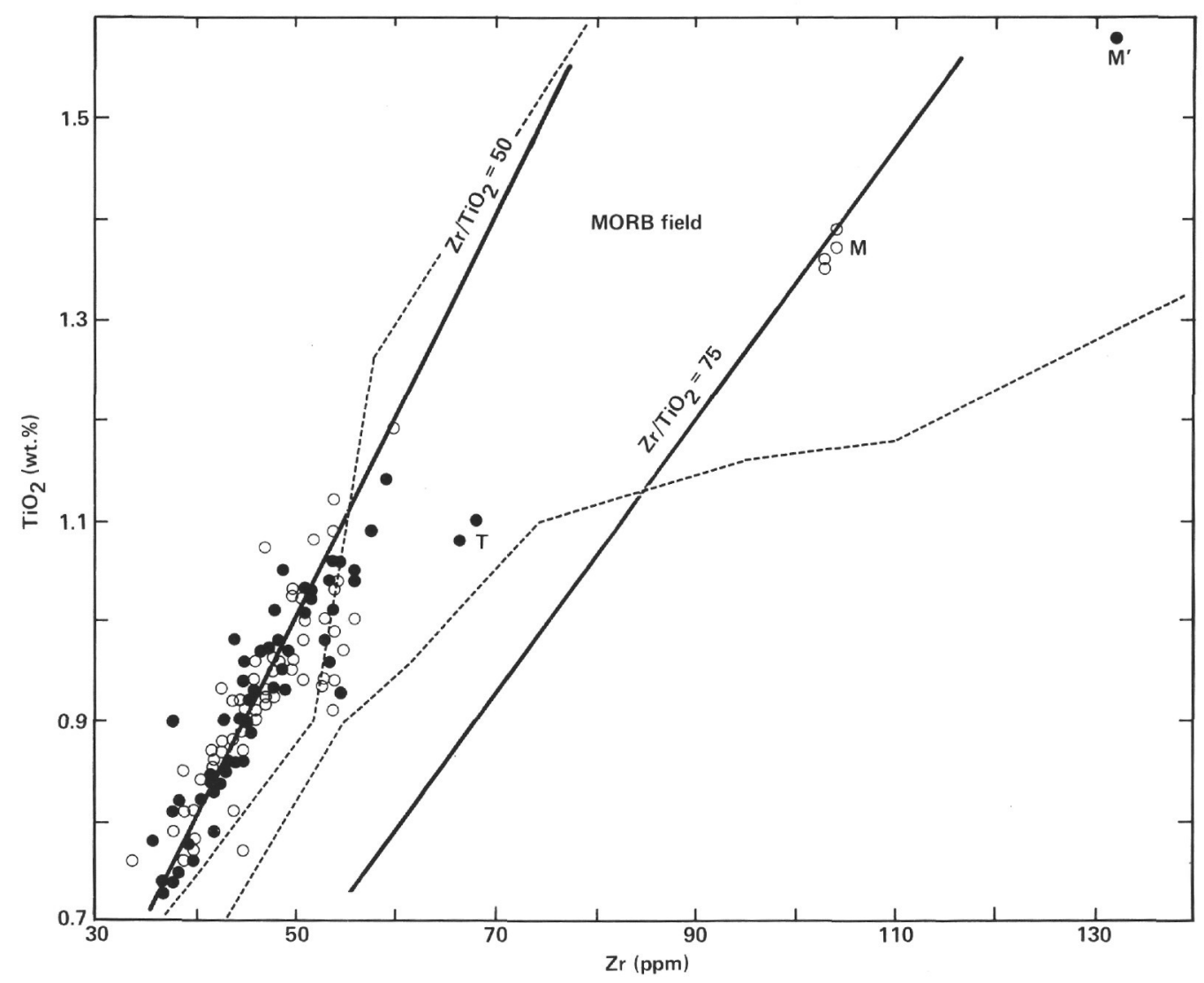

Figure 15. $\mathrm{TiO}_{2}$ vs. $\mathrm{Zr}$. Symbols are the same as in Figure 11. Most of the $\mathrm{CRRZ}$ basalts have lower $\mathrm{Zr} / \mathrm{TiO} \mathrm{O}_{2}$ ratios $(\sim 50)$ and abundances than normal MORB. Groups $\mathbf{M}$ and $\mathbf{M}^{\prime}$ are more enriched and more typical of mid-ocean ridge basalts. 


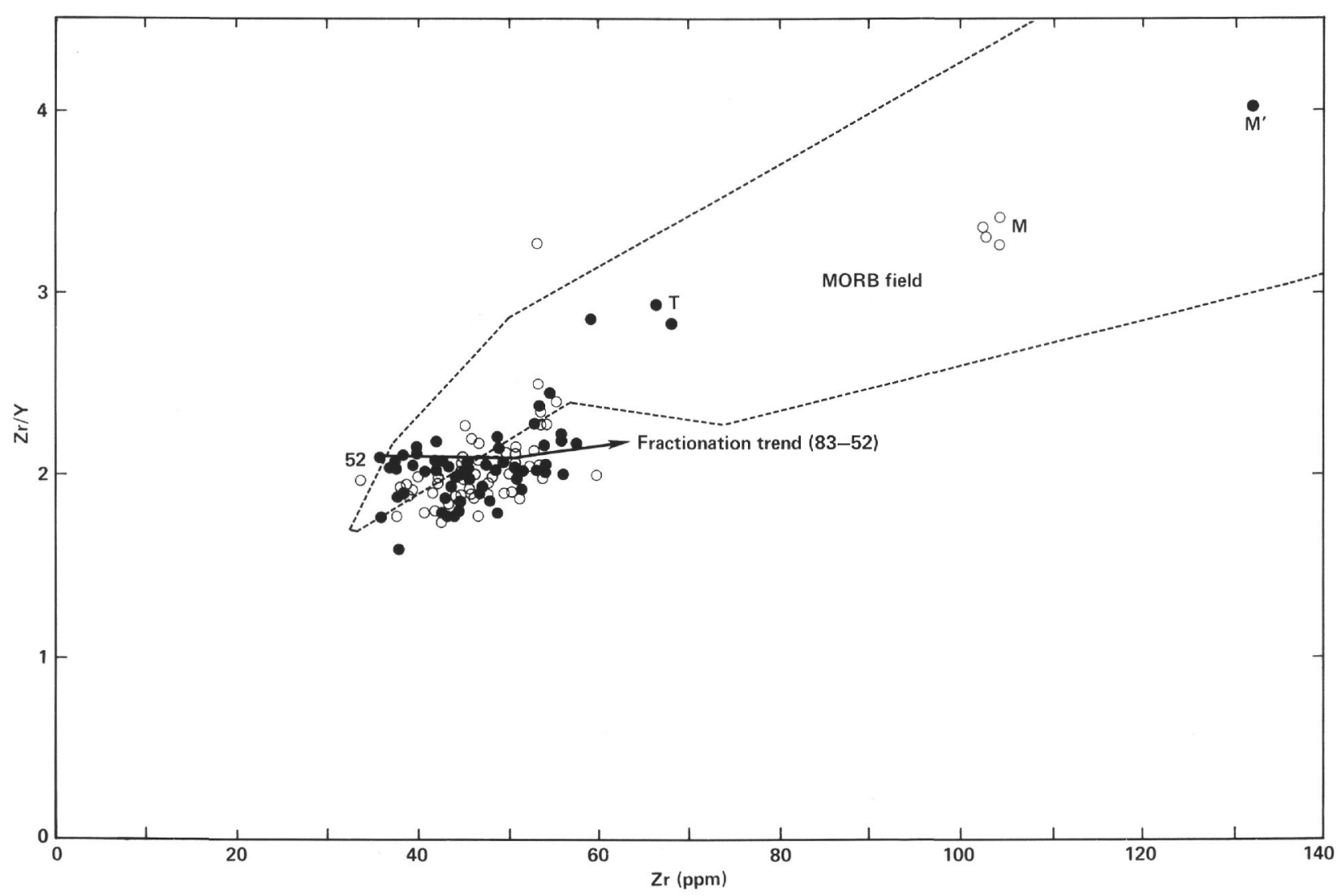

Figure 16. $\mathrm{Zr} / \mathrm{Y}$ vs. Zr. Symbols are the same as in Figure 11. Most MORB $(\sim 95 \%)$ have $\mathrm{Zr} / \mathrm{Y} \geq 2.5$. Almost all of the Hole 504B basalts (Groups D and $\mathrm{D}^{\prime}$ ) have ratios between 1.6 and 2.35. A few are higher: Groups M (3.35), $\mathrm{M}^{\prime}$ (4.00), and T (2.85). A calculated fractionation trend is shown for Sample 504B-129-1, 76-81 cm (83-52): olivine followed by plagioclase, followed by clinopyroxene. None of the groups can be related by fractionation alone, nor can the scatter within Groups D and $\mathrm{D}^{\prime}$ be accounted for solely by crystal fractionation.

mass balance has not been attempted. However, the consistent occurrence of such complementary reactions suggests that most major element characteristics of the basalts remain similar to those of the original composition. Variations in most trace elements and REE are not correlative with the degree of alteration principally because clinopyroxene, the phase containing the greatest concentration of REE and incompatible trace elements, remains essentially pristine throughout the hole.

\section{DISCUSSION}

\section{Whole-Rock Chemistry}

In an earlier section it was demonstrated that most Hole 504B basalts are compositionally distinct from typical Type I and II MORB (Bryan et al., 1976). In particular, they have very low $\mathrm{Zr}, \mathrm{Nb}, \mathrm{Hf}$, LREE, and $\mathrm{Sr}$ abundances; low $\mathrm{TiO}_{2}, \mathrm{Na}_{2} \mathrm{O}$, and $\mathrm{Y}$ concentrations; and higher than normal $\mathrm{CaO}$ abundances (Figs. 11-18). However, experimental evidence (Autio and Rhodes, 1981, 1983) suggests that the CRRZ basalts are saturated in olivine, plagioclase, and often clinopyroxene. Compatible element abundances ( $\mathrm{Ni}, \mathrm{Cr}, \mathrm{MgO}$, etc.) are similar to those observed in other moderately evolved MORB (Basaltic Volcanism Study Project, 1981). Petrographic evidence also suggests that the CRRZ basalts are multiply saturated. Thus, although these basalts are unusu- ally depleted in magmaphile elements, they are moderately evolved rather than primitive.

Basalts from Hole 504B have high $\mathrm{CaO} / \mathrm{Na}_{2} \mathrm{O}$ ratios, low $\mathrm{Zr} / \mathrm{Y}$ ratios, and low magmaphile element abundances (for their state of evolution) relative to other MORB. They also have higher than normal liquidus temperatures. These characteristics indicate that the source for the majority of CRRZ basalts is unusually depleted relative to other MORB. A liquid generated in a source that has been depleted by removal of an earlier (basaltic?) magma will be in equilibrium with a much more refractory residue. This liquid will have a higher $\mathrm{mg}$ value and perhaps even be picritic in composition. Although a few Leg 83 basalts, such as Sample 504B-129-1, $76-81 \mathrm{~cm}$, have compositional characteristics $(\mathrm{Ni}=246$ ppm, $\mathrm{Cr}=532 \mathrm{ppm}, \mathrm{mg}$ value $=0.72$ ) similar to the most primitive MORB described (Frey et al., 1974; Basaltic Volcanism Study Project, 1981), these and other depleted CRRZ basalts are inferred to be the product of extensive olivine fractionation from relatively magnesian (picritic?) melts rather than primary magmas.

As a result of their unusually high $\mathrm{CaO} / \mathrm{Na}_{2} \mathrm{O}$ ratios (a reflection of their depleted source), the CRRZ basalts are calculated to be in equilibrium with highly calcic plagioclase compositions (up to $\mathrm{An}_{88}$ ) (Drake, 1976). Microprobe analyses (Fig. 2) of groundmass plagioclase crystals confirm these high values for the most depleted 


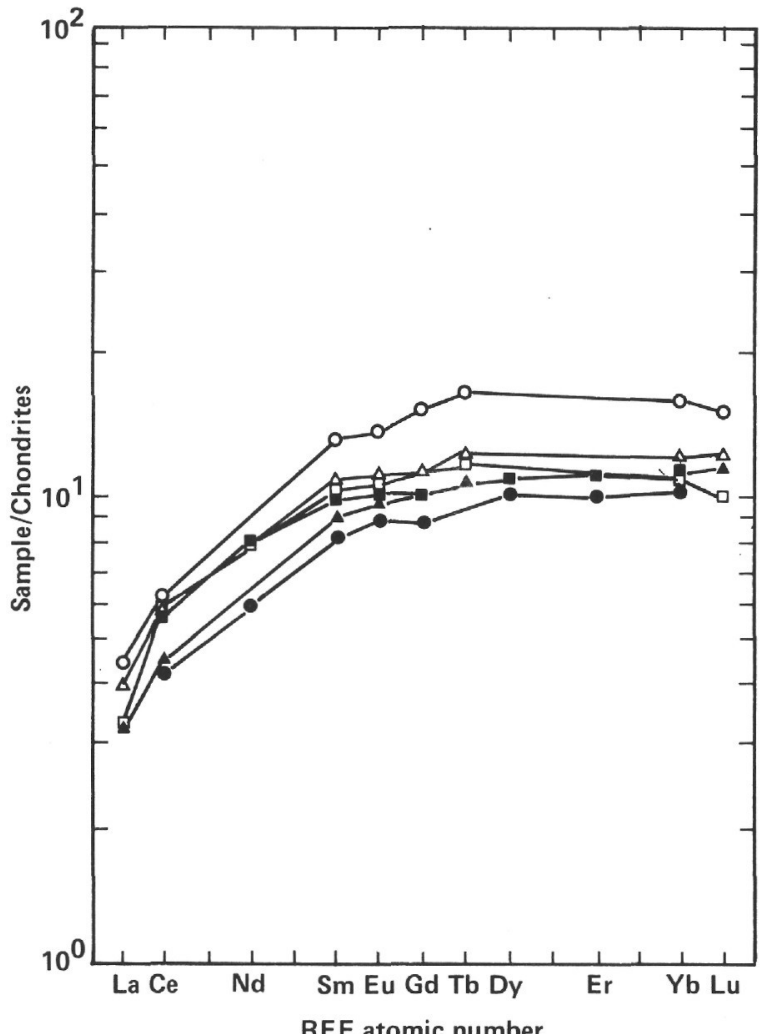

B

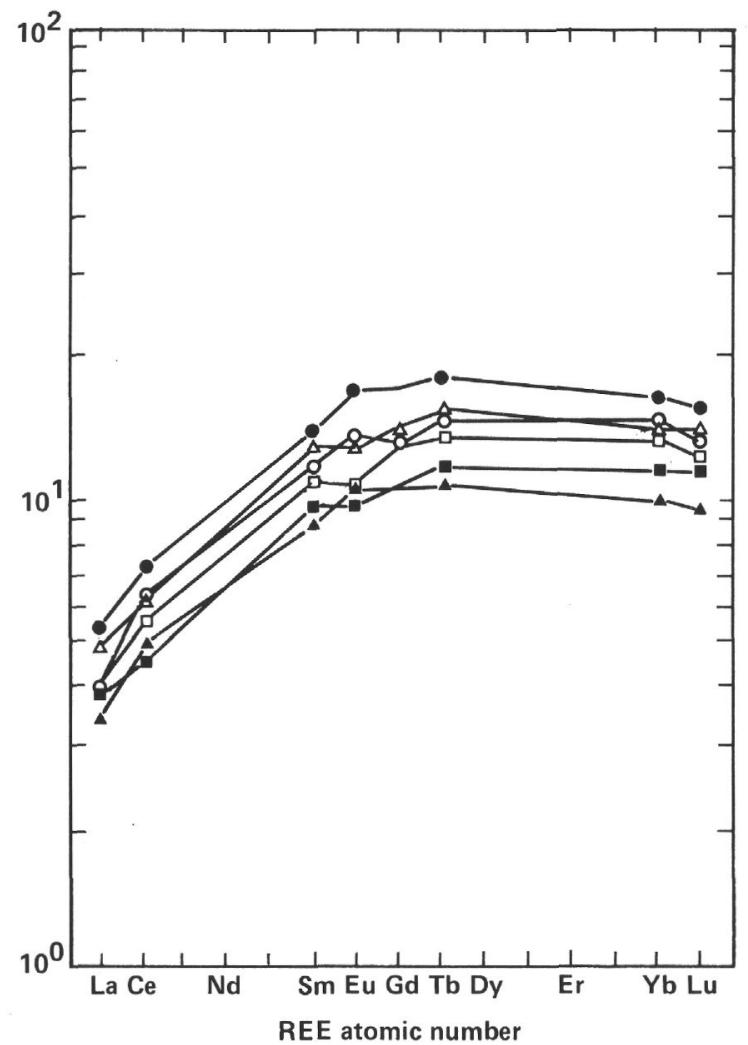

C

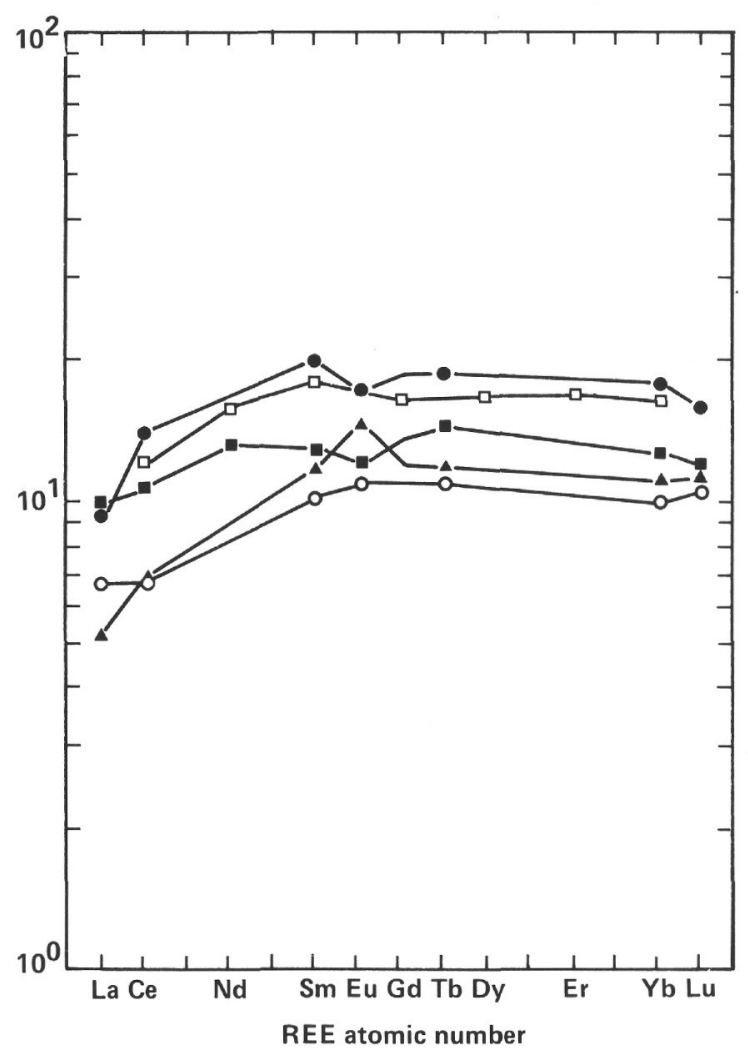

Figure 17. Chondrite normalized REE abundances for Hole 504B basalts. (A) Group D' and D basalts, Leg 70: Sample 504B-8-5, 72 cm, 0; Sample 504B-11-2, 105 cm, a. Leg 83: Sample 504B73-1, 126-130 cm $\square$; Sample 504B-77-3, 11-13 cm, O; Sample 504B-84-1, 77-79 cm, $\triangle$; Sample 504B-91-1, 127-132 cm, $\Delta$. Notice the marked depletion of LREE relative to HREE as well as the low concentrations of all REE relative to Type I MORB. (B) Group D' basalts, Leg 83: Sample 504B-104-1, 40-42 cm, O; Sample 504B-109-1, 49-52 cm, $\square$; Sample 504B-113-1, 129-133 $\mathrm{cm}, \mathbf{B}$; Sample 504B-116-1, 43-46 cm, $\triangle$; Sample 504B-129-1, 76-81 cm, $\Delta$; Sample 504B-140-1, 40-42 cm, 0 . (C) Group M basalt, Leg 70: Sample 504B-57-1, 36 cm, $\square$. Group M' basalt, Leg 83: Sample 504B-93-3, 27-32 cm, Group T basalt, Leg 83: Sample 504B-88-1, 109-113 cm, @. Group D' (enriched) basalts, Leg 83: Sample 504B-90-4, 49-53 cm, $\Delta$; Sample 504B$141-1,16-20 \mathrm{~cm}, O$. Groups $\mathrm{M}^{\prime}, \mathrm{T}$, and enriched $\mathrm{D}^{\prime}$ basalts are less depleted in LREE relative to HREE, similar to Type I MORB. 


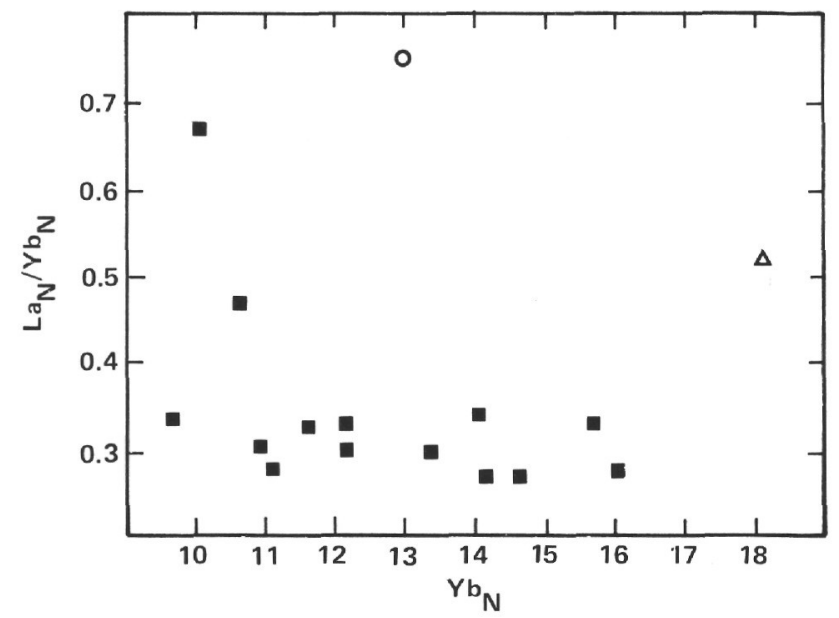

Figure 18. Plot of chondrite normalized $\mathrm{La} / \mathrm{Yb} \mathrm{vs}$. $\mathrm{Yb}$. Notice the low, uniform value of $\mathrm{La}_{\mathrm{N}} / \mathrm{Yb}_{\mathrm{N}}$ vs. $\mathrm{Yb}_{\mathrm{N}}$ for most Group $\mathrm{D}^{\prime}$ basalts ( $\square$ ) throughout the range of $\mathrm{Yb}_{\mathrm{N}} \cdot \mathrm{La}_{\mathrm{N}} / \mathrm{Yb}_{\mathrm{N}}$ values from Groups $\mathrm{M}^{\prime}(\triangle), \mathrm{T}(\mathrm{O})$, and enriched $\mathrm{D}^{\prime}$ basalts are higher, and their distribution relative to $\mathrm{Yb}_{\mathrm{N}}$ is scattered.

samples. The more calcic of these compositions are similar to the enigmatic disequilibrium plagioclase phenocrysts found in most phyric MORB samples (Bryan et al., 1976; Dungan and Rhodes, 1978; Duncan and Green, 1980). Autio and Rhodes $(1981,1983)$ have proposed that depleted magmatic compositions similar to the unusual CRRZ basalts might be a small but pervasive component of most MORB. Mixing small amounts of depleted, later magmas (and their entrained phenocrysts) with more voluminous, less-depleted, earlier melts would provide a source for the refractory plagioclases in normal MORB without greatly perturbing the magma chemistry. Only in areas where the earlier melts were not as voluminous would the depleted magmas reach the surface in a recognizable state (Autio and Rhodes, 1983).

As was noted earlier, Hole 504B penetrated predominantly flows and pillow lavas in the upper $550 \mathrm{~m}$ of the hole (Legs 69 and 70) and an increasing number of intrusive units at depth (Leg 83). Despite this lithologic change, the basalts remain similar in composition throughout the entire length of the hole (Table 6). Application of the two-tailed student's $t$-test $(99 \%$ level) to Groups D (upper Hole 504B) and $\mathrm{D}^{\prime}$ (lower) shows that there are no significant differences between the groups for most trace elements, $\mathrm{TiO}_{2}, \mathrm{Fe}_{2} \mathrm{O}_{3}{ }^{*}$, and $\mathrm{MgO}$. However, very small but statistically significant differences exist for the remaining major elements and $\mathrm{Sr}$ (Figs 11A-D and 22).

From the bottom of Hole 504B to the top, $\mathrm{CaO}(0.5$ wt. $\%$ change), $\mathrm{MnO}(0.03 \%)$, and $\mathrm{P}_{2} \mathrm{O}_{5}(0.02 \%)$ decrease slightly; whereas $\mathrm{SiO}_{2}(0.5 \%), \mathrm{Al}_{2} \mathrm{O}_{3}(0.6 \%), \mathrm{K}_{2} \mathrm{O}$ $(0.09 \%)$, and $\mathrm{Sr}(9 \mathrm{ppm})$ increase slightly. Since most trace elements and $\mathrm{TiO}_{2}$ remain essentially constant, these differences cannot be simply due to crystal fractionation. Phenocryst addition (Fig. 23), wall rock contamination and seawater alteration are possible processes for changing some elements slightly while leaving others constant. These contamination or mixing effects will be discussed later.
Groups $\mathrm{M}$ and $\mathrm{M}^{\prime}$ (upper and lower Hole 504B) are both similar to Type I MORB (Bryan et al., 1976), but they are not comagmatic. Group $\mathbf{M}^{\prime}$ has higher magmaphile element abundances $\left(\mathrm{TiO}_{2}=1.58\right.$ vs. $1.36 \%$ and $\mathrm{Zr}=132$ vs. $104 \mathrm{ppm}$ ), but also has higher compatible element abundances $(\mathrm{Ni}=134$ vs. $95 \mathrm{ppm}$ and $\mathrm{Cr}=$ 287 vs. $257 \mathrm{ppm}$ ). Therefore, Group $\mathbf{M}^{\prime}$ is not only more enriched than Group $M$, it also is more primitive.

It appears from Figure 13 ( $\mathrm{Zr}$ vs. $\mathrm{Nb}$ ) that Group T could be produced by mixing depleted (Group $\mathrm{D}^{\prime}$ ) and high $\mathrm{Nb}$ compositions. However, further calculations involving $\mathrm{TiO}_{2}, \mathrm{Y}, \mathrm{Zn}, \mathrm{Ni}, \mathrm{Cr}, \mathrm{La}, \mathrm{Sm}$, and $\mathrm{V}$ indicate major problems with this explanation. Different $\mathrm{La} / \mathrm{Sm}$ and $\mathrm{Zr} / \mathrm{Nb}$ ratios, coupled with similar $\mathrm{Zr} / \mathrm{Y}$ ratios for these two groups, preclude their being related by simple crystal fractionation or multistage melting.

Significant differences in magmaphile element abundances and ratios, coupled with similar mg values and compatible element concentrations, indicate that the different chemical groups present at the CRRZ are not comagmatic. Although Groups $\mathrm{D}$ and $\mathrm{D}^{\prime}$ seem to form a single group related by crystal fractionation, the scatter observed within these groups is too large to be accounted for by fractional crystallization, mixing of consanguineous magmas, alteration, or phenocryst addition (Autio and Rhodes, 1983).

The observed differences in magmaphile element ratios $(\mathrm{La} / \mathrm{Sm}, \mathrm{Zr} / \mathrm{Y})$ and abundances can be attributed to mantle source heterogeneity or to multistage melting processes (Schilling, 1971; Langmuir et al., 1977; Wood, 1979; Duncan and Green, 1980). However, it is difficult to change the ratios of highly incompatible elements $(\mathrm{La} / \mathrm{Ce}, \mathrm{La} / \mathrm{Nb}, \mathrm{Zr} / \mathrm{Nb}$ ) substantially by simple or multistage melting (Langmuir et al., 1977; Bougault et al., 1979). Therefore, it appears probable that the large differences in these ratios between Group $T$ basalts, the high $\mathrm{Nb}$ basalts from the top of Hole 504B, and the other basalt groups are a reflection of local mantle heterogeneity. The source for Group T must be enriched in $\mathrm{Nb}$ and $\mathrm{La}$ and to a lesser degree in $\mathrm{Zr}, \mathrm{Ti}, \mathrm{Mn}, \mathrm{P}, \mathrm{Sr}$, and $\mathrm{Ce}$ relative to the other elements. The source for the very high $\mathrm{Nb}$ basalts must be even more enriched.

The other basalt groups (D, $D^{\prime}, M$, and $\left.M^{\prime}\right)$ all have similar ratios for highly incompatible elements, but variable ratios for the less incompatible elements (e.g., $\mathrm{Zr} / \mathrm{Y}$ ). Such variation could be produced by multistage melting (Autio and Rhodes, 1983) or by mantle heterogeneity.

\section{Petrology}

Variations in mineral chemistry reflect the unusual CRRZ basalt chemistry. Compared to other MORB, feldspar compositions are more refractory (calcic), ranging up to $\mathrm{An}_{88}$ in the groundmass (Fig. 2; Bryan et al., 1976). $\mathrm{Cr}$-spinels have generally higher $\mathrm{mg}$ value for a given $\mathrm{Cr} /(\mathrm{Cr}+\mathrm{Al})$ ratio (Fig. 10; Sigurdsson and Schilling, 1976; Basaltic Volcanism Study Project, 1981; Dick and Bullen, in press; Natland et al., 1983), reflecting the high mg values of these basalts. Clinopyroxenes exhibit relatively minor enrichment in $\mathrm{Ti}$ compared to other MORB (Fig. 4B; Dungan and Rhodes, 1978; Ayuso et 


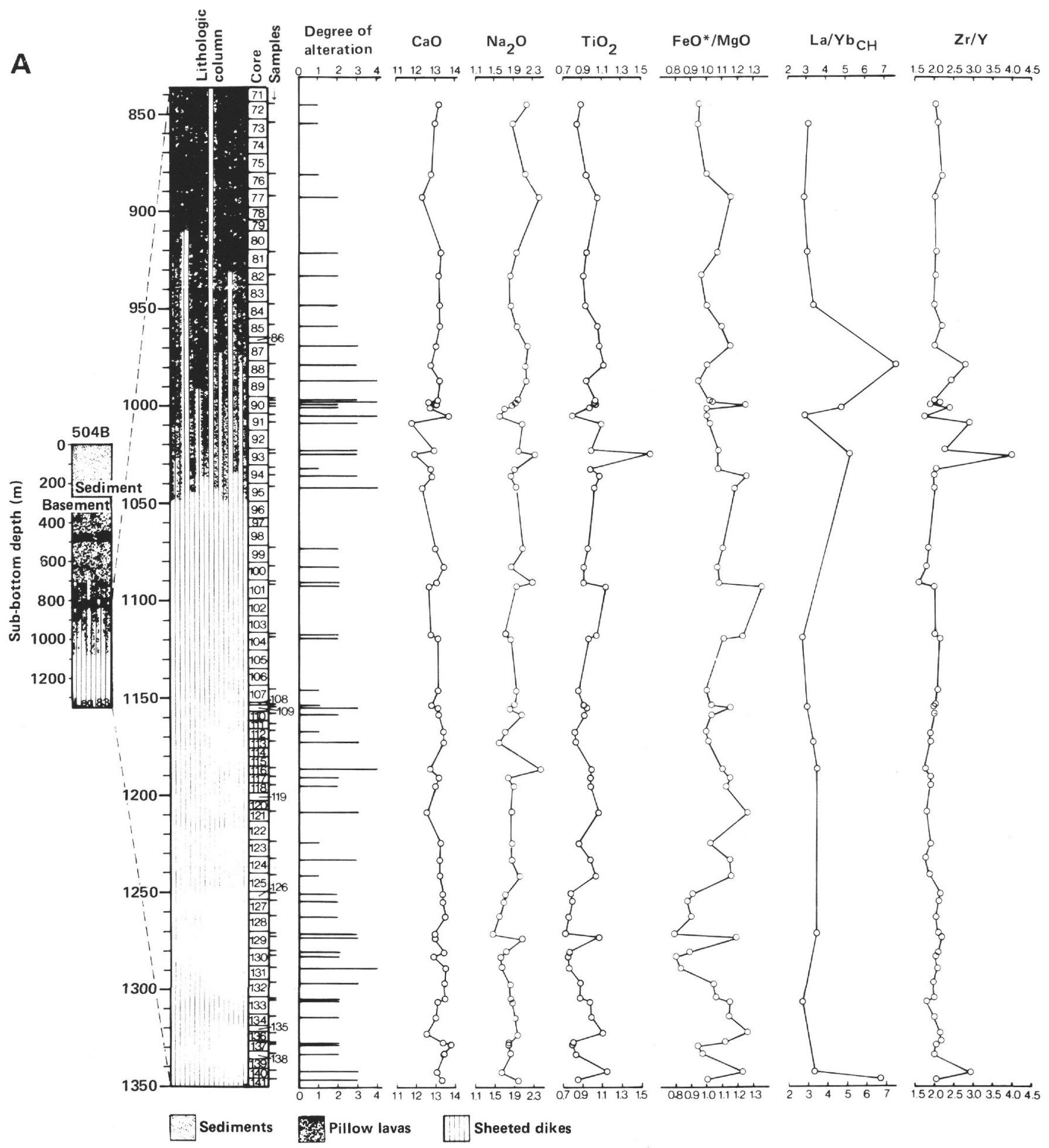

Figure 19. (A) Variation in generalized lithology; degree of alteration; $\mathrm{CaO}, \mathrm{Na}_{2} \mathrm{O}$, and $\mathrm{TiO}_{2}$ concentrations (wt. \%); and $\mathrm{FeO} * \mathrm{MgO}, \mathrm{La} / \mathrm{Yb} \mathrm{CH}_{\mathrm{H}}$, and $\mathrm{Zr} / \mathrm{Y}$ ratios with depth in Hole 504B, Leg 83. Depth is given in meters below the seafloor. The location of each sample in the hole is indicated by a tick mark adjacent to the appropriate core number. Criteria used for assigning the degree of alteration are defined in the text. (B) Secondary mineral distribution with depth. $\mathrm{O}$, secondary minerals in basalt samples, $\bigcirc$, secondary minerals localized in veins as determined by the shipboard party (Leg 83). Solid lines connect consecutive occurrences of a given mineral. Dashed lines connecting the occurrences of zeolites and albite between the depths of 886 and $1040 \mathrm{~m}$ BSF indicate the uncertainty in distinguishing these minerals petrographically in these samples. Both have been positively identified in Cores 88,90 , and $91 . \mathrm{Sm}=$ smectite, $\mathrm{Tlc}=$ talc, $\mathrm{Chl}=$ chlorite, Zeo = zeolite (either epistilbite or laumontite), $\mathrm{Ab}=$ albite, $\mathrm{Sph}=$ sphene, $\mathrm{Act}=$ actinolite, $\mathrm{Qtz}=$ quartz, $\mathrm{Cc}=$ calcite, $\mathrm{Pyr}=$ pyrite, $\mathrm{UWM}=$ unidentified white minerals (observed in veins in hand specimen only; probably includes laumontite, quartz, and calcite). 


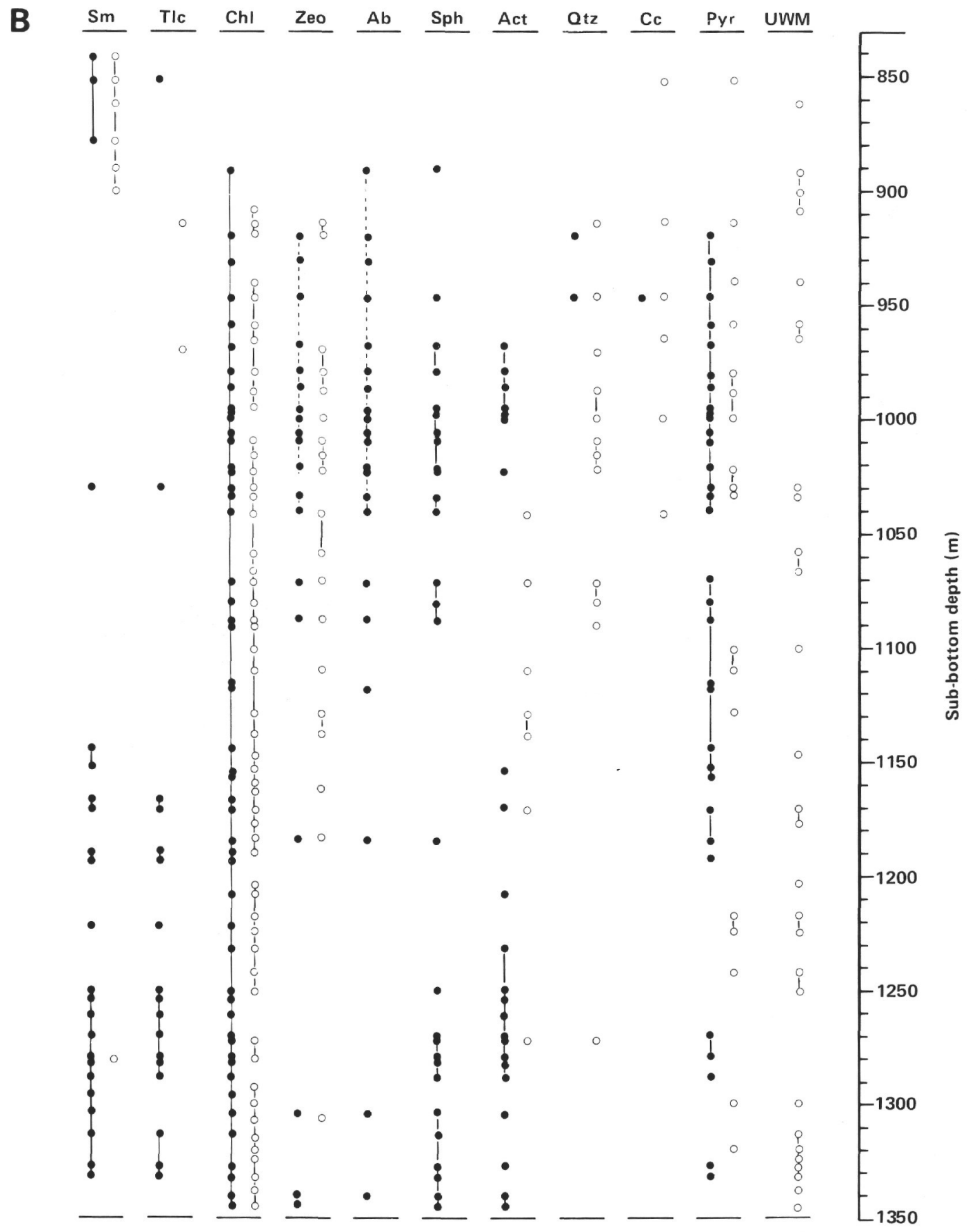

Figure 19. (Continued).

al., 1976). Although the depletion of these phases in magmaphile elements and enrichment in refractory elements is indicative of a more refractory source, preliminary chemical modeling and the $\mathrm{Cr}$ contents of the spinels indicate that the mantle source mineralogy for the CRRZ is that of a spinel lherzolite.

Characteristic differences in clinopyroxene mineral chemistry indicate that parental melts for Groups $\mathbf{M}^{\prime}$, $\mathrm{T}$, and the two LREE-enriched $\mathrm{D}^{\prime}$ (Samples 504B-90-4, $49-53 \mathrm{~cm}$ and 504B-141-1, 16-20 cm) may be generated in portions of the mantle distinct from the source of
Groups D and D'. Clinopyroxene "others" components for these groups exhibit an early enrichment in NaM2, followed by an increase in AlIV at the expense of NaM2. This in turn is followed by an increase in $\mathrm{Ti}$ at the expense of AlIV (Fig. 4B). This trend is not present in most Group $\mathrm{D}^{\prime}$ basalts. It is, however, similar to that seen in clinopyroxenes from alkalic basalts, where it has been attributed to crystallization at progressively shallower depths (Wass, 1979; Kempton, unpublished data). Thus, Groups $\mathbf{M}^{\prime}$, T, and the LREE-enriched Group D' parental melts may have originated at greater depths, or 


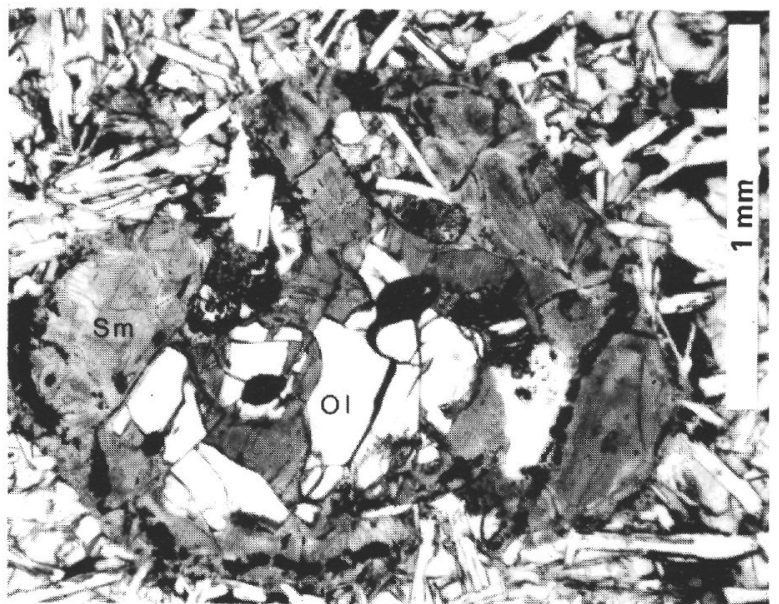

A

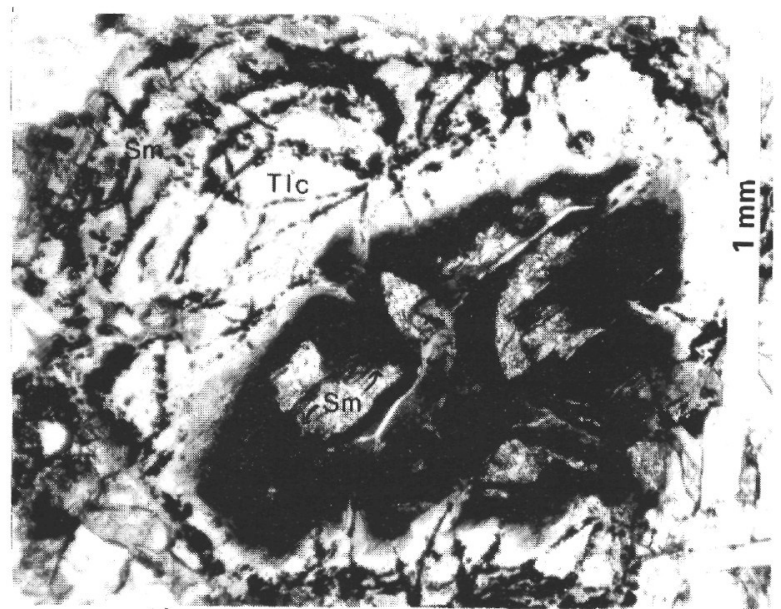

C

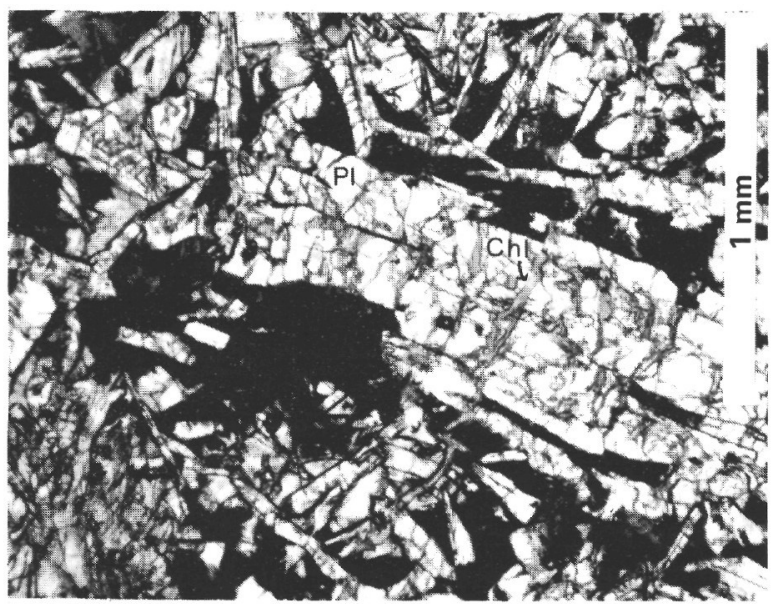

$\mathbf{E}$

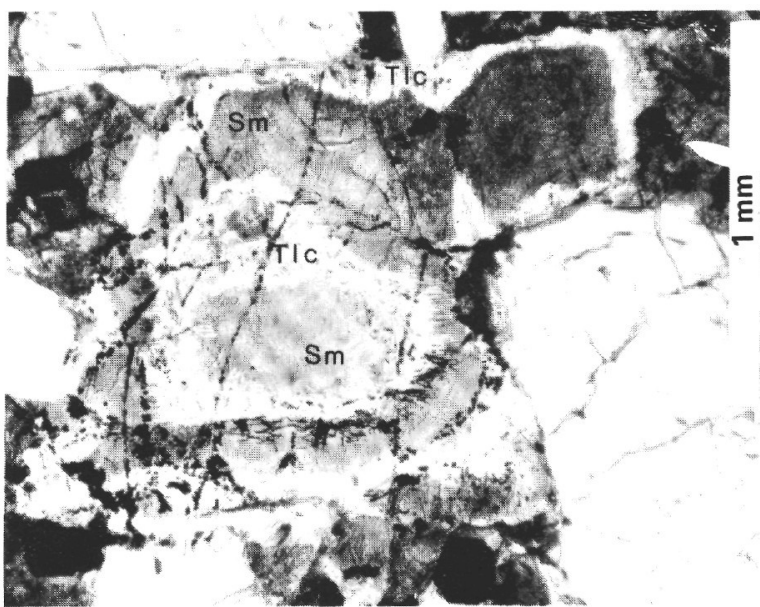

B

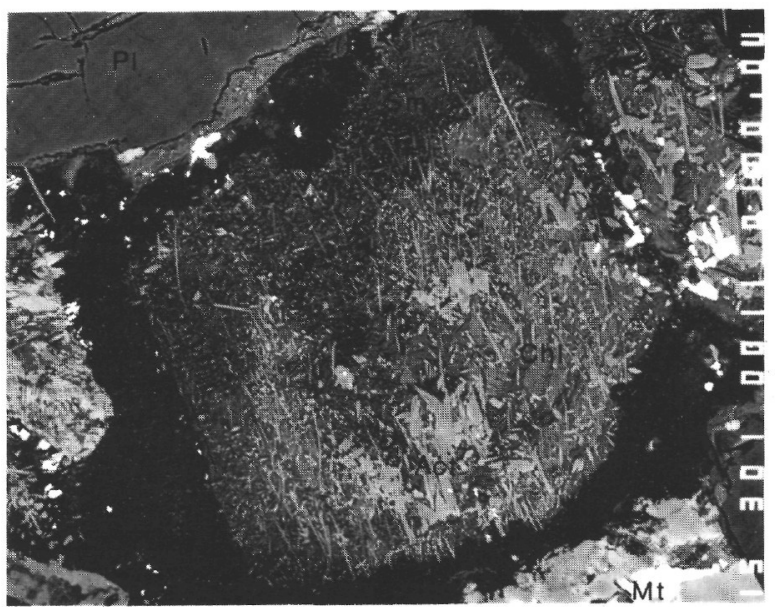

D

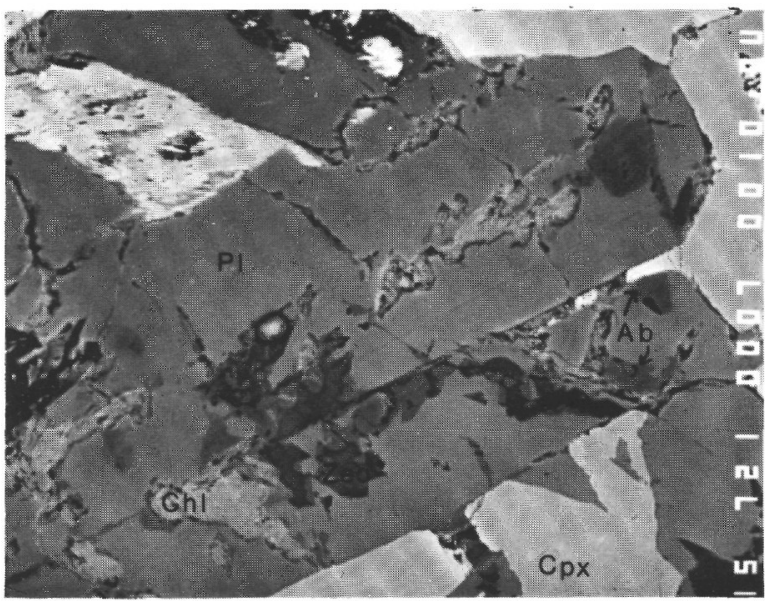

$\mathbf{F}$

Figure 20. (A) Photomicrograph of olivine partially altered to talc, smectite, and magnetite. $\mathrm{Sm}=$ smectite, Ol $=$ olivine. Sample 504B-73-1, 126-130 cm. Uncrossed polars. (B) Photomicrograph of olivine pseudomorph. Notice concentric arrangement of talc and smectite alteration. Smectite has formed after talc. Sample 504B-137-1, 11-16 cm. Uncrossed polars. (C) Photomicrograph of olivine pseudomorph exhibiting concentric arrangement of secondary phases. The dark brown smectite in the core formed directly from olivine. The pale brown smectite formed after talc. (The white areas in the smectite core result from plucking during polishing. Sample 504B-137-1, 11-16 cm. Uncrossed polars. (D) BEI photo of small, groundmass olivine pseudomorph. Secondary minerals include (in increasing BEI intensity): talc (Tlc), smectite (Sm), chlorite (Chl), and actinolite (Act). $\mathrm{Pl}=$ plagioclase and $\mathrm{Cpx}$ $=$ clinopyroxene. Sample 504B-137-1, 11-16 cm. (E) Photomicrograph of alteration of plagioclase to chlorite along fractures. Interstitial areas are altered to chlorite + actinolite. Sample 504B-90-4, 49-53 cm. Uncrossed polars. (F) BEI photo of plagioclase alteration to chlorite along fractures (Chl), and patchy replacement by albite (Ab) and a zeolite (Zeo), epistilbite. Sample 504B$90-4,49-53 \mathrm{~cm}$. 


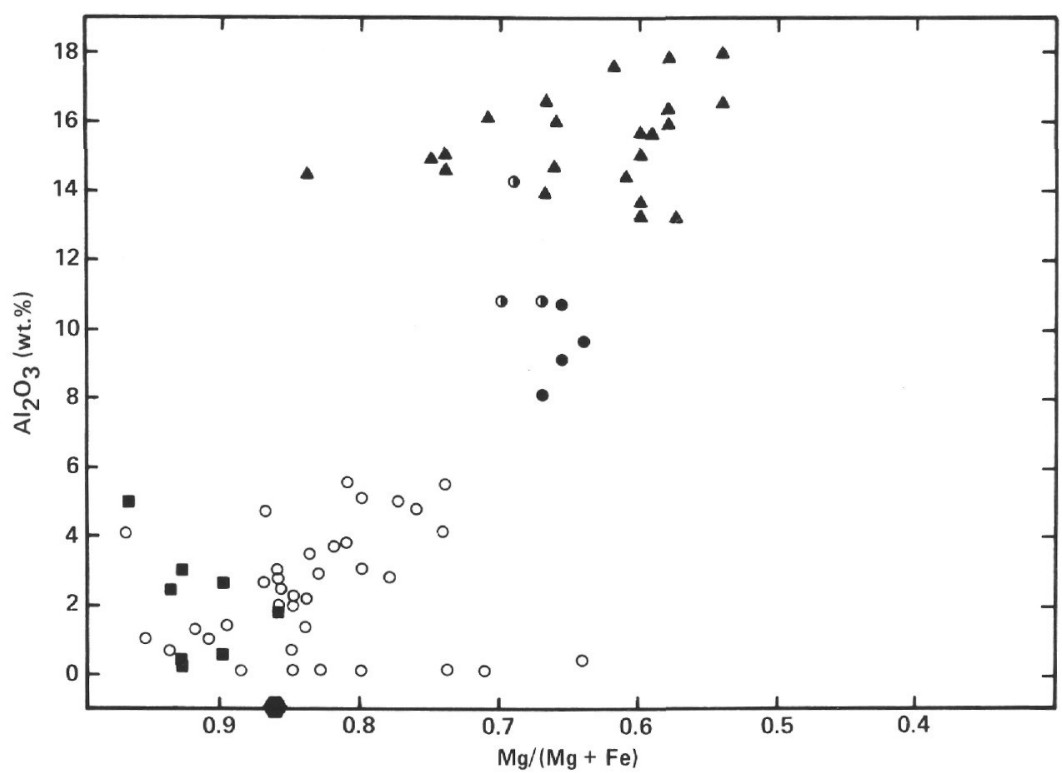

Figure 21. Wt. $\% \mathrm{Al}_{2} \mathrm{O}_{3}$ vs. $\mathrm{Mg} /(\mathrm{Mg}+\mathrm{Fe})$ in secondary phyllosilicates from Hole $504 \mathrm{~B}$, Leg 83. Symbols: $\boldsymbol{\Lambda}$, chlorite; $\boldsymbol{O}$, interstitial smectite; $\boldsymbol{O}$, smectite formed directly from olivine; $O$, smectite formed from talc; $\boldsymbol{\square}$, talc;, olivine analyzed in Sample 504B-73-1, 126-130 cm. Smectites plotting with less than 0.5 wt. $\% \mathrm{Al}_{2} \mathrm{O}_{3}$ are from Sample 504B-73-1, 126-130 cm, in which plagioclase is unaltered. Identification of talc vs. smectite is based on optical properties, percent silica greater than $60 \%$ in talc, and percent $\mathrm{H}_{2} \mathrm{O}$ less than $6.5 \%$.

Table 9. Microprobe analyses of alteration minerals, Leg 83.

\begin{tabular}{lrrrrrrrr}
\hline & $(1)$ & $(2)$ & \multicolumn{1}{c}{$(3)$} & \multicolumn{1}{c}{$(4)$} & \multicolumn{1}{c}{$(5)$} & \multicolumn{1}{c}{$(6)$} & \multicolumn{1}{c}{$(7)$} & \multicolumn{1}{c}{$(8)$} \\
& $83-63^{\mathrm{a}}$ & $83-63$ & $83-63$ & $83-63$ & $83-4$ & \multicolumn{1}{c}{$83-6$} & $83-6$ & $83-18$ \\
\hline $\mathrm{SiO}_{2}$ & 42.62 & 60.45 & 52.49 & 40.58 & 52.45 & 62.81 & 30.45 & 57.53 \\
$\mathrm{TiO}_{2}$ & 0.04 & 0.00 & 0.00 & 0.04 & 0.00 & 0.00 & 0.01 & 0.01 \\
$\mathrm{Al}_{2} \mathrm{O}_{3}$ & 9.71 & 3.07 & 5.61 & 10.84 & 0.10 & 0.31 & 15.12 & 18.33 \\
$\mathrm{FeO}$ & 15.66 & 3.59 & 9.55 & 16.58 & 8.30 & 3.89 & 22.70 & 0.12 \\
$\mathrm{MnO}$ & 0.08 & 0.05 & 0.00 & 0.07 & 0.10 & 0.06 & 0.15 & - \\
$\mathrm{MgO}$ & 15.71 & 25.75 & 22.29 & 18.88 & 25.79 & 27.96 & 18.38 & 0.01 \\
$\mathrm{CaO}$ & 2.16 & 0.01 & 1.13 & 2.24 & 0.64 & 0.07 & 0.16 & 9.16 \\
$\mathrm{NiO}$ & 0.41 & 0.14 & 0.18 & 0.20 & 0.24 & 0.29 & 0.04 & - \\
$\mathrm{Na} 2 \mathrm{O}$ & 0.08 & 0.06 & 0.06 & 0.13 & 0.09 & 0.07 & 0.04 & 0.31 \\
$\mathrm{~K}_{2} \mathrm{O}$ & 0.30 & 0.04 & 0.04 & 0.18 & 0.08 & 0.04 & 0.03 & 0.03 \\
Total & 86.77 & 95.16 & 91.34 & 89.72 & 87.79 & 95.50 & 87.09 & 85.50
\end{tabular}

Cations calculated on the basis of 22 oxygens for $1-6,28(0)$ for $7,16(0)$ for 8

$\begin{array}{lrrrrrrrc}\mathrm{Si} & 6.601 & 7.777 & 7.326 & 6.142 & 7.611 & 8.057 & 6.334 & 5.835 \\ \mathrm{Ti} & 0.004 & 0.000 & 0.000 & 0.004 & 0.000 & 0.000 & 0.000 & 0.001 \\ \mathrm{Al} & 1.768 & 0.464 & 0.921 & 1.932 & 0.017 & 0.045 & 3.708 & 2.191 \\ \mathrm{Fe} & 2.028 & 0.385 & 1.114 & 2.096 & 0.005 & 0.414 & 3.952 & 0.010 \\ \mathrm{Mn} & 0.009 & 0.003 & 0.000 & 0.009 & 0.008 & 0.003 & 0.024 & - \\ \mathrm{Mg} & 3.628 & 5.319 & 4.636 & 4.254 & 5.580 & 5.346 & 5.699 & 0.001 \\ \mathrm{Ca} & 0.354 & 0.000 & 0.167 & 0.359 & 0.098 & 0.007 & 0.030 & 0.995 \\ \mathrm{Ni} & 0.050 & 0.011 & 0.016 & 0.022 & 0.025 & 0.026 & 0.006 & - \\ \mathrm{Na} & 0.023 & 0.015 & 0.012 & 0.035 & 0.021 & 0.015 & 0.012 & 0.061 \\ \mathrm{~K} & 0.059 & 0.003 & 0.004 & 0.031 & 0.013 & 0.003 & 0.006 & 0.003 \\ \mathrm{Total} & 14.524 & 13.979 & 14.197 & 14.883 & 14.377 & 13.917 & 19.771 & 9.097 \\ \mathrm{Mg} / \mathrm{Mg}+\mathrm{Fe} & & & & & & & & \end{array}$

Note: Numbers that follow are core-section, centimeter ranges of samples from Hole 504B. (1) 137-1,

11-16 cm: saponite formed directly from olivine. (2) $137-1,11-16 \mathrm{~cm}$ : talc formed from olivine.

(3) 137-1, 11-16 cm; smectite formed from talc. (4) 137-1, 11-16 cm: interstitial smectite. (5)

$73-1,126-130 \mathrm{~cm}$ : low Al smectite formed from talc in relatively unaltered basalt. (6) 113-1, 129-

$133 \mathrm{~cm}$ : talc formed from olivine. (7) $113-1,129-133 \mathrm{~cm}$ : interstitial chlorite. (8) 90-94, 49-53

$\mathrm{cm}$ : epistilbite replacing plagioclase.

a Sample identification number. 


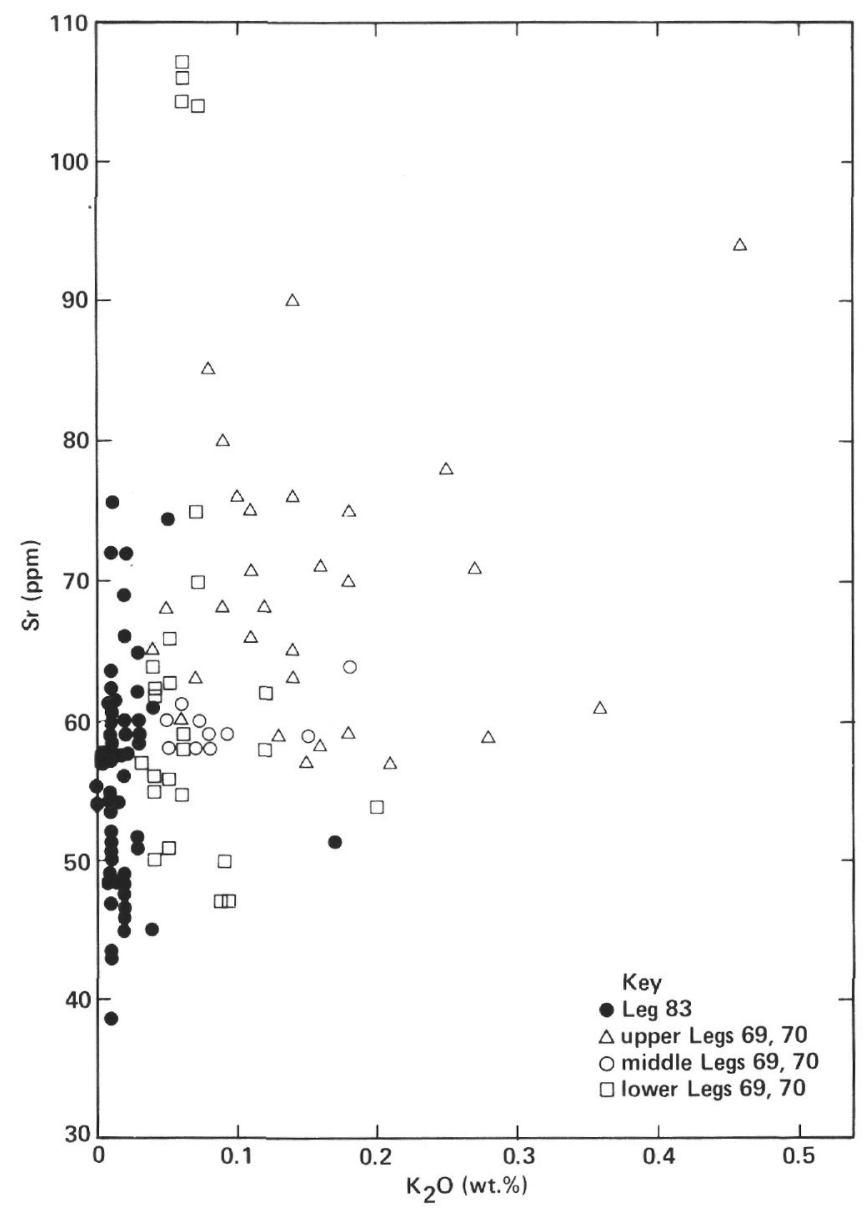

Figure 22. Sr vs. $\mathrm{K}_{2} \mathrm{O}$. Leg 69 and 70 samples are divided on the basis of $\mathrm{Sr}$ and $\mathrm{O}$ isotope information (see text; Barrett and Friedrichsen, 1982). There is a marked decrease in $\mathrm{K}_{2} \mathrm{O}$ and a slight decrease in $\mathrm{Sr}$ with depth, indicating a lessening degree of alteration with depth.

begun crystallizing at greater depths than other basalts from Hole 504B (Irving, 1980; Elthon et al., 1982; Natland et al., 1983).

Based on spinel compositions from Mid-Atlantic Ridge basalts, Sigurdsson and Schilling (1976) proposed that two spiriel fields, magnesiochromites and chromian spinels, resulted from crystallization under different pressure regimes. The low $\mathrm{Cr} /(\mathrm{Cr}+\mathrm{Al})$ aluminous spinels are presumed to be of higher pressure origin than the higher $\mathrm{Cr} /(\mathrm{Cr}+\mathrm{Al})$ magnesiochromites. Natland et al. (1983) adopted this hypothesis for spinels from the upper portion of Hole 504B and suggested that the aluminous spinels were injected into the magma chamber beneath the Costa Rica Rift along with the high-Ca plagioclase and high-Cr clinopyroxene megacrysts. Subsequently, higher $\mathrm{Cr}$, lower $\mathrm{Mg}$ spinels formed at lower pressure in the magma chamber. However, no clear experimental data are available (Dickey and Yoder, 1972; Huebner et al., 1976; Fujii, 1977) to substantiate that such compositional changes occur as a function of pressure.

Furthermore, compositional zonation in several subhedral spinels attached to olivine pseudomorphs in dike margin Sample 504B-111-1, 84-86 cm (Fig. 24) encompasses the entire range of observed $\mathrm{Cr} /(\mathrm{Cr}+\mathrm{Al})(0.478-$ 0.268 ). More importantly, the trend from core to rim is one of decreasing $\mathrm{Cr} /(\mathrm{Cr}+\mathrm{Al})$ and increasing $\mathrm{Mg} /(\mathrm{Mg}$ $+\mathrm{Fe}^{2+}$ ). $\mathrm{MgO}$ increases from 16.8 (core) to $19.3 \mathrm{wt} . \%$ (rim) (Fig. 10 and Table 4). This zonation is opposite of that expected for the crystallization sequence proposed by Natland et al. (1983) and difficult to produce if pressure is the principal controlling factor on composition. Experimental studies by Hill and Roeder (1976) and by Fisk and Bence (1979) find that the composition of spinel is highly sensitive to temperature and oxygen fugacity. High $\mathrm{Cr} /(\mathrm{Cr}+\mathrm{Al})$ magnesiochromite is the liquidus phase between $1230-1250^{\circ} \mathrm{C}$ and $\log \mathrm{f}_{02}=-9$ in FAMOUS basalt glass 527-1-1 (Fisk and Bence, 1979). At lower temperatures $\left(1200^{\circ} \mathrm{C}\right)$ and $\log \mathrm{f}_{02}=-8.5$, an intermediate $\mathrm{Cr}$, higher $\mathrm{Al}$-spinel co-crystallized with $\mathrm{Fo}_{86}$ olivine. These experiments, however, did not synthesize spinels analogous in composition to the low-Cr, high-Al chromian spinels observed in Leg 83 basalts.

The mineralogy of the co-crystallizing phases can also provide a significant control on the spinel composition (Furuta and Tokuyama, 1983). Crystallization of plagioclase competes for $\mathrm{Al}$ and results in a higher $\mathrm{Cr}$ / $(\mathrm{Cr}+\mathrm{Al})$ ratio in spinel, whereas crystallization of olivine competes for $\mathrm{Mg}$ and results in lower spinel $\mathrm{Mg}$ / $(\mathrm{Mg}+\mathrm{Fe})$ values. In Hole 504B, those spinels enclosed in plagioclase or olivine record crystallization in competition with plagioclase, the $\mathrm{Cr} /(\mathrm{Cr}+\mathrm{Al})$ ratio increasing principally by loss of $\mathrm{Al}$ to plagioclase. Crystallization of those spinels found in chilled margins would have ceased prior to extensive plagioclase crystallization so that competition for $\mathrm{Al}$ was not significant. Thus, $\mathrm{Fe}^{3+}$ and $\mathrm{Al}$ increase in these spinels with decreasing temperature, while $\mathrm{Cr}$ is depleted slightly by crystallization of the spinel itself.

Petrographic analysis of intrusive chilled margins from Hole 504B documents the common incorporation of wall rock material into dikes (Kempton, this volume). Partially resorbed plagioclase plus clinopyroxene aggregates present in these basalts have chemical compositions and subophitic textures similar to some massive units and dikes. Such xenoliths could originate by plucking of crystals from the conduit walls as magmas ascend to the surface. Alternatively, the xenoliths may be fragments of cumulates that fractionated from earlier magmas in the magma chamber and were accidentally incorporated upon eruption. Analogous to phenocryst accumulation, addition of such xenoliths and xenocrysts is one mechanism for changing some elements slightly while leaving others constant (Fig. 23). Discrete xenocrysts, however, are also consistent with an origin by magma mixing. Periodic mixing of magmas from small, isolated chambers or repeated injection of magmas into a fractionating magma chamber are possible alternatives. The variations in plagioclase phenocryst and xenocryst composition indicate that a single mixing event is inadequate to explain the data (Figs. 9A-E). Yet, wholerock compositional data are inconsistent with several times repeated magma mixing events. We, therefore, believe that xenocrysts and xenoliths in Hole 504B basalts 

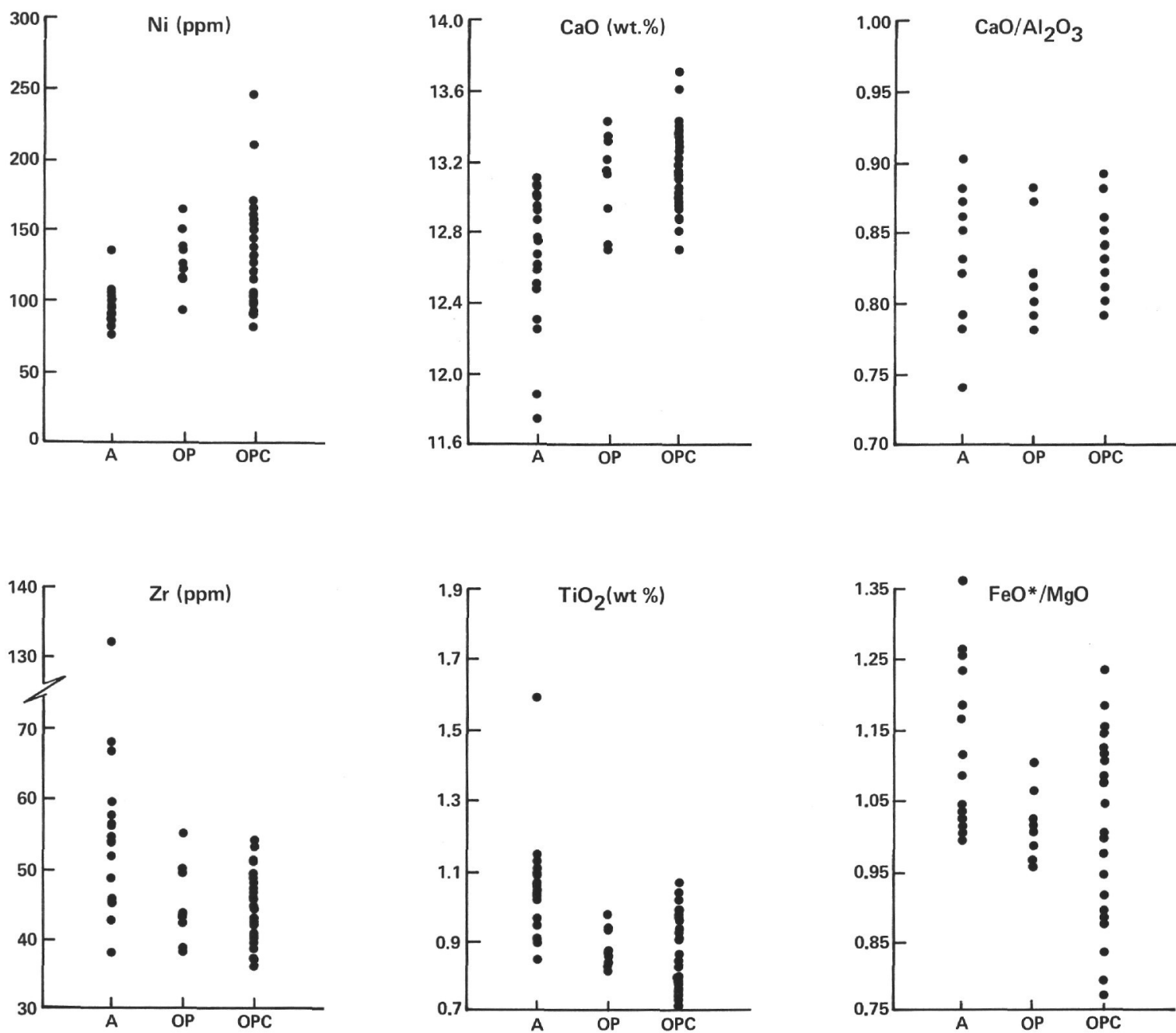

Figure 23. Range in $\mathrm{CaO} / \mathrm{Al}_{2} \mathrm{O}_{3}$ and $\mathrm{FeO} / \mathrm{MgO}$ ratios and $\mathrm{Ni}, \mathrm{CaO}, \mathrm{TiO}_{2}$, and $\mathrm{Zr}$ concentrations with respect to petrographic group. $\mathrm{A}=$ aphyric basalt; $\mathrm{OP}=$ olivine + plagioclase phyric basalt; $\mathrm{OPC}=$ olivine + plagioclase + clinopyroxene phyric basalt. These trends demonstrate that some of the compositional variation in Hole 504B basalts is a consequence of the petrographic type. OP and OPC phyric basalts tend to be higher than aphyric basalts in those compatible elements that would be increased by phenocryst or xenocryst accumulation ( $\mathrm{Ni}, \mathrm{Ca}, \mathrm{Cr}$, etc.); magmaphile elements ( $\mathrm{Ti}, \mathrm{Zr}, \mathrm{Na}$, etc.) are diluted. Ratios of major elements tend to be more constant $\left(\mathrm{CaO} / \mathrm{Al}_{2} \mathrm{O}_{3}\right)$, but analogous variations are apparent $(\mathrm{FeO} *$ / $\mathrm{MgO}$ ).

are not disequilibrium phenocrysts or glomerocrysts produced by magma mixing, but are fragments of crystallized basalt plucked from conduit or magma chamber walls.

\section{CONCLUSIONS}

1. Basalts from Hole 504B (Leg 83) occur as three distinct chemical groups. The majority of the basalts (Group $\mathrm{D}^{\prime}$ ) are extremely depleted in all magmaphile elements, particularly the incompatible ones. No significant differences exist between Groups D (upper portion of Hole 504B; Autio and Rhodes, 1983) and $\mathrm{D}^{\prime}$ (lower portion of Hole 504B) for most trace elements, $\mathrm{TiO}_{2}$, $\mathrm{Fe}_{2} \mathrm{O}_{3}, \mathrm{MgO}$. Groups $\mathrm{M}^{\prime}$ and $\mathrm{T}$ occur only rarely. Group $\mathrm{T}$ is strongly depleted in all magmaphile elements $(\mathrm{Zr}$, $\mathrm{Ti}$, and $\mathrm{Y}$, etc.) except the highly incompatible ones $(\mathrm{Nb}$, $\mathrm{La}$, etc.). Group $\mathrm{M}^{\prime}$ has moderate concentrations of both moderately and highly incompatible elements; it is similar to Group $\mathbf{M}$ from the upper portion of Hole 504B and to Type I MORB (Bryan et al., 1976).
2. Significant differences in magmaphile element abundances and ratios, coupled with similar mg values and compatible element concentrations, indicate that the different chemical groups present at the CRRZ are not comagmatic. Although Groups $D^{\prime}$ and D seem to form a single group related by crystal fractionation, the scatter observed within these groups is too large to be accounted for by fractional crystallization, magma mixing, alteration, or phenocryst addition. The observed differences in magmaphile element ratios $(\mathrm{La} / \mathrm{Sm}, \mathrm{Zr} / \mathrm{Y})$ and abundances can be attributed to mantle source heterogeneity or to multistage melting processes.

3. A decrease in $\mathrm{Sr}$ and $\mathrm{K}_{2} \mathrm{O}$ with depth over the entire length of Hole 504B (Legs 69, 70, and 83) suggests a decreasing interaction between basalt and seawater with depth. The restricted range of $\mathrm{K}_{2} \mathrm{O}$ concentrations in Leg 83 basalts indicates that interaction with seawater is minimal.

4. In general, secondary mineral assemblages indicate that higher temperatures were attained during alter- 


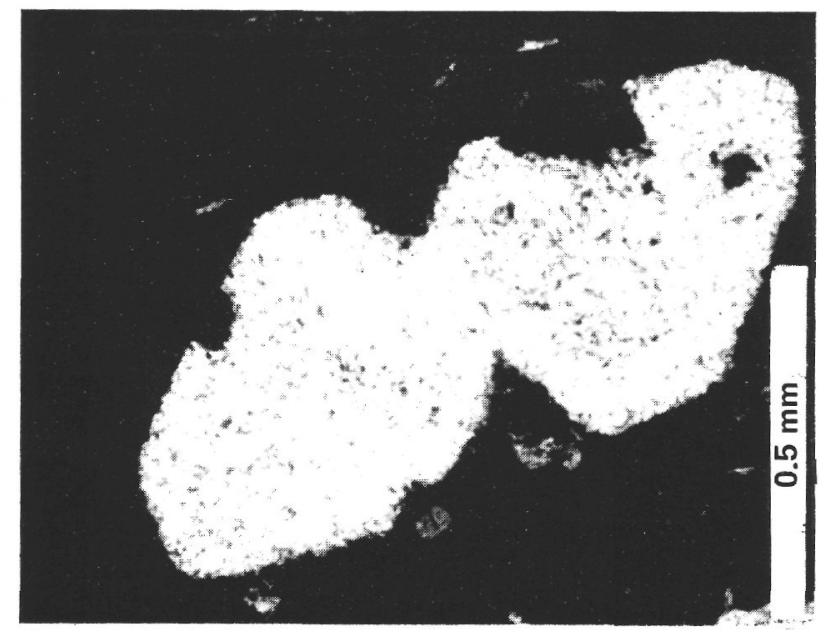

A

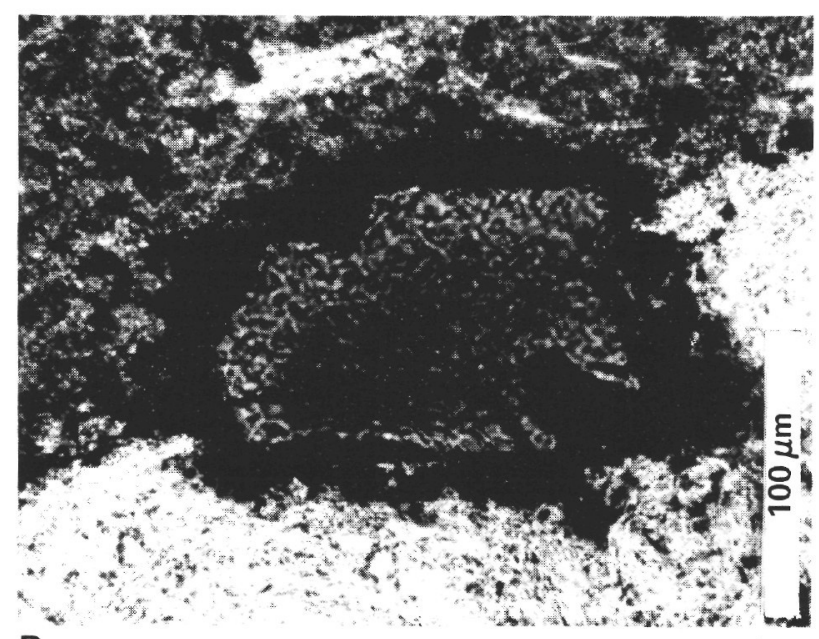

B

Figure 24. Zoned spinel crystals associated with olivine pseudomorphs in dike margin Sample 504B-111-1, 84-86 cm. Dark cores are high in $\mathrm{Cr}$, low in $\mathrm{Al}$ (magnesiochromite); light rims are high in $\mathrm{Al}$, low in $\mathrm{Cr}$ (chromian spinel). Uncrossed polars. (A) Width of field is $1.3 \mathrm{~mm}$. (B) Width of field is $0.3 \mathrm{~mm}$.

ation in the lower portion of Hole 504B (Leg 83) relative to the upper portion (Legs 69 and 70). However, a systematic progression of alteration with depth for the interval below $836 \mathrm{~m}$ BSF (Leg 83) is not apparent. Rather, the degree of alteration appears to be a function of the initial texture and fracture density. Variations in wholerock major and trace element concentrations cannot be attributed to differences in alteration observed. Variations in secondary mineral compositions indicate that in most cases extensive remobilization of elements has not occurred; the major and trace element signature of these basalts remains similar to those of the original composition.

5. Xenolithic aggregates of clinopyroxene + plagioclase are present in basalts from Hole 504B. Similarities in texture and mineral composition between these xenoliths and many medium-grained basalts in the lower portion of the hole suggest that incorporation of solid com- ponents, either from conduit or magma chamber walls, is a major source of disequilibrium crystals and provides small enrichments in such elements as $\mathrm{Ca}, \mathrm{Mg}, \mathrm{Ni}$, $\mathrm{Cr}$, etc.

6. Xenocrystic clinopyroxenes exhibit characteristically high concentrations of $\mathrm{Mg}, \mathrm{Ca}$, and $\mathrm{Cr}$ relative to coexisting phenocryst and groundmass clinopyroxenes. Xenocrysts in Groups $\mathrm{M}^{\prime}, \mathrm{T}$, and two $\mathrm{D}^{\prime}$ basalts also exhibit distinctive zonation in clinopyroxene "others" components, being first enriched in NaM2, followed by enrichment in AlIV, followed by enrichment in Ti (principally by diminishing concentrations of $\mathrm{Al}$ ). These trends are similar to those seen in alkalic basalts and have been attributed to crystallization under decreasing pressure conditions (Wass, 1979). Rarely occurring Groups $\mathbf{M}^{\prime}$ and $T$ appear to be generated in portions of the mantle distinct from the source for Group $\mathrm{D}^{\prime}$, possibly at greater depth, but Group $\mathrm{M}^{\prime}$ could also be generated by complex melting in the same source region as Group $\mathrm{D}^{\prime}$.

7. Hole 504B plagioclase compositions are distinctive in their relatively high anorthite content, up to $90 \mathrm{~mol} \%$. Plagioclase xenocrysts occur both more and less calcic than plagioclase phenocrysts, but are always lower in $\mathrm{Mg} /(\mathrm{Mg}+\mathrm{Fe})$. The spectrum of zoning trends oberved within and among samples indicates that a single mixing event is inadequate to explain the composition variation in plagioclase phenocrysts and xenocrysts in basalts from Hole 504B.

8. Hole 504B is the first reference section of oceanic basement greater than $600 \mathrm{~m}$ in length $(1075.5 \mathrm{~m})$ (Anderson et al., 1982) and includes the first recovered samples from the sheeted dike complex of Layer $2 \mathrm{C}$ in the oceanic crust. However, compared to other mid-ocean ridge basalts, the CRRZ basalts are unusual in composition. They are low in incompatible element abundances but are not primitive, having undergone substantial magmatic evolution. If our conclusions are correct, that is, that most basalts from Hole 504B come from a relatively depleted mantle, then one might expect the thickness of crustal layers in the Costa Rica Rift Zone to be less than those of typical oceanic crust. This model could account for the intersection of the sheeted dikes (Layer 2C) at relatively shallow depths in Hole 504B.

\section{ACKNOWLEDGMENTS}

This work has been partially supported by NSF Grant EAR 8026451-01 to M. A. Dungan; NSF Grant OCE 82-15632 to J. M. Rhodes; and funds from the Institute for the Study of Earth and Man, SMU, to P. D. Kempton.

We wish to thank J. Sparks for technical assistance at the University of Massachusetts and D. Blanchard for use of the INAA facilities at NASA-JSC, Houston. The authors also wish to thank J. Natland and the anonymous reviewer for their careful and insightful comments.

\section{REFERENCES}

Anderson, R. N., Honnorez, J., Becker, K., Adamson, A. C., Emmerman, R., Kempton, P. D., Kinoshita, H., Laverne, C., Mottl, M. J., and Newmark, R. L., 1982. DSDP Hole 504B, the first reference section over $1 \mathrm{~km}$ through Layer 2 of the ocean crust. $\mathrm{Na}$ ture, 300:589-594.

Autio, L. K., and Rhodes, J. M., 1981. Basalts from Holes 501 and 504B (Costa Rica Rift Zone): A possible source for high anorthite 
phenocrysts in ocean-floor basaltic magmas. EOS (Trans. Am. Geophys. Union), 62:309. (Abstract)

1983. Costa Rica Rift Zone basalts: Geochemical and experimental data from a possible example of multistage melting. In Cann, J. R., Langseth, M. G., Honnorez, J., Von Herzen, R. P., White, S. M., et al., Init. Repts. DSDP, 69: Washington (U.S. Govt. Printing Office), 729-745.

Ayuso, R. A., Bence, A. E., and Taylor, S. R., 1976. Upper Jurassic tholeitic basalts from DSDP Leg 11. J. Geophys. Res., 81: 4305-4325.

Barrett, T. J., and Friedrichsen, H., 1982. Strontium and oxygen isotopic composition of some basalts from Hole 504B, Costa Rica Rift, DSDP Legs 69 and 70. Earth Planet. Sci. Lett., 60:27-38.

Basaltic Volcanism Study Project, 1981. Basaltic Volcanism on the Terrestrial Planets: New York (Pergamon Press, Inc.).

Bass, M. N., Moberly, R., Rhodes, J. M., Shih, C., and Church, S. E., 1975. Volcanic rocks cored in the central Pacific, Leg 17, Deep Sea Drilling Project. In Winterer, E. L., Ewing, J. I., et al., Init. Repts. DSDP, 17: Washington (U.S. Govt. Printing Office), 429-446.

Bence, A. E., and Albee, A. L., 1968. Empirical correction factors for the electron microanalysis of silicates and oxides. J. Geol., 76: 382-403.

Bernas, B., 1968. A new method for decomposition and comprehensive analysis of silicates of atomic absorption spectrometry. Anal. Chem., 40:1682-1686.

Bougault, H., Cambon, P., Corre, O., Joron, J. L., and Treuil, M., 1979. Evidence for variability of magmatic processes and upper mantle heterogeneity in the axial region of the Mid-Atlantic Ridge near $22^{\circ}$ and $36^{\circ} \mathrm{N}$. Tectonophysics, 55:11-34.

Bryan, W. B., Thompson, G., Frey, F. A., and Dickey, J. S., 1976. Inferred settings and differentiation in basalts from the Deep Sea Drilling Project. J. Geophys. Res., 81:4285-4304.

Clague, D. A., Frey, F. A., Thompson, G., and Rindge, S., 1981. Minor and trace element geochemistry of volcanic rocks dredged from the Galapagos Spreading Center: Role of crystal fractionation and mantle heterogeneity. J. Geophys. Res., 86:9469-9482.

Dick, H. J. B., and Bullen, T., in press. Chromian spinel as a petrogenetic indicator in oceanic environments. Contrib. Mineral. Petrol.

Dickey, J. S., and Yoder, H. S., 1972. Partitioning of chromium and aluminum between clinopyroxene and spinel. Carnegie Inst. Washington Yearbook, 71:384-392.

Drake, M. J., 1976. Plagioclas-melt equilibria. Geochim. Cosmochim. Acta, 40:457-466.

Duncan, R. A., and Green, D. H., 1980. Role of multistage melting in the formation of oceanic crust. Geology, 8:22-26.

Dungan, M. A., and Rhodes, J. M., 1978. Residual glasses and melt inclusions in basalts from DSDP Legs 45 and 46: Evidence for magma mixing. Contrib. Mineral. Petrol., 67:417-431.

Elthon, D., Casey, J., and Komar, S., 1982. Mineral chemistry of ultramafic cumulates from the North Arm Mountain Massif of the Bay of Islands ophiolite: Evidence for high-pressure crystal fractionation of oceanic basalts. EOS (Trans. Am. Geophys. Union), 63:475. (Abstract)

Erlank, A. J., and Kable, E. J. D., 1976. The significance of incompatible elements in Mid-Atlantic Ridge basalts from $45^{\circ} \mathrm{N}$ with particular reference to $\mathrm{Zr} / \mathrm{Nb}$. Contrib. Mineral. Petrol., 54:281-291.

Etoubleau, J., Corre, O., Joron, J. L., Bougault, H., and Treuil, M., 1983. Costa Rica Rift: Variably deleted basalts in the same hole. In Cann, J. R., Langseth, M. G., Honnorez, J., Von Herzen, R. P., White, S. M., et al., Init. Repts. DSDP, 69: Washington (U.S. Govt. Printing Office), 765-773.

Fisk, M. R., and Bence, A. E., 1979. Experimental studies of spinel crystallization in FAMOUS basalt 527-1-1. EOS (Trans. Am. Geophys. Union), 60:420. (Abstract)

Frey, F. A., Bryan, W. B., and Thompson, G., 1974. Atlantic Ocean floor; Geochemistry and petrology of basalts from legs 2 and 3 of the Deep Sea Drilling Project. J. Geophys. Res., 79:5507-5527.

Fujii, T., 1977. Fe-Mg partitioning between olivine and spinel. Carnegie. Inst. Washington Yearbook, 76:563-569.

Furuta, T., and Tokuyama, H., 1983. Chromian spinels in Costa Rica Rift basalts, Deep Sea Drilling Project Site 505-A preliminary interpretation of electron microprobe analyses. In Cann, J. R., Langseth, M. G., Honnorez, J., Von Herzen, R. P., White, S. M., et al.,
Init. Repts. DSDP, 69: Washington (U.S. Govt. Printing Office), 805-810.

Haggerty, S. E., 1976a. Opaque mineral oxides in terrestrial igneous rocks. In Rumble, D., III (Ed.), Oxide Minerals, Min. Soc. Am. Short Course Notes: Virginia (Southern Printing Co.), pp. Hg-101-Hg-300.

1976b. Oxidation of opaque mineral oxides in basalts. In Rumble, D., III (Ed.), Oxide Minerals, Min. Soc. Am. Short Course Notes: Virginia (Southern Printing Co.), pp. Hg-1-Hg-100.

Haskin, L. A., Haskin, M. A., Frey, F. A., and Wildeman, T. R., 1968. Relative and absolute terrestrial abundances of the rare earths. In Ahrens, L. H. (Ed.), Origin and distribution of the elements: New York (Pergamon Press), pp. 889-912.

Hill, R., and Roeder, P., 1974. The crystallization of spinel from basaltic liquid as a function of oxygen fugacity. J. Geol., 82:709-729.

Huebner, J. S., Lipin, B. R., and Wiggins, L. B., 1976. Partitioning of chromium between silicate crystals and melts. Proc. Lunar Sci. Conf., 7:1195-1220.

Irving, A. J., 1980. Petrology and geochemistry of composite ultramafic xenoliths in alkali basalts and implications for magmatic processes within the mantle. Am. J. Sci., 280A:389-426.

Kuo, L., and Kirkpatrick, R. J., 1982. Pre-eruption history of phyric basalts from DSDP Legs 45 and 46: Evidence from morphology and zoning patterns in plagioclase. Contrib. Mineral. Petrol., 79: 13-27.

Langmuir, C. H., Bender, J. F., Bence, A. E., and Hanson, G. N., 1977. Petrogenesis of basalts from the FAMOUS area: Mid-Atlantic Ridge. Earth Planet. Sci. Lett., 36:133-156.

Liias, R. A., and Rhodes, J. M., 1982. Does a mantle plume influence basalt composition along the Juan de Fuca Ridge? EOS (Trans. Am. Geophys. Union), 63:1153-1154. (Abstract)

Lofgren, G. E., and Norris, P. N., 1981. Experimental duplication of plagioclase sieve and overgrowth textures. Geol. Soc. Am. Abstracts with Programs, 13(7):498. (Abstract)

Marsh, N. G., Tarney, J., and Hendrey, G. L., 1983. Trace element geochemistry of basalts from Hole 504B, Panama Basin, Deep Sea Drilling Project Legs 69 and 70. In Cann, J. R., Langseth, M. G., Honnorez, J., Von Herzen, R. P., White, S. M., et al., Init. Repts. DSDP, 69: Washington (U.S. Govt. Printing Office), 747-763.

Natland, J. H., Adamson, A. C., Laverne, C., Melson, W. G., and O'Hearn, T., 1983. A compositionally nearly steady state magma chamber at the Costa Rica Rift: Evidence from basalt glass and mineral data Deep Sea Drilling Project Sites 501, 504, and 505. In Cann, J. R., Langseth, M. G., Honnorez, J., Von Herzen, R. P., White, S. M., et al., Init. Repts. DSDP, 69: Washington (U.S. Govt. Printing Office), 811-858.

Norrish, K., and Chappell, B. W., 1967. X-ray fluorescence spectrometry. In Zussman, J. (Ed.), Physical Methods in Determinative Mineralogy: New York (Academic Press), pp. 161-214.

Norrish, K., and Hutton, J. T., 1969. An accurate X-ray spectrographic method for the analysis of a wide range of geological samples. Geochim. Cosmochim. Acta, 33:431-453.

Reynolds, R. C., 1967. Matrix corrections in trace element analysis by $\mathrm{X}$-ray fluorescence: Estimation of the mass absorption coefficient by Compton scattering. Am. Mineralogist, 48:1133-1143.

Rhodes, J. M., Blanchard, D. P., Dungan, M. A., Rodgers, K. V., and Brannon, J. C., 1978. Chemistry of Leg 45 basalts. In Melson, W. G., Rabinowitz, P. D., et al., Init. Repts. DSDP, 45: Washington (U.S. Govt. Printing Office), 447-459.

Rhodes, J. M., Blanchard, D. P., Rodgers, K. V., Jacobs, J. W., and Brannon, J. C., 1976. Petrology and chemistry of basalts from the Nazca Plate: Part 2-Major and trace element chemistry. In Yeats, R. S., Hart, S. R., et al., Init. Repts. DSDP, 34: Washington (U.S. Govt. Printing Office), 239-244.

Roeder, P. L., and Emslie, R. F., 1970. Olivine-liquid equilibrium. Contrib. Mineral. Petrol., 19:175-289.

Schilling, J.-G., 1971. Sea-floor evolution: Rare-earth evidence. Phil. Trans. R. Soc. London, A268:663-706.

Schilling, J.-G., Anderson, R. N., and Vogt, P., 1976. Rare earth, Fe and Ti variations along the Galapagos Spreading Center, and their relationship to the Galapagos mantle plume. Nature, 261:108-113.

Sigurdsson, H., and Schilling, J.-G., 1976. Spinels in Mid-Atlantic Ridge basalts: Chemistry and occurrence. Earth Planet. Sci. Lett., 29:7-20. 
Tarney, J., Saunders, A. D., Weaver, S. D., Donnellen, N. C. B., and Hendry, G. L., 1979. Minor-element geochemistry of basalts from Leg 49, North Atlantic Ocean. In Luyendyk, B. P., Cann, J. R., et al., Init. Repts. DSDP, 49: Washington (U.S. Govt. Printing Office), 657-691.

Wass, S., 1979. Multiple origins of clinopyroxenes in alkali basaltic rocks. Lithos, 12:115-132.
Wood, D. A., 1979. Dynamic partial melting: Its application to the petrogenesis of basalt lava series from Iceland, the Faeroe Islands, the Isle of Skye (Scotland), and the Troodos Massif (Cyprus). Geochim. Cosmochim. Acta, 43:1031-1046.

Date of Initial Receipt: 22 August 1983 Date of Acceptance: 15 January 1984 